\title{
Synthesis of 1-indanones with a broad range of biological activity
}

\author{
Marika Turek ${ }^{1}$, Dorota Szczęsna ${ }^{2,3}$, Marek Koprowski ${ }^{3}$ and Piotr Bałczewski ${ }^{* 1,3}$
}

\author{
Review \\ Address: \\ ${ }^{1}$ Institute of Chemistry, Environmental Protection and Biotechnology, \\ The Faculty of Mathematics and Natural Sciences, Jan Długosz \\ University in Częstochowa, Armii Krajowej 13/15, Częstochowa, \\ 42-201, Poland, ${ }^{2}$ Department of Structural Biology, Faculty of \\ Biomedical Sciences and Postgraduate Education, Medical University \\ of Łódź, Żeligowskiego 7/9, 90-752, Łódź, Poland and ${ }^{3}$ Department of \\ Heteroorganic Chemistry, Centre of Molecular and Macromolecular \\ Studies, Polish Academy of Sciences, Sienkiewicza 112, Łódź, \\ 90-363, Poland \\ Email: \\ Piotr Bałczewski - pbalczew@cbmm.lodz.pl \\ * Corresponding author \\ Keywords: \\ biological activity; Diels-Alder reaction; Friedel-Crafts reaction; \\ 1-indanones; Nazarov reaction
}

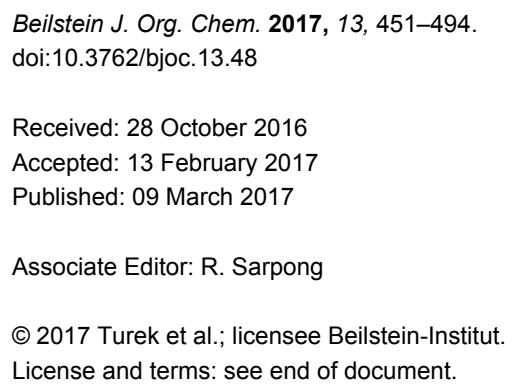

\begin{abstract}
This comprehensive review describes methods for the preparation of 1-indanones published in original and patent literature from 1926 to 2017. More than 100 synthetic methods utilizing carboxylic acids, esters, diesters, acid chlorides, ketones, alkynes, alcohols etc. as starting materials, have been performed. This review also covers the most important studies on the biological activity of 1 -indanones and their derivatives which are potent antiviral, anti-inflammatory, analgesic, antimalarial, antibacterial and anticancer compounds. Moreover, they can be used in the treatment of neurodegenerative diseases and as effective insecticides, fungicides and herbicides.
\end{abstract}

\section{Introduction}

In the last few years, 1-indanone derivatives and their structural analogues have been widely used in medicine, agriculture and in natural products synthesis [1-3]. In addition, structurally related indanes also showed biological activity and have been reviewed by Ahmed in 2016 [4]. Extensive studies on bioactivity of 1-indanone derivatives open up more and more new possibilities of their applications as antiviral and antibacterial agents [5] (I and II), anticancer drugs [6] (VI), pharmaceuticals used in the Alzheimer's disease treatment [7] (III), cardiovascular drugs [7] (IV), insecticides, fungicides, herbicides [8] (V) and non-nucleoside, low molecular drugs for the hepatitis $\mathrm{C}$ treatment, which inhibit HCV replication [9,10] (Figure 1). 
<smiles>[R]c1c([R])c([R])c2c(c1[R])CC/C2=N\NC(N)=S</smiles>

I $\mathrm{R}^{1}-\mathrm{R}^{4}=\mathrm{H}, \mathrm{Me}$<smiles>[R]C1c2cc(C)c(CO)c([N+](=O)[O-])c2C(=O)C1C</smiles>

II $\mathrm{R}=\mathrm{H}$ or $\mathrm{OH}$<smiles>C[N+]([O-])(c1ccccc1)C1Cc2cc(OCC(=O)O)c(Cl)c(Cl)c2C1=O</smiles>

III<smiles>COc1cc2c(cc1OC)C(=O)C(CC1CCC(Br)CC1)C2</smiles><smiles>COC1CC(=O)c2c(C=O)cc(C)cc21</smiles>

VI

$$
\begin{aligned}
& \mathrm{R}^{1}, \mathrm{R}^{2}=\text { alkyl } \\
& \mathrm{R}^{3}=N \text {-piperidinyl- }\left(\mathrm{CH}_{2}\right)_{n^{-}} \\
& n=2,3,5
\end{aligned}
$$

Figure 1: Biologically active 1-indanones and their structural analogues.

First publications concerning the preparation of 1-indanones appeared in the 1920s and since then this field has been intensively developed [11]. A huge interest in 1-indanones and their derivatives resulted in a considerable number of papers concerning their synthesis (Figure 2). The commonly used reaction in this area is the Nazarov reaction which employs $\alpha, \beta$-unsatu-

(a) Number of papers about 1-indanones

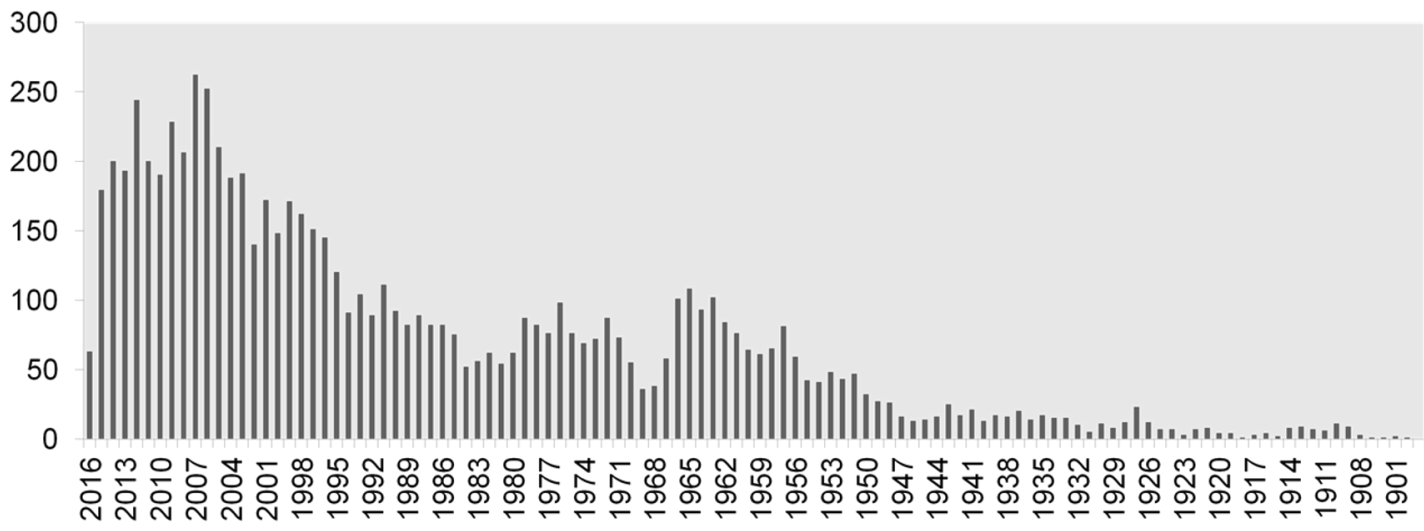

(b) Number of papers about synthesis of 1 -indanones

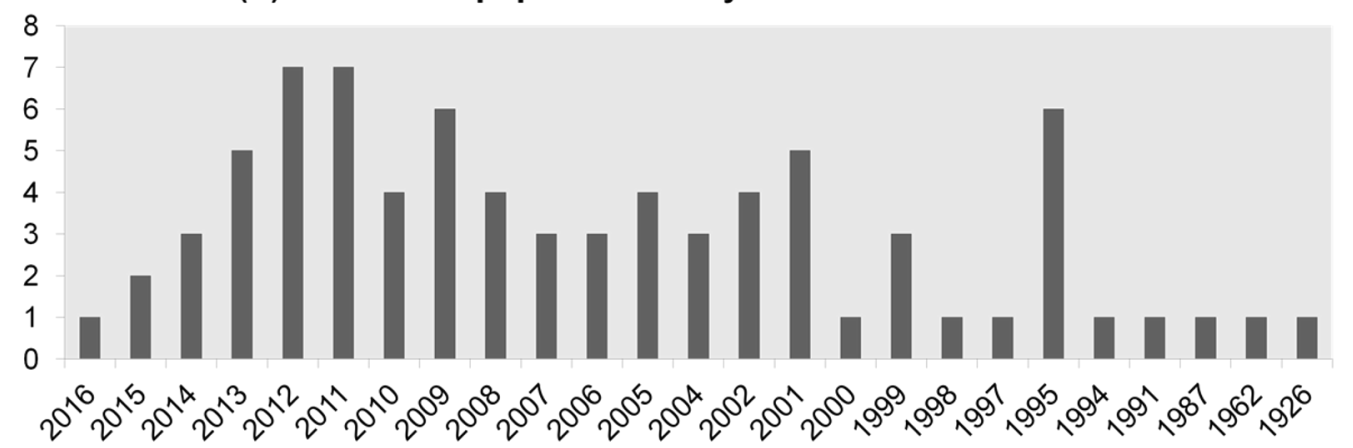

Figure 2: Number of papers about (a) 1-indanones, (b) synthesis of 1-indanones. 
rated ketones as substrates and is carried out in the presence of Brønsted or Lewis acids. Despite extensive studies on 1 -indanones and their biological activity, this group of compounds has never been reviewed in literature and therefore the present work is the first, comprehensive review of synthetic methods and applications of these compounds in medicine and agriculture published from 1926 to 2017.

We have divided the review into 4 sections, taking into account the formation of the 5- (section 1), 6- (section 2) and simultaneous formation of 5-and 6-membered rings of 1-indanones (section 3) as well as functionalization of 1-indanones or related compounds (section 4).

\section{Review}

\section{Construction of the 5-membered ring}

\subsection{From carbonyl compounds}

1.1.1 From carboxylic acids: The first synthesis of 1-indanone from carboxylic acid has been described by Price and Lewis in 1939 [12]. They cyclized hydrocinnamic acid (1) to the unsubstituted 1 -indanone (2), in 27\% yield using 20\% sulfuric acid at $140{ }^{\circ} \mathrm{C}$ (Scheme 1).

$$
\text { OH } \frac{\mathrm{H}_{2} \mathrm{SO}_{4}(20 \%)}{140^{\circ} \mathrm{C}, 5 \mathrm{~min}}
$$

1

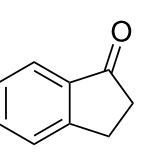

2
Scheme 1: Synthesis of 1-indanone (2) from hydrocinnamic acid (1).

Another reaction leading to the formation of the unsubstituted 1 -indanone (2) in higher $76 \%$ yield utilizes the cyclization of 3-(2-bromophenyl)propionic acid (3), conducted at $-100{ }^{\circ} \mathrm{C}$ in the presence of $n$-BuLi (Scheme 2) [13].

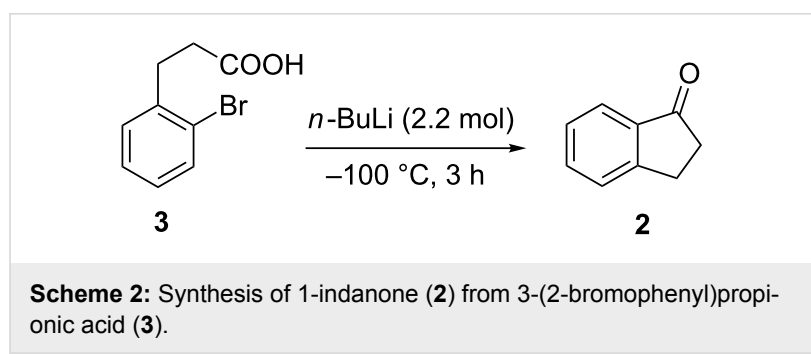

A cyclization of 3 -arylpropionic acids 4 , catalyzed by $\mathrm{Tb}(\mathrm{OTf})_{3}$ at $250{ }^{\circ} \mathrm{C}$, led to the formation of at the aryl ring substituted 1 -indanones 5 in yields of up to $74 \%$ and trace amounts of the auto-condensation products 6 (Scheme 3). Even in the deactivated derivatives containing halogen atoms at the aromatic system, the cyclopentanone ring closure took place quite easily [14]. If other Lewis acids, such as $\mathrm{Bi}\left(\mathrm{NTf}_{2}\right)_{3}$ or triflate derivatives of the transition metals (In, Sc, Ce, Pr, Nd, Eu, Dy, Yb, $\mathrm{Lu}, \mathrm{Hf}, \mathrm{Gd}$ ) were used, the cyclization proceeded in unsatisfactory yields and with a large number of unidentified byproducts.

Cyclization of other 3-arylpropionic acids 7 led to the formation of 4,7-dimethoxy-1-indanones $\mathbf{8}$. They were used in the key step of the synthesis of kinamycin 9 derivatives, which exhibited a strong cytotoxic and anticancer activity (Scheme 4) [15].

Propionic acid derivatives are also useful substrates in syntheses of 1-indanones and isocoumarins [16]. These latter are essential reagents for the synthesis of bioactive compounds. Fluoroorganic compounds play a significant role as very effective therapeutics, and for this reason, Prakash, Olah et al. synthesized in 2010 trifluoromethyl-substituted arylpropanoic acids 12, 1-indanones 13 and dihydrocoumarins 14 (Scheme 5) [17]. These products have been obtained by utilizing arenes/phenols $11(\mathrm{X}=\mathrm{H} / \mathrm{OH})$ and 2-(trifluoromethyl)acrylic acid (10) as a result of a Friedel-Crafts alkylation.

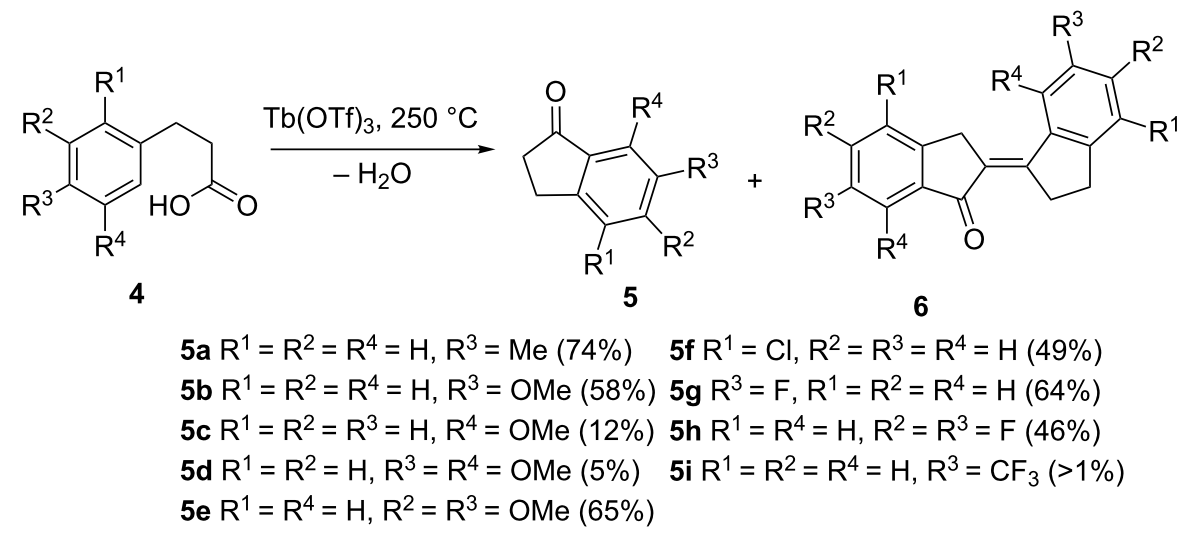




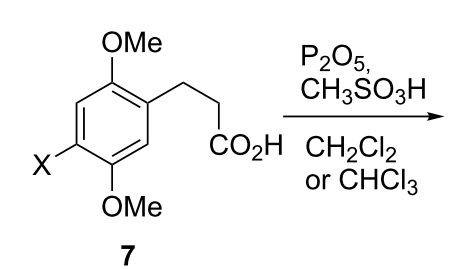

$X=H(67 \%), \operatorname{Br}(71 \%)$<smiles>[X]c1cc(OC)c2c(c1OC)C(=O)CC2</smiles>

8

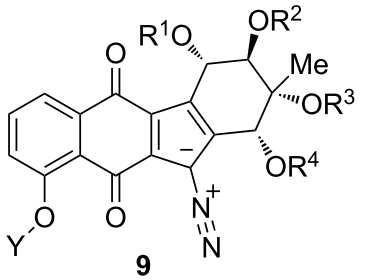

9a kinamycin $\left(R^{1}, R^{2}, R^{3}, R^{4}=H\right.$ or $\left.A c, Y=H\right)$

9b methylkinamycin $C$

$\left(R^{1}=R^{2}=R^{4}=A c, R^{3}=H, Y=M e\right)$

Scheme 4: Synthesis of kinamycin (9a) and methylkinamycin C (9b).

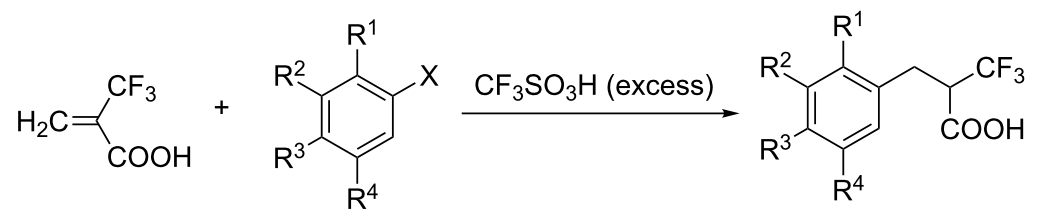

10

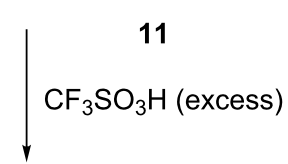<smiles>[R]c1c([R])c([R])c2c(c1[R])CC(C(F)(F)F)C(=O)O2</smiles>

14
12

$\mathrm{CF}_{3} \mathrm{SO}_{3} \mathrm{H}$ (excess)<smiles>[R]c1c([R])c([R])c2c(c1[R])CC(C(F)(F)F)C2=O</smiles>

13

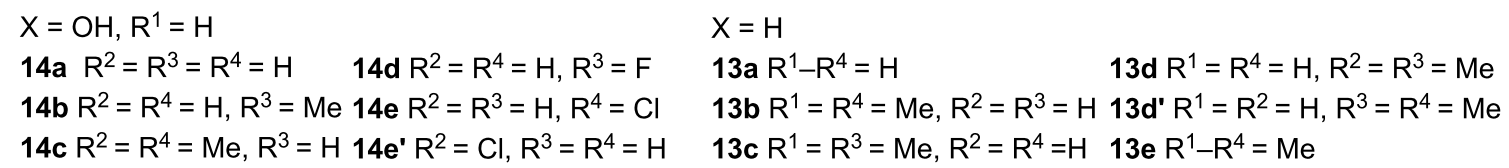

Scheme 5: Synthesis of trifluoromethyl-substituted arylpropionic acids 12, 1-indanones 13 and dihydrocoumarins 14.

An efficient and scalable one-pot process for the preparation of 1-indanones from benzoic acids has been described by Huang et al. [18]. In this synthesis, acyl chlorides formed in the reaction of benzoic acids $\mathbf{1 5}$ with thionyl chloride, reacted with ethylene and the resulting intermediates underwent an intramolecular Friedel-Crafts alkylation to form 1-indanones $\mathbf{1 6}$ (Scheme 6).

Both arylpropionic and 3-arylacrylic acids $\mathbf{1 7}$ underwent cyclization in the presence of polyphosphoric and sulfuric acids to form 1-indanones 18 in good yields (60-90\%) (Scheme 7) [19].

A one-step synthesis of 1 -indanones 22 through the $\mathrm{NbCl}_{5}$-induced Friedel-Crafts reaction, has been described by Barbosa et al. in 2015 [20]. The reaction was carried out using 3,3- dimethylacrylic acid (19), aromatic substrate 20 and highly oxophilic $\mathrm{NbCl}_{5}$ as a catalyst. By varying the type of substrate, a variety of 1-indanone derivatives 22 was obtained. Depending on the reaction conditions $(\mathrm{A}-\mathrm{C}), 1$-indanone derivatives 22 was obtained in $0-78 \%$ yields. The studies indicated that of two possible intermediates $21 \mathbf{a}$ and $\mathbf{2 1 b}$, obtained as a result of acylation or alkylation reaction, the intermediate 21a with activated aromatic ring, always led to the 1-indanone formation, in contrast to the acylated intermediate $\mathbf{2 1 b}$ with deactivated aromatic ring (Scheme 8).

In the same year, $\mathrm{Xu}$ et al. have patented a synthesis of 5-chloro-1-indanone via the reaction of malonic acid with chlorobenzaldehyde [21]. In the first step, the substrates reacted 
<smiles>[R]c1cc(C(=O)O)c([R])c([R])c1[R]</smiles>

15

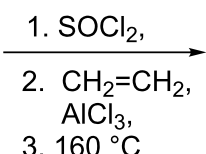

3. $160{ }^{\circ} \mathrm{C}$

Selected examples:<smiles>[R]c1c([R])c([R1])c2c(c1[R])CCC2=O</smiles>

16

16a-d $R^{2}=R^{3}=R^{4}=H ; 16 a R^{1}=F(68 \%) ; 16 b R^{1}=\mathrm{Cl}(73 \%) ; 16 c R^{1}=\operatorname{Br}(59 \%) ; 16 d R^{1}=$ OMe (42\%)

$16 e, f R^{1}=R^{2}=R^{4}=H ; 16 e R^{3}=\mathrm{Cl}(77 \%) ; 16 f R^{3}=$ OMe $(36 \%)$

16g, h R $\mathrm{R}^{1}=\mathrm{R}^{4}=\mathrm{H} ; \mathbf{1 6 g} \mathrm{R}^{2}=\mathrm{R}^{3}=\mathrm{F}(56 \%) ; 16 \mathrm{~h} \mathrm{R}^{2}=\mathrm{R}^{3}=\mathrm{OMe}(41 \%)$

$16 \mathrm{i}, \mathrm{j} \mathrm{R}^{1}=\mathrm{R}^{3}=\mathrm{H} ; 1 \mathbf{1 6 i} \mathrm{R}^{2}=\mathrm{OMe}, \mathrm{R}^{4}=\mathrm{Me}(28 \%) ; 16 \mathrm{j} \mathrm{R}^{2}=\mathrm{Cl}, \mathrm{R}^{4}=\mathrm{Me}(27 \%)$

16k-p R $R^{2} \mathrm{R}^{3}=\mathrm{H} ; \mathbf{1 6 k} \mathrm{R}^{4}=\mathrm{OMe}, \mathrm{R}^{1}=\mathrm{Me}(55 \%) ; 16 \mathrm{I} \mathrm{R}^{1}=\mathrm{F}, \mathrm{R}^{4}=\mathrm{OMe}(47 \%) ; 16 \mathrm{~m} \mathrm{R}^{1}=\mathrm{OH}, \mathrm{R}^{4}=\mathrm{Me}(48 \%)$;

16n $\mathrm{R}^{4}=\mathrm{Cl}, \mathrm{R}^{1}=\mathrm{OH}(57 \%) ; 160 \mathrm{R}^{4}=\mathrm{Me}, \mathrm{R}^{1}=\mathrm{Me}(79 \%) ; 16 \mathrm{p} \mathrm{R} \mathrm{R}^{4}=\mathrm{OMe}, \mathrm{R}^{1}=\mathrm{OH}(39 \%)$

Scheme 6: Synthesis of 1 -indanones 16 from benzoic acids 15.

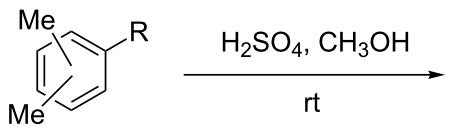

17

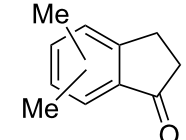

18
$\mathrm{R}=\mathrm{CH}=\mathrm{CHCO}_{2} \mathrm{H}$ or Me positions:

$\mathrm{CH}_{2} \mathrm{CH}_{2} \mathrm{CO}_{2} \mathrm{H} \quad 2 / 3,2 / 4,2 / 5,2 / 6,3 / 4,3 / 5$

Scheme 7: Synthesis of 1-indanones 18 from arylpropionic and 3-arylacrylic acids 17. in the presence of formic acid and diethylamine to form 3-chlorophenylpropionic acid followed by a intramolecular Friedel-Crafts acylation with malonyl chloride in the presence of zinc chloride to give 5-chloro-1-indanone.

New 1-indanone derivatives that may be used as multi-functional drugs for the treatment of Alzheimer's disease have been synthesized by Li et al. [8]. In this synthesis, ferulic acid (23) was hydrogenated in the presence of $\mathrm{Pd} / \mathrm{C}$ catalyst to give the

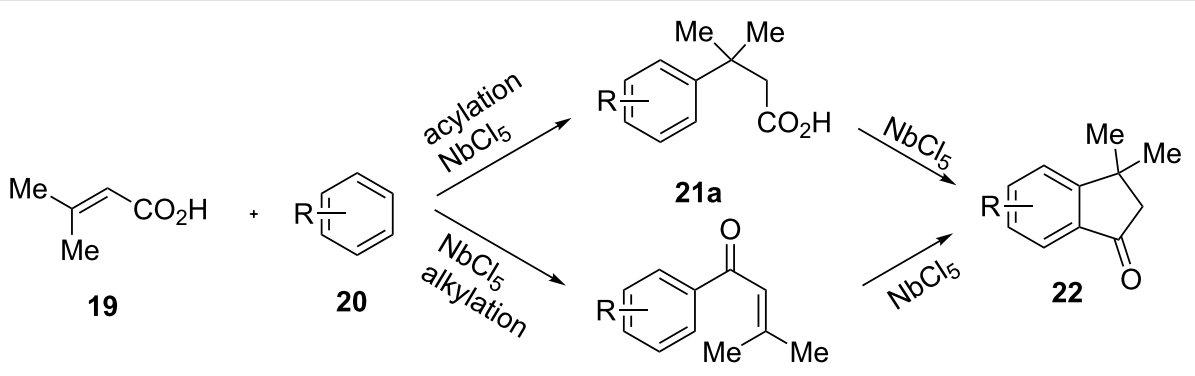

21b

Selected examples<smiles>CC(C)=CC(=O)O</smiles>

19

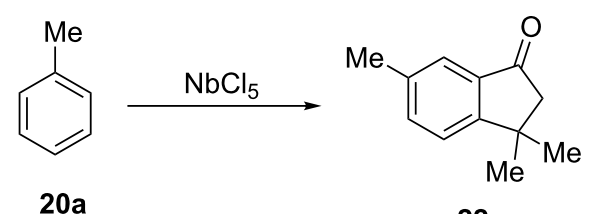

$22 \mathbf{a}$

A. $70 \%$, B. $78 \%$, C. $25 \%$<smiles>CC(C)=CC(=O)O</smiles>

Reaction conditions:

A reflux, $50 \mathrm{~min}$

B $25^{\circ} \mathrm{C}, 80 \mathrm{~min}$

$\mathrm{C} 0^{\circ} \mathrm{C}, 120 \mathrm{~min}$

Scheme 8: The $\mathrm{NbCl}_{5}$-induced one-step synthesis of 1-indanones 22. 
saturated derivative $\mathbf{2 4}$ and then cyclized to the 1-indanone $\mathbf{2 5}$. The latter was then converted to biologically active 1-indanone derivatives $\mathbf{2 6}$ in three steps (Scheme 9). The authors tested activities of the synthesized compounds $\mathbf{2 6}$ for inhibition of cholinesterases (AChE and $\mathrm{BuChE}$ ) and inhibition of amyloid beta $(A \beta)$ self-assembly. The studies have shown that most of the compounds $\mathbf{2 6}$ exhibited a good inhibitory activity against AChE. For instance, compounds $\mathbf{2 6 d}$ and $\mathbf{2 6} \mathbf{i}$ demonstrated $\mathrm{IC}_{50}$ values of 14.8 and $18.6 \mathrm{nM}$, respectively and a remarkably inhibition of $\mathrm{A} \beta$ aggregation.

The environmentally benign synthesis of 1-indanones from 3 -arylpropanoic and 4-arylbutanoic acids has been reported in 2015 by Le et al. [22]. The authors applied a microwaveassisted intramolecular Friedel-Crafts acylation catalyzed by metal triflate in triflate-anion containing ionic liquids. This synthesis proceeded with the goals of green chemistry and allowed to obtain 1-indanones in good yields. Moreover, the metal triflate could be recovered and reused without loss of catalytic activity.
Indatraline that blocks the action of cocaine contains moieties having antidepressant, antihistamine and blood pressurelowering properties. Yun et al. [23] have developed a new method for the synthesis of pure indatraline ((-)-29) in a sequence of reactions starting from carboxylic acid $\mathbf{2 7}$ (Scheme 10).

1.1.2 From acid chlorides: The first synthesis of unsubstituted 1-indanone (2), obtained from the reaction of phenylpropionic acid chloride with aluminum chloride in benzene (90\% yield), has been published in 1927 [24]. In the same year, Mayer and Müller have described a cyclization of unsaturated ketones with acid chlorides leading to the formation of 1-indanones [25].

The use of other acidic catalysts, like $\mathrm{ZnBr}_{2}$ or Nafion ${ }^{\circledR}-\mathrm{H}$ also led to the formation of 1 -indanones [26,27]. Thus, treatment of the acid chloride 30 with Nafion ${ }^{\circledR}-\mathrm{H}$ in refluxing benzene gave unsubstituted 1-indanone (2) in 90\% yield (Scheme 11). In the reaction described above, acid chloride groups reacted with free sulfonic acid groups of Nafion ${ }^{\circledR}-\mathrm{H}$ to generate in situ highly<smiles>COc1cc(/C=C/C(=O)O)ccc1O</smiles>

23<smiles>COc1cc2c(cc1O)C(=O)CC2</smiles>

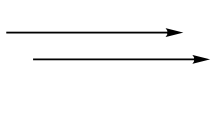<smiles>COc1cc(CCC(=O)O)ccc1O</smiles>

24<smiles>[R]N([R])c1ccc(/C=C2\Cc3cc(OC)c(OC)cc3C2=O)cc1</smiles>

26

26a $n=2, \mathrm{R}^{1}=\mathrm{R}^{2}=\mathrm{Me}(87 \%) ; \mathbf{2 6 b} n=3, \mathrm{R}^{1}=\mathrm{R}^{2}=\mathrm{Me}(79 \%) ; \mathbf{2 6 c} n=5, \mathrm{R}^{1}=\mathrm{R}^{2}=\mathrm{Me}(90 \%)$

26d $n=2, \mathrm{R}^{1}=\mathrm{R}^{2}=\mathrm{Et}(88 \%) ; \mathbf{2 6 e} n=3, \mathrm{R}^{1}=\mathrm{R}^{2}=\mathrm{Et}(74 \%) ; \mathbf{2 6 f} n=5, \mathrm{R}^{1}=\mathrm{R}^{2}=\mathrm{Et}(81 \%) ;$

26g $n=2, \mathrm{R}^{1}=\mathrm{Me}, \mathrm{R}^{2}=\mathrm{H}(85 \%) ; \mathbf{2 6 h} n=2, \mathrm{R}^{1}=\mathrm{Me}, \mathrm{R}^{2}=\mathrm{Et}(66 \%) ;$

26i $n=2, \mathrm{R}^{1}=\mathrm{Me}, \mathrm{R}^{2}=n-\operatorname{Pr}(70 \%) ; \mathbf{2 6 j} n=2, \mathrm{R}^{1}=\mathrm{R}^{2}=n-\operatorname{Pr}(76 \%)$

Scheme 9: Synthesis of biologically active 1-indanone derivatives 26.<smiles>CN[C@H]1C[C@H](c2ccc(Cl)c(Cl)c2)c2ccccc21</smiles>

Scheme 10: Synthesis of enantiomerically pure indatraline ((-)-29). 
reactive mixed anhydrides which cyclized to produce cyclic ketones. In order to complete the catalytic cycle, Nafion ${ }^{\circledR}$-H was regenerated in the acylation step.

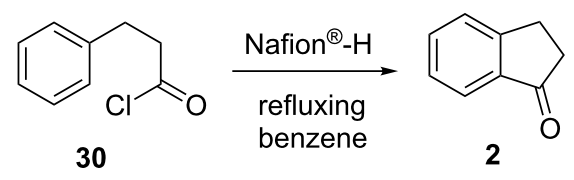

Scheme 11: Synthesis of 1-indanone (2) from the acyl chloride 30.

Investigation of luminescence is a source of valuable information in modern molecular biology, immunology and embryology. An example of a bioluminescent molecule is coelenterazine (luciferin) of which three inhibitors containing an 1-indanone core $\mathbf{3 3}$ have been synthesized to follow the bioluminescence reaction mechanism [28]. The intramolecular Friedel-Crafts acylation of 3-arylpropionic acid derivative $\mathbf{3 1}$ followed by conversion of the acid to the corresponding acyl chloride with thionyl chloride, led to the formation of 1-indanone 32 which was further transformed into the desired inhibitors 33 (Scheme 12).

In the synthesis of 5-hydroxy-1-indanone, Chen and Li reacted 3-chloropropionyl chloride with 2,6-dibromophenol to give 2,6dibromophenyl 3-chloropropionate [29]. Next, the latter was converted to 4,6-dibromo-5-hydroxy-1-indanone in the presence of a Lewis acid and then transformed to 5-hydroxy-1indanone as a result of debromination.

Adrenergic receptors are metabotropic receptors located on cell membranes and stimulated by catecholamines, especially adrenaline and noradrenaline. A new method for the synthesis of the indane 2-imidazole derivative $\mathbf{3 7}$ acting as a strong adrenergic receptor agonist has been proposed by Roberts et al. [30]. In this synthesis, the diacid $\mathbf{3 4}$ was converted to 1-indanone $\mathbf{3 6}$ via the $\mathrm{AlCl}_{3}$ promoted Friedel-Crafts acylation of the acid dichloride $\mathbf{3 5}$. Then, in a sequence of reactions, the 1-indanone $\mathbf{3 6}$ was transformed to $\mathbf{3 7}$ (Scheme 13).

Kabdulov, Amsharov and Jansen have proposed a methodology for the synthesis of fluorinated polyaromatic hydrocarbons<smiles>COc1ccc(-c2ccc(CCC(=O)O)c(Cc3ccccc3)c2)cc1</smiles>

31<smiles>COc1ccc(-c2cc(Cc3ccccc3)c3c(c2)C(=O)CC3)cc1</smiles>

32

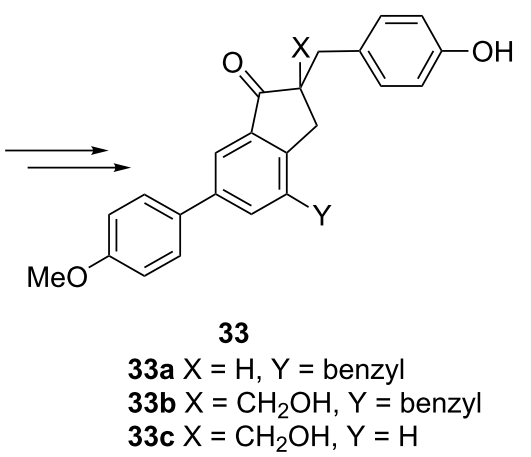

Scheme 12: Synthesis of the mechanism-based inhibitors 33 of coelenterazine.

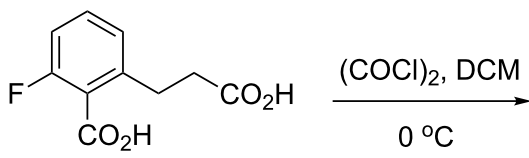

34
1. $\mathrm{AlCl}_{3}, \mathrm{DCM}, 40^{\circ} \mathrm{C}$

2. $\mathrm{MeOH}$<smiles>CC(=O)c1c(F)ccc2c1CCC2=O</smiles>

36<smiles>O=C(Cl)CCc1cccc(F)c1C(=O)Cl</smiles>

35<smiles>Fc1cnn(Cc2c(F)ccc3c2CCC3c2ncc[nH]2)c1</smiles>

37 
(PAH's) via the 1-indanone intermediates 40 [31]. In this synthesis, acids $\mathbf{3 8}$ have been transformed to the corresponding acid chlorides 39, followed by an intramolecular Friedel-Crafts acylation in the presence of $\mathrm{AlCl}_{3}$ in dichloromethane to give the corresponding 1-indanones $\mathbf{4 0}$. The latter were cyclized using $\mathrm{TiCl}_{4}$ in $o$-dichlorobenzene to fluorinated PAHs 41 (Scheme 14).

1.1.3 From esters and diesters: In 1951, Gilmore has demonstrated that use of esters, rather than free arylpropionic acids in phosphoric acid, in the presence of phosphorus pentoxide also led to 1-indanones in equally good or even better yields [32].

Transition metal complexes have been used by Negishi et al. as catalysts in the carbonylative cyclization reaction of carboxylic acid methyl and ethyl esters $\mathbf{4 2}$ which led to the formation of 1 -indanones 43 [33]. This reaction was carried out in acetonitrile, in the presence of triethylamine, under carbon monoxide atmosphere, achieving efficiency in the range of $88-92 \%$, when using lithium, nickel and palladium catalysts (Scheme 15). A general mechanism illustrating the role of transition metal complexes and $\mathrm{CO}$ in this reaction is shown in Scheme 15.

Cyclic esters were also used in the syntheses of 1-indanones. Thus, by adding $\beta$-propiolactone to aluminum chloride in benzene, 1 -indanone has been obtained in $80 \%$ yield. Interestingly, when aluminum chloride was added to the lactone in benzene, the yield of this reaction decreased (30\%) [34].

Irradiation of esters 44 possessing the photoremovable 2,5dimethylphenacyl group in benzene or cyclohexane solutions led to free carboxylic acids 45 (85-95\%) accompanied by 6-methyl-1-indanone (46) which was formed as a byproduct in $5-15 \%$ yields [35]. Irradiation of esters 44 in methanol gave 6-methyl-1-indanone (46) along with 2-(methoxymethyl)-5methylacetophenone (47) and the corresponding free carboxylic acid 45 (Scheme 16).<smiles>[R]c1c(F)cc2c(CC(=O)O)cc3ccc4ccccc4c3c2c1F</smiles>

38a $(90 \%) ; \mathbf{3 8 b}(84 \%)$

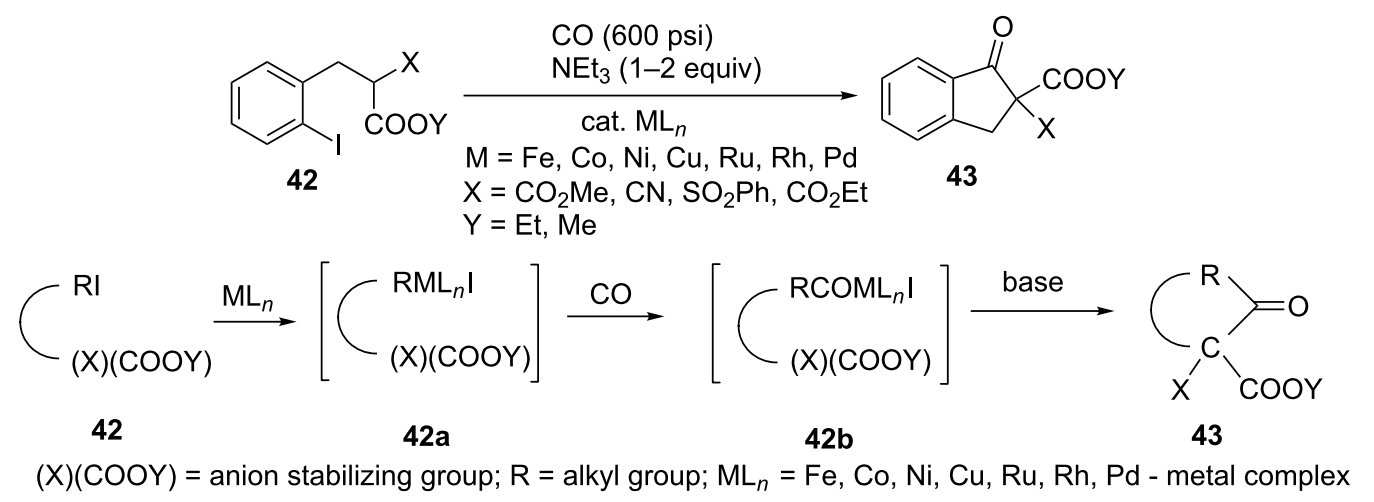




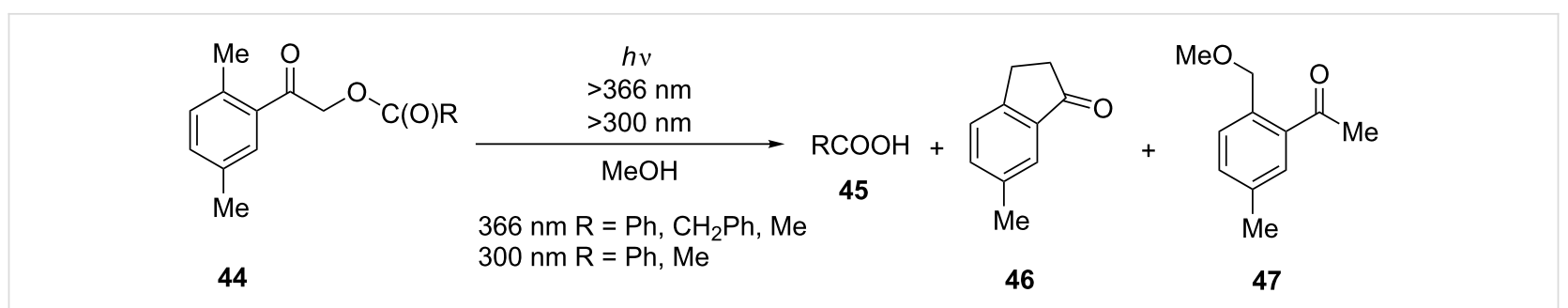

Scheme 16: Synthesis of 6-methyl-1-indanone (46)

The unsubstituted 1-indanone (2) has been synthesized quantitatively by Nakamura, Sugimoto and Ohwada via trifluoromethanesulfonic acid (TFSA)-catalyzed intramolecular cyclization of ester 48 (Scheme 17) [36].<smiles>COC(=O)CCc1ccccc1</smiles>

48

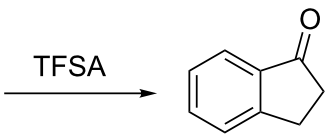

2
Scheme 17: Synthesis of 1-indanone (2) from ester 48.

In 2013, Zhou and Matsuya have proposed an effective method for the preparation of 5,7-dimethoxy-1-indanone [37]. In this synthesis, a mixture of diethyl 2-(3,5-dimethoxybenzyl)malonate and methanesulfonic acid was stirred at $100{ }^{\circ} \mathrm{C}$ for $2 \mathrm{~h}$ and 5,7-dimethoxy-1-indanone was obtained in excellent yield $(95 \%)$.

A new route for the synthesis of an anticancer agent, benzopyronaphthoquinone $\mathbf{5 1}$ from the spiroindanone $\mathbf{5 0}$ has been proposed by Estévez et al. [38]. Thus, starting from 2,2-disubstituted-1-indanone 49, the spiro-1-indanone $\mathbf{5 0}$ was formed via cyclization using $\mathrm{HBr} / \mathrm{AcOH}$ and next converted in a sequence of reactions to the biologically active benzopyronaphthoquinone 51 (Scheme 18).

Endothelins are 21-amino acid peptides with vasoconstrictor properties, produced primarily in the endothelium. They play a key role in vascular homeostasis and are responsible for proper vascular tone and vascular perfusion maintaining. In 2006, Miyaura et al. have synthesized selective endothelin A receptor antagonists $\mathbf{5 5}$ via a formal 1,4-addition of arylboronic acids to $\beta$-aryl- $\alpha, \beta$-unsaturated ketones and esters [39]. Thus, the $\alpha, \beta$ unsaturated diester 52 was coupled with arylboronic acid in the presence of rhodium(I)/Chiraphos ${ }^{\circledR}$ complex as a catalyst to obtain derivative $\mathbf{5 3}$, which next underwent a Claisen condensation to form 1-indanone 54. The latter was further used as a substrate for the synthesis of selective endothelin A receptor antagonist 55 (Scheme 19).

A simple and efficient synthesis of 1-indanones $\mathbf{6 0}$ from methyl vinyl ketone (57) has been proposed by Felpin et al. [40]. In this synthesis, the authors have applied a Heck-reduction-cyclization-alkylation (HRCA) methodology under mild and simple reaction conditions. First, diazonium salts $\mathbf{5 6}$ underwent the Heck reaction with methyl vinyl ketone (57) to give the crosscoupling products $\mathbf{5 8}$ followed by hydrogenation of the latter to give aromatic ketoesters 59. The base-mediated cyclization of the latter in the presence of sodium ethoxide led to the formation of the corresponding 1-indanone anions $\alpha$ to carbonyl, which next were alkylated to give 2-substituted 1-indanones 60 (Scheme 20). This one-pot process utilizing a multi-task palladium catalyst allowed the synthesis of $\mathbf{6 0}$ in yields ranged from 31 to $56 \%$.

1-Indanones exhibit a broad spectrum of biological activity including anti-inflammatory [41], analgesic [42], antimicrobial [43], antiviral [5], anticancer [44] and antimalarial [45] activity. A combination of two or more biologically active moieties may increase or decrease the biological activity. A series of isoxa-<smiles>CCOC(=O)c1ccccc1CC1(C(=O)OCC)Cc2ccccc2C1=O</smiles><smiles>O=C1c2ccccc2CC12Cc1ccccc1C2=O</smiles>

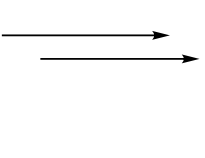

50<smiles>O=C1c2ccccc2C(=O)c2c1oc(=O)c1ccccc21</smiles>

51 


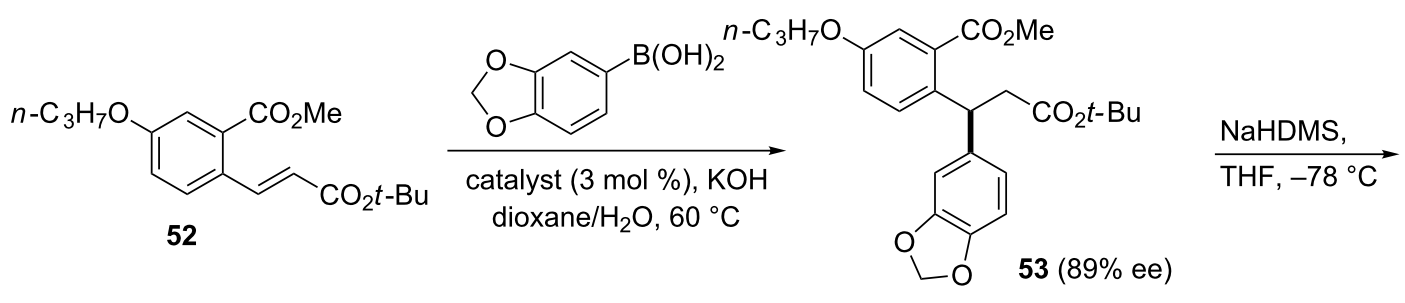

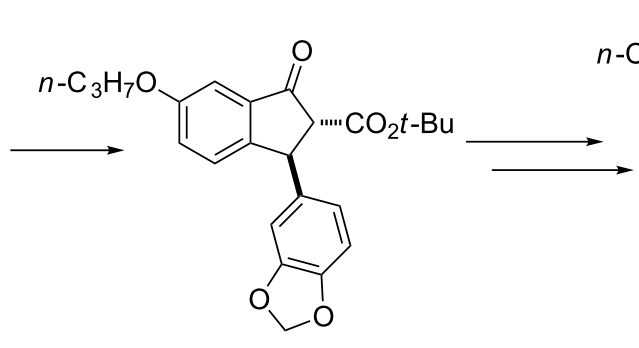

54 (76\%)

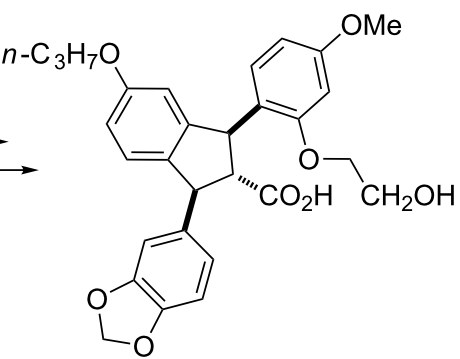

55

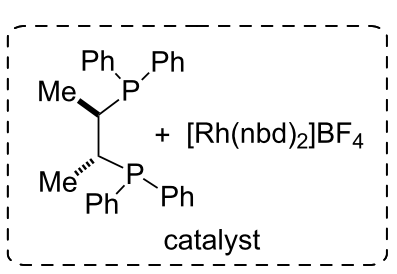

NaHDMS - sodium bis(trimethylsilyl)amide nbd - norbornadiene

Scheme 19: Synthesis of the selective endothelin A receptor antagonist 55.

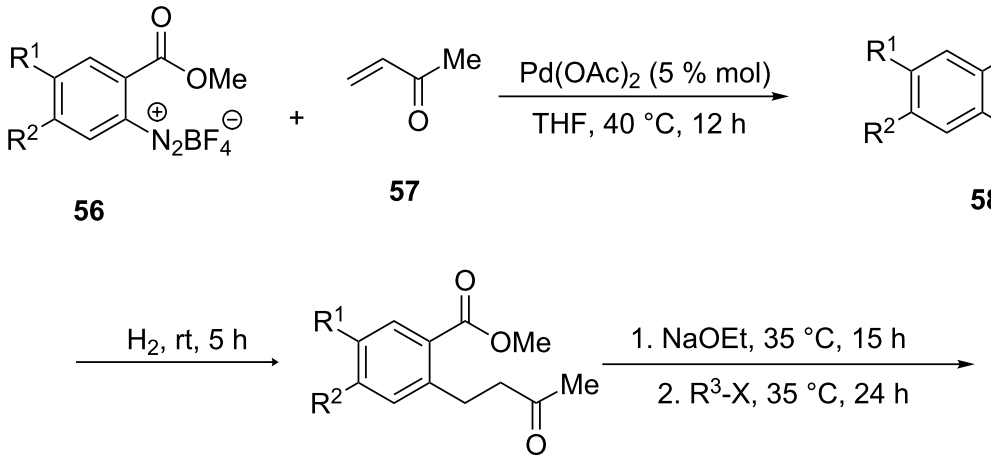

59<smiles>[R]c1cc2c(cc1[R])C(=O)C([R3])(C(C)=O)C2</smiles>

60<smiles>C#CCCCCCc1ccc(I)cc1CCc1ccccc1</smiles>

$60 a-e R^{1}, R^{2}=H \quad 60 a(52 \%), 60 b(43 \%), 60 c(47 \%), \quad 60 d(56 \%), \quad 60$ e $(31 \%)$,

60 'a-d $R^{1}, R^{2}=$ OMe 60'a (40\%), 60'b (33\%), 60'c (31\%), 60'd (47\%)

Scheme 20: Synthesis of 1 -indanones 60 from methyl vinyl ketone (57).

zole fused 1-indanones 64 with increased anti-inflammatory and antimicrobial activity has been synthesized by Giles et al. [46]. In this synthesis, diethyl phthalate $(\mathbf{6 1})$ was reacted with ethyl acetate to obtain indane-1,3-dione (62), followed by a Knoevenagel condensation with a variety aromatic aldehydes to give chalcone derivatives 63 (Scheme 21). The reaction of the latter with hydroxylamine hydrochloride, followed by intramolecular 1,4-addition gave 1-indanone derivatives $\mathbf{6 4 a} \mathbf{a}-\mathbf{l}$ which were further tested for in vitro antibacterial activity against
Escherichia coli and Bacillus subtilis, and antifungal activity against Aspergillus niger and Penicillium notatum. Among the synthesized series of 1-indanone derivatives 64, the highest antibacterial activity was exhibited by derivatives $64 \mathbf{k}$ and $64 \mathbf{l}$, whereas the most potent antifungal activity was revealed for derivatives $\mathbf{6 4 h}$ and $\mathbf{6 4 j}$. The authors have also studied anti-inflammatory properties of these derivatives using the carrageenan induced paw edema method in rats. The anti-inflammatory activity of the synthesized compounds was com- 


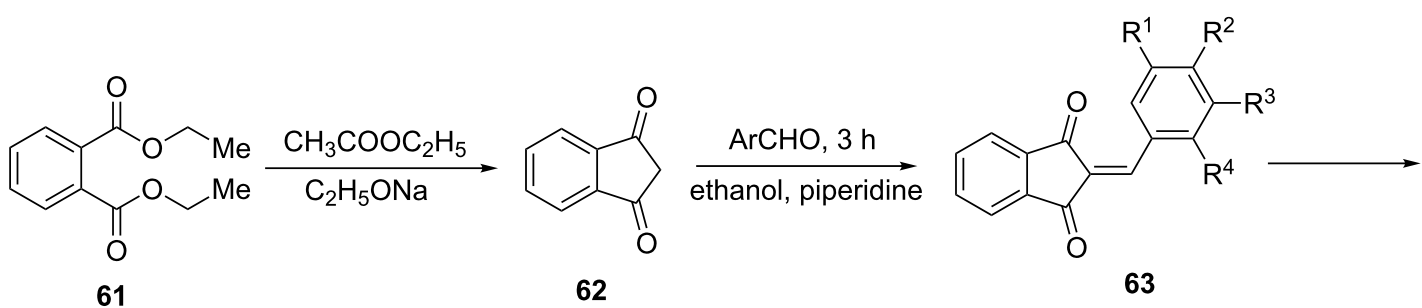<smiles>[R]c1cc(-c2onc3c2C(=O)c2ccccc2-3)c([R])c([R])c1[R]</smiles>

$$
\begin{array}{ll}
\text { 64a } R^{1}=N_{2}, R^{2}=R^{3}=R^{4}=H(72 \%) & \text { 64g } R^{1}=R^{2}=R^{4}=H, R^{3}=O H(73 \%) \\
\text { 64b } R^{1}=R^{3}=R^{4}=H, R^{2}=N O_{2}(71 \%) & \text { 64h } R^{1}=R^{2}=R^{4}=H, R^{3}=O M e(72 \%) \\
\text { 64c } R^{1}=R^{2}=R^{4}=H, R^{3}=N O_{2}(67 \%) & \text { 64i } R^{1}=H, R^{2}=R^{3}=R^{4}=\text { OMe (68\%) } \\
\text { 64d } R^{1}=R^{2}=R^{4}=H, R^{3}=N M e_{2}(72 \%) & \text { 64j } R^{1}=O H, R^{2}=R^{4}=H ; R^{3}=\text { OMe (79\%) } \\
\text { 64e } R^{1}=R^{2}=R^{4}=H, R^{3}=C l(70 \%) & \text { 64k } R^{1}=R^{2}=R^{3}=R^{4}=H(69 \%) \\
\text { 64f } R^{1}=R^{2}=R^{4}=H, R^{3}=\operatorname{Me}(76 \%) & \text { 64I } R^{1}=R^{4}=H, R^{2}=R^{3}=\text { OMe (71\%) }
\end{array}
$$

Scheme 21: Synthesis of 1-indanones 64 from diethyl phthalate 61 .

pared with standard indomethacin (a non-steroidal anti-inflammatory drug used in rheumatoid arthritis treatment). 1-Indanone derivatives $64 \mathrm{k}, 64 \mathrm{j}, 64 \mathrm{f}, 64 \mathrm{~g}$ and $64 \mathrm{i}$ exhibited a stronger inhibition of the paw edema than indomethacin.

The use of Meldrum's acids $\mathbf{6 5}$ is an alternative method for the synthesis of 1-indanones via intramolecular Friedel-Crafts reaction. These compounds are stable at room temperature, easily prepared, functionalized, handled and purified. Thus, the intramolecular Friedel-Crafts acylation of aromatics with Meldrum's acid derivatives $\mathbf{6 5}$, catalyzed by metal trifluoromethanesulfonates such as $\mathrm{Sc}(\mathrm{OTf})_{3}, \mathrm{Dy}(\mathrm{OTf})_{3}, \mathrm{Yb}(\mathrm{OTf})_{3}$, has been reported in 2005 [6]. The studied Meldrum's acid derivatives 65 were functionalized at $\alpha$ - and/or $\beta$-positions by alkyl, haloalkyl, alkenyl, alkynyl, nitrile, ether, thioether, triisopropylsilyl (TIPS) or tert-butyldiphenylsilyl (TBDPS) groups. Depending on the type of catalyst and substrate, 1-indanone derivatives $\mathbf{6 6}$ were obtained in $13-86 \%$ yields (Scheme 22 ). This method was further applied to the synthesis of biologically active compounds, such as 1-tetralones, 1-benzosuberones and donepezil (a potent acetylcholinesterase inhibitor used in the treatment of Alzheimer's disease)

Halo-1-indanones 69 were synthesized from benzyl Meldrum's acids derivatives 67 in two steps [47]. In this synthesis, they underwent microwave-assisted hydrolysis to carboxylic acids 68, followed by chlorosulfonic acid-mediated Friedel-Crafts cyclization to give halo-1-indanones 69 (Scheme 23).
Quaternized Meldrum's acids 70 have been used for the synthesis of 1 -indanones 71 [48]. The reaction was catalyzed by $\mathrm{Sc}(\mathrm{OTf})_{3}$ and proceeded in very good yields (up to $94 \%$ ) (Scheme 24).

1.1.4 From aldehydes and dialdehydes: The stereoselective dimerization reaction of phthalaldehydes $\mathbf{7 2}$, catalyzed by a $N$-heterocyclic carbene is an outstanding protocol for the synthesis of polyhydroxylated spiro- or fused 1-indanones [49]. Thus, the imidazole-based carbene catalyzed the conversion of phthalaldehydes $\mathbf{7 2}$ to dihydroxyspiro[indane-2,1'-isobenzofuran]-3-ones 73, whereas triazole-based carbene catalyzed the conversion of $\mathbf{7 2}$ to cis-trihydroxyindano[2,1-a] indan-5-ones $\mathbf{7 4}$ (Scheme 25).

Another example of the 1-indanone synthesis using $N$-heterocyclic carbenes (NHC) has been described by Gravel et al. $[50,51]$. The benefit of the described reaction was a rapid construction of three new carbon-carbon bonds and a carbon quaternary center with high diastereoselectivity as a consequence of the Stetter-Aldol-Aldol (SAA) reaction sequence. The Stetter-Aldol-Aldol conversion of the phthaldialdehyde derivatives $\mathbf{7 5}$ and $o$-formyl substituted chalcones $\mathbf{7 6}$ using the thiazole based carbene $\mathbf{7 8}$ as a precatalyst allowed to obtain spiro-1,3-indanodiones 77 (Scheme 26). It has been found that the intermediate $\mathbf{7 9}$ underwent the Stetter reaction to form the enolate intermediate 80, which next was transformed to the intermediate $\mathbf{8 1}$ via aldol condensation. The release of NHC 
<smiles>[R]c1cc([R3])cc(C([R])([R])C2C(=O)OC(C)(C)OC2=O)c1</smiles>

65a-k<smiles>[R]c1cc([R])c2c(c1)C([R])([R])CC2=O</smiles>

66a-k

66a $R^{1}=R^{2}=R^{3}=H(13 \%) ; 66 b R^{1}=R^{2}=-\left(C_{2}\right)_{5}, R^{3}=H(56 \%) ; 66 c R^{1}=M e, R^{2}=-\left(C_{2}\right)_{5} C l, R^{3}=H(52 \%)$,

66d $R^{1}=R^{2}=H, R^{3}=$ OMe $(73 \%) ; 66 e R^{1}=H, R^{2}=M e, R^{3}=O M e(77 \%) ; 66 f R^{1}=R^{2}=-\left(C_{2}\right)_{5^{-}}, R^{3}=$ OMe (83\%),

$66 \mathrm{R} \mathrm{R}^{1}=\mathrm{R}^{2}=-\mathrm{CH}_{2} \mathrm{CH}_{2} \mathrm{O}-, \mathrm{R}^{3}=\mathrm{OMe}(79 \%) ; 66 \mathrm{~h} \mathrm{R} \mathrm{R}^{1}=\mathrm{R}^{2}=-\mathrm{CH}_{2} \mathrm{CH}_{2} \mathrm{~S}-, \mathrm{R}^{3}=\mathrm{OMe}(86 \%)$;

66i $R^{1}=R^{2}=\left(\mathrm{CH}_{2}\right)_{2} \mathrm{C}\left(\mathrm{OCH}_{2}\right)_{2}, \mathrm{R}^{3}=\mathrm{OMe}(78 \%), 66 j \mathrm{R}^{1}=\mathrm{R}^{2}=\mathrm{H}, \mathrm{R}^{3}=\mathrm{Me}(52 \%) ; 66 \mathbf{k} \mathrm{R}^{1}=\mathrm{R}^{2}=-\left(\mathrm{CH}_{2}\right)_{5^{-}}, \mathrm{R}^{3}=\mathrm{Me}(75 \%)$<smiles>[R]C([R])(c1ccc(OC)c(OC)c1)C1C(=O)OC(C)(C)OC1=O</smiles>

$65 I-n$<smiles>[R]C1([R])CC(=O)c2cc(OC)c(OC)cc21</smiles>

66I-n<smiles>[R]C([R])(c1cc(OC)ccc1OC)C1C(=O)OC(C)(C)OC1=O</smiles>

$650, p$<smiles>[R2]C1([R2])CC(=O)c2c(OC)ccc(OC)c21</smiles>

66o,p

66I $\mathrm{R}^{1}=\mathrm{R}^{2}=\mathrm{H}(59 \%) ; 66 m \mathrm{R}^{1}=\mathrm{H}, \mathrm{R}^{2}=\mathrm{Me}(68 \%) ;$

66o $\mathrm{R}^{1}=\mathrm{R}^{2}=\mathrm{Me}(32 \%) ; 66 \mathrm{p} \mathrm{R}^{1}=\mathrm{R}^{2}=\operatorname{TBDPS}(63 \%)$ 66n $\mathrm{R}^{1}=\mathrm{R}^{2}=\operatorname{Me}(69 \%)$<smiles>[R7]Oc1c(OC)cccc1C([R])([R])C1C(=O)OC(C)(C)OC1=O</smiles>

$65 r-v$<smiles>[R10]c1c(OC)ccc2c1C([R])([R])CC2=O</smiles>

$66 r-v$

66r $\mathrm{R}^{1}=\mathrm{R}^{2}=\mathrm{H}, \mathrm{R}^{3}=\mathrm{Me}(64 \%) ; 66 s \mathrm{R}^{1}=\mathrm{H}, \mathrm{R}^{2}=\mathrm{R}^{3}=\mathrm{Me}(38 \%) ; 66 \mathrm{R}^{1}=\mathrm{R}^{2}=\mathrm{R}^{3}=\mathrm{Me}(0 \%) ;$ 66u $R^{1}=R^{2}=H, R^{3}=\operatorname{TBDPS}(50 \%) ; 66 v R^{1}=R^{2}=H, R^{3}=\operatorname{TIPS}(45 \%)$

Scheme 22: Synthesis of 1-indanone derivatives 66 from various Meldrum's acids 65<smiles>Cc1ccc(CC2C(=O)OC(C)(C)OC2=O)cc1</smiles>

67

\section{$\underset{\substack{\text { microwaves, } 30 \mathrm{~min} \\>98 \%}}{\stackrel{\mathrm{H}_{2} \mathrm{O} / \mathrm{CH}_{3} \mathrm{CN}(1: 100)}{\longrightarrow}}$}<smiles>[X]c1ccc(CCC(=O)O)cc1</smiles>

68

\section{or PPA, $80^{\circ} \mathrm{C}, 12 \mathrm{~h}$}<smiles>[X]c1ccccc1C(=O)CC</smiles>

69

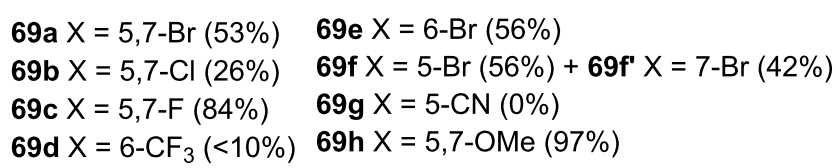

Scheme 23: Synthesis of halo 1-indanones 69.

gave the $\beta$-hydroxy ketone $\mathbf{8 2}$, which was deprotonated to enolate 83. The latter underwent a Michael cyclization reaction to afford 84. Finally, dehydratation of $\mathbf{8 4}$ gave the spiro bisindane product 85 (Scheme 27).

A new method to synthesize 2-benzylidene-1-indanone derivatives 88a-d has been proposed in 2014 by Álvarez-Toledano et al. [52]. These derivatives were obtained from the reaction of $o$-phthalaldehyde (86) with acetophenone 87 (Scheme 28).
Iron(III) complexes of $\mathbf{8 8 a}-\mathbf{d}$ turned out to be promising candidates for potential photovoltaic or luminescence applications.

An intramolecular hydroacylation, catalyzed by nickel( $(0) / N$ heterocyclic carbenes leading to the formation of a variety of 1-indanones and 1-tetralones has been proposed by Ogoshi et al. [53]. Thus, hydroacylation of $o$-allylbenzaldehyde derivatives 89 in the presence of $\left[\mathrm{Ni}(\operatorname{cod})_{2}\right]$ and the $N$-heterocyclic carbene 
<smiles>[R]c1cc(CC2([R])C(=O)OC(C)(C)OC2=O)c([R1])c([R])c1[R]</smiles>

70<smiles>[R]c1c([R])c([R])c2c(c1[R])CC([R8])C2=O</smiles>

71

\section{Selected examples}

71a-g R $\mathrm{R}^{1}=\mathrm{R}^{4}=\mathrm{H}, \mathrm{R}^{2}=\mathrm{R}^{3}=\mathrm{OMe} ; 71 \mathrm{a} \mathrm{R} \mathrm{R}^{5}=\mathrm{Me}(77 \%) ; \mathbf{7 1 b} \mathrm{R}^{5}=\mathrm{Bn}(80 \%) ; \mathbf{7 1 c} \mathrm{R}^{5}=\mathrm{CH}_{2} \mathrm{CH}=\mathrm{CH}_{2}(76 \%) ;$

71d R $\mathrm{R}^{5}=\mathrm{CH}_{2} \mathrm{CCH}(80 \%) ; 71$ e R $\mathrm{R}^{5} \mathrm{CH}_{2} \mathrm{C}_{6} \mathrm{H}_{4}(4-\mathrm{CN})(78 \%) ; 71 \mathrm{R}^{5}=\mathrm{CH}_{2} \mathrm{C}_{6} \mathrm{H}_{4}\left(4-\mathrm{NO}_{2}\right)(81 \%) ; 71 \mathrm{R}^{5}=\mathrm{CH}_{2} \mathrm{C}_{6} \mathrm{H}_{5}(80 \%)$

$71 \mathrm{~h}, \mathbf{i} \mathrm{R}^{2}=\mathrm{R}^{4}=\mathrm{H}, \mathrm{R}^{1}=\mathrm{R}^{3}=\mathrm{OMe} ; 71 \mathrm{~h} \mathrm{R} \mathrm{R}^{5}=\mathrm{Me}(87 \%) ; 71 \mathrm{i} \mathrm{R} \mathrm{R}^{5}=\mathrm{OMe}(80 \%)$

$71 \mathrm{j} \mathrm{R}^{1}=$ OMe, $\mathrm{R}^{2}=\mathrm{R}^{3}=\mathrm{H}, \mathrm{R}^{4}=$ OTBDPS, $\mathrm{R}^{5}=\mathrm{H}(94 \%)$

$71 \mathrm{k}, \mathrm{IR}^{1}=\mathrm{R}^{2}=\mathrm{H} ; \mathbf{7 1 k} \mathrm{R}^{3}=\mathrm{R}^{4}=$ OMe, $\mathrm{R}^{5}=\mathrm{Me}(75 \%) ; 71 \mathrm{I} \mathrm{R}^{3}=$ OMe, $\mathrm{R}^{4}=$ OTBDPS, $\mathrm{R}^{5}=\mathrm{Me}(86 \%)$

TBDPS $=$ tert-butyldiphenylsilyl<smiles>[X]c1cc2c(cc1[X])C(O)C1(OC2=O)OC(O)c2cc([X])c([X])cc21</smiles>

73

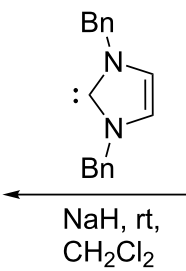

$\mathrm{CH}_{2} \mathrm{Cl}_{2}$

73a $\mathrm{X}=\mathrm{H}(91 \%) \quad 73 \mathbf{c} X=\mathrm{Br}(74 \%)$

$73 b X=M e(63 \%) \quad 73 d X=-C_{2} H_{4}-(58 \%)$<smiles>[X]c1cc(CO)c([X])cc1C=O</smiles>

72

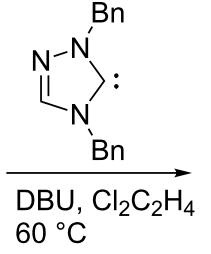

74a X $=\mathrm{H}(85 \%) \quad$ 74d $\mathrm{X}=\mathrm{Br}(92 \%)$

74b $X=M e(71 \%) \quad 74 e X=-C_{2} H_{4}-(72 \%)$ $74 \mathrm{c} X=\mathrm{Cl}(86 \%)$

Scheme 25: Synthesis of spiro- and fused 1-indanones 73 and 74.<smiles>[R7]C=Cc1cccc(C=O)c1</smiles>

75

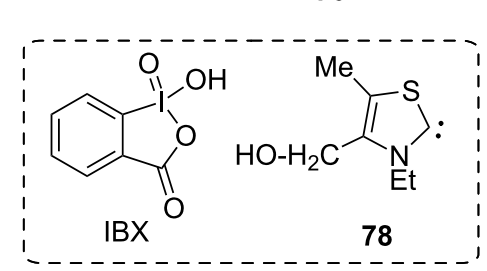

1. $78(30 \mathrm{~mol} \%)$

$\mathrm{DBU}$ (1 equiv)

$\mathrm{CH}_{2} \mathrm{Cl}_{2}(0.5 \mathrm{M})$

$23^{\circ} \mathrm{C}$

2. IBX, $\mathrm{CH}_{3} \mathrm{CN}$

$80^{\circ} \mathrm{C}, 2 \mathrm{~h}$

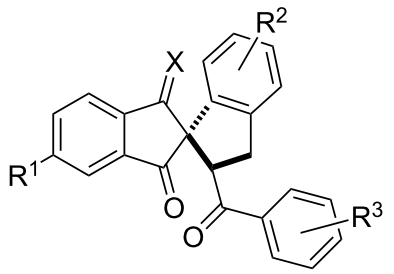

77

77a $\mathrm{R}^{1}=\mathrm{R}^{2}=\mathrm{R}^{3}=\mathrm{H}, \mathrm{X}=\mathrm{H}, \mathrm{OH}(71 \%)$

77b $\mathrm{R}^{1}=\mathrm{R}^{2}=\mathrm{H}, \mathrm{R}^{3}=4-\mathrm{Cl}, \mathrm{X}=\mathrm{O}(58 \%)$

77c $R^{1}=R^{2}=H, R^{3}=4-M e O, X=O(25 \%)$

$77 \mathrm{~d} \mathrm{R}^{1}=\mathrm{MeO}, \mathrm{R}^{2}=\mathrm{R}^{3}=\mathrm{H}, \mathrm{X}=\mathrm{O}(42 \%)$

77 e $R^{1}=R^{3}=H, R^{2}=4-F, X=H, O H(72 \%)$

$77 f R^{1}=F, R^{2}=4-F, R^{3}=4-C l, X=O(50 \%)$

77g R $\mathrm{R}^{1}=\mathrm{R}^{3}=\mathrm{H}, \mathrm{R}^{2}=3-\mathrm{MeO}, X=\mathrm{H}, \mathrm{OH}(36 \%)$

$77 \mathrm{~h} \mathrm{R}^{1}=\mathrm{H}, \mathrm{R}^{2}=4-\mathrm{MeO}, \mathrm{R}^{3}=4-\mathrm{Cl}, \mathrm{X}=\mathrm{O}(75 \%)$ 


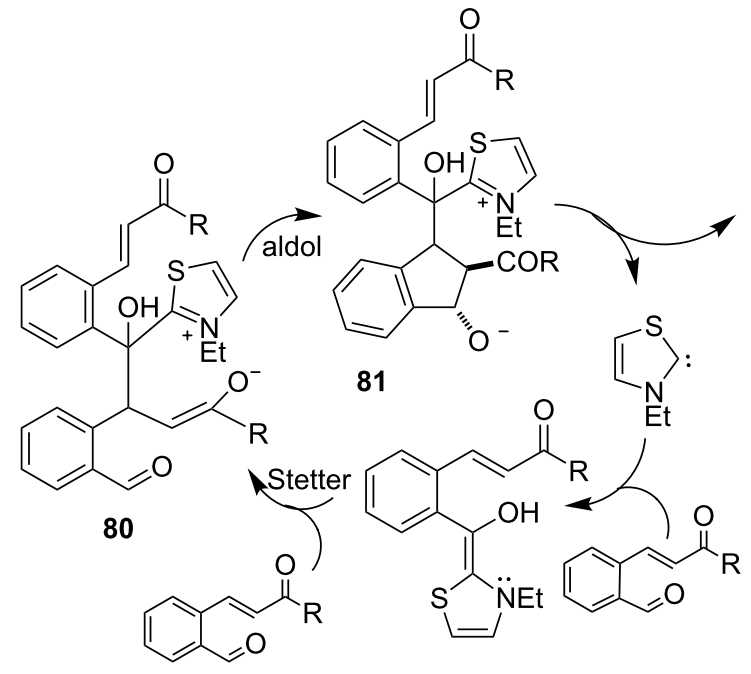

79<smiles>[R]C(=O)/C=C/c1ccccc1C(=O)C1c2ccccc2C(OC)C1C(=O)OCc1ccccc1/C([O-])=C1/c2ccccc2C(O)C1C([R])=O</smiles>

83<smiles>[R5]C(=O)C[C@@H]1c2ccccc2C(=O)[C@@]12C(C([R])=O)=Cc1ccccc12</smiles><smiles>[R]C(=O)C[C@H]1c2ccccc2C(=O)[C@@]12c1ccccc1[C@@H](O)C2C([R])=O</smiles>

84

Scheme 27: Mechanistic pathway for the NHC-catalyzed Stetter-Aldol-Michael reaction.

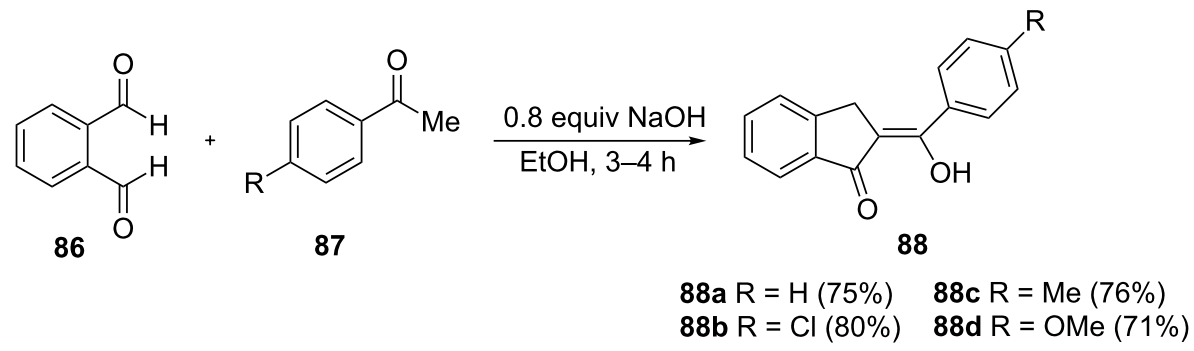

Scheme 28: Synthesis of 2-benzylidene-1-indanone derivatives $\mathbf{8 8 a - d . ~}$

with an $\mathrm{I} t$-Bu substituent gave 1-indanones 90a-i in high yields (Scheme 29). In the case of $\mathbf{9 0}$, it has been proved that this reaction proceeds with participation of Ni-complex 91 isolated in $83 \%$ yield which next was converted to 1 -indanone 90 a via the monomeric complex 92 or its dimer.

$o$-Bromobenzaldehyde $\mathbf{9 3}$, in the presence of a palladium catalyst, underwent intermolecular carbopalladation with alkynes 94, followed by intramolecular nucleophilic vinylpalladation to give indenol derivatives 95 [54]. Further heating of 95 led to isomerization of the double bond to give the corresponding 1-indanones 96 (Scheme 30).

3-Hydroxy-1-indanones 99a-j have been applied in the synthesis of human papilloma virus type 11 (HPV11) inhibitors. These 1-indanones 99 have been synthesized using a $N$-heterocyclic carbene-catalyzed [4+1] annulation utilizing phthalaldehyde (97) and 1,2-diactivated Michael acceptors 98 (Scheme 31) $[55,56]$.

1.1.5 From ketones and 1,2-diketones: Another interesting approach to 1-indanones $\mathbf{1 0 3}$ has been proposed by Wessig et al. [57]. The authors have used a photochemical cyclization of ketones $\mathbf{1 0 0}$ containing good leaving groups $\mathrm{X}$ adjacent to the carbonyl group. As a result of irradiation, 1,4-diradicals 101 have been formed from ketones 100 through $n-\pi^{*}$ excitation followed by 1,5 -hydrogen migration involving the $o$-alkyl substituent. 1,5-Diradicals $\mathbf{1 0 2}$ were formed as a result of elimination of acid HX from 1,4-diradicals 101. Finally, 1,5-diradicals $\mathbf{1 0 2}$ underwent cyclization to give 1-indanones 103 in good yields and 2-alkylidene benzo[c]furan derivatives $\mathbf{1 0 4}$ as byproducts (Scheme 32). 
<smiles>[R2]C=CCc1cccc(C=O)c1C=O</smiles>

$89 a-h R^{2}=H$

89i $R^{2}=P h$<smiles>[R]C1Cc2ccccc2C1=O</smiles>

90a-i

Selected examples

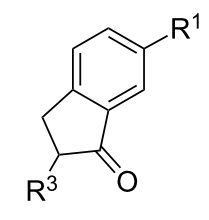

90a-d R $\mathrm{R}^{3}=\mathrm{Me}$

$90 a R^{1}=H(5 h, 98 \%)$

90b R ${ }^{1}=$ OMe (6h, 97\%)

90c R $R^{1}=F(5 h, 75 \%)$

$90 \mathrm{~d} \mathrm{R} \mathrm{R}^{1}=\mathrm{Cl}(6 \mathrm{~h},<1 \%)$<smiles>[R]c1cccc2c1C(=O)C([R])C2</smiles>

90e-g R $\mathrm{R}^{3}=\mathrm{Me}$

90e $R^{1}=\operatorname{Bn}(1 \mathrm{~h}, 99 \%)$

90f $\mathrm{R}^{1}=\mathrm{SiEt}_{3}$ (2 h, 99\%)

$90 \mathrm{~g} \mathrm{R}^{1}=\mathrm{CH}_{2}-\mathrm{CH}=\mathrm{CH}_{2}(5 \mathrm{~h}, 93 \%)$<smiles>CC1Cc2c(ccc3ccccc23)C1=O</smiles>

90h (6 h, 92\%)<smiles>O=C1c2ccccc2CC1Cc1ccccc1</smiles>

$90 \mathrm{i}(24 \mathrm{~h}, 54 \%)$

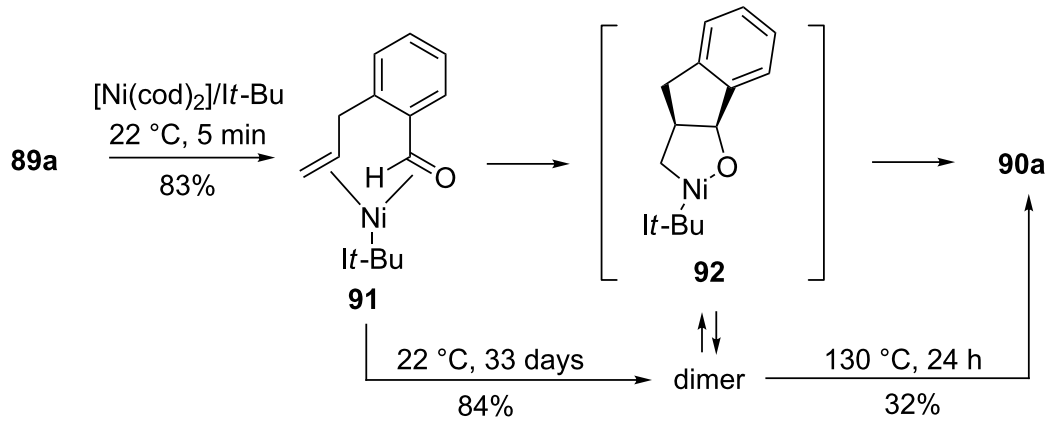

Scheme 29: Synthesis of 1-indanone derivatives $\mathbf{9 0 a - i}$.<smiles>[R]c1ccc(Br)c(C=O)c1</smiles>

93

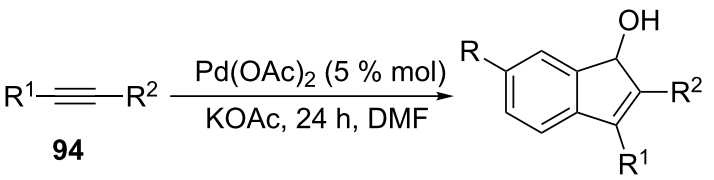

95

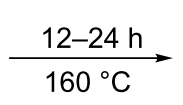<smiles>[R]c1ccc2c(c1)C(=O)C([R])C2[R]</smiles>

96 96a $R=H, R^{1}=R^{2}=n-\operatorname{Pr}(68 \%)$

96b $\mathrm{R}=\mathrm{H}, \mathrm{R}^{1}=-\left(\mathrm{CH}_{2}\right)_{10}(0 \%)$

96c $\mathrm{R}=\mathrm{H}, \mathrm{R}^{1}=\mathrm{Me}, \mathrm{R}^{2}=t-\mathrm{Bu}(69 \%)$

96d $\mathrm{R}=\mathrm{H}, \mathrm{R}^{1}=\mathrm{Ph}, \mathrm{R}^{2}=t-\mathrm{Bu}(73 \%)$

96e $\mathrm{R}=\mathrm{OMe}, \mathrm{R}^{1}=\mathrm{Ph}, \mathrm{R}^{2}=t-\mathrm{Bu}(0 \%)$

Scheme 30: Synthesis of 1-indanones 96 from o-bromobenzaldehydes 93 and alkynes 94.

Chiral 3-aryl-1-indanones $\mathbf{1 0 7}$ have been synthesized via rhodium-catalyzed asymmetric cyclization of pinacolborane chalcone derivatives 105 using $(R)$-MonoPhos ${ }^{\circledR}$ as a chiral ligand [58]. In this reaction, a wide variety of 1-indanones $\mathbf{1 0 7}$ were obtained in high yields and up to $95 \%$ enantiomeric excess (Scheme 33). 


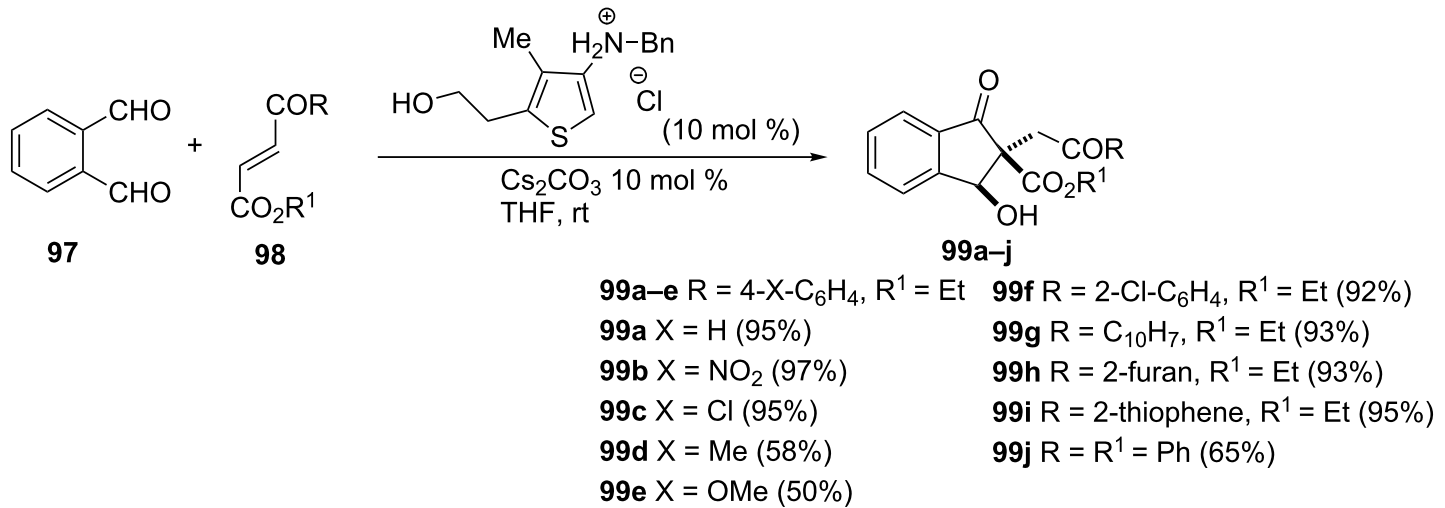

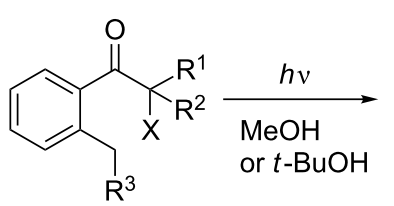

100<smiles>[R][Y]1(CC)c2ccccc2C(O)[Y]1([R])[R]</smiles>

101

$X=\mathrm{OMs}, \mathrm{OTs}, \mathrm{OPO}(\mathrm{OEt})_{2}, \mathrm{OCOO} t$-Bu, OCOOEt, Ac $\mathrm{R}^{2}=\mathrm{H}, \mathrm{Me}$

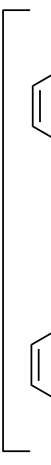

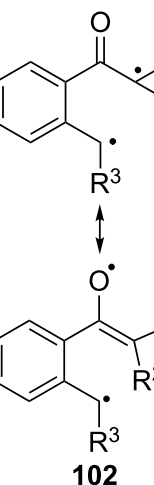<smiles>[R]C1C(=O)c2cc(C)ccc2C1[R]</smiles><smiles>[R]C=C1OCc2ccccc21</smiles>

104 104a $R^{1}=$ COOEt $(67 \%)$; 104b R $\mathrm{R}^{1}=\mathrm{CON}\left(\mathrm{CH}_{2}\right)_{4}$

103a $R^{1}=H, R^{3}=H(42 \%) ; 103 b R^{1}=M e, R^{3}=H(73 \%) ;$ 103c $R^{1}=E t, R^{3}=H(69 \%) ; 103 d R^{1}=P h, R^{3}=H(66 \%) ;$ 103e $R^{1}=$ COOEt, $R^{3}=H(82 \%)$; 103f $\mathrm{R}^{1}=\mathrm{CON}\left(\mathrm{CH}_{2}\right)_{4}, \mathrm{R}^{3}=\mathrm{H}(70 \%)$; 103g R $\mathrm{R}^{1}=\mathrm{iPr}, \mathrm{R}^{3}=\mathrm{H}(46 \%) ; 103 \mathrm{~h} \mathrm{R}^{1}=\mathrm{H}, \mathrm{R}^{3}=\mathrm{Me}(0 \%)$; 103i $R^{1}=H, R^{3}=$ COOMe $(62 \%)$;

103j $R^{1}=H, R^{3}=C N(40 \%)$

Scheme 32: Photochemical preparation of 1-indanones 103 from ketones 100<smiles>[Y]C=CC(=O)c1ccccc1B1OC(C)(C)C(C)(C)O1</smiles>

(E)-105
$\left[\mathrm{Rh}\left(\mathrm{C}_{2} \mathrm{H}_{4}\right)_{2} \mathrm{Cl}\right]_{2}(2.5 \mathrm{~mol} \%)$ $(R)$-MonoPhos ${ }^{\circledR}(10 \mathrm{~mol} \%)$ $\mathrm{K}_{3} \mathrm{PO}_{4}(1.5 \mathrm{M}) /$ toluene, $80{ }^{\circ} \mathrm{C}$

106<smiles>[R]c1ccc2c(c1)C(=O)CC2[Al]</smiles>

107

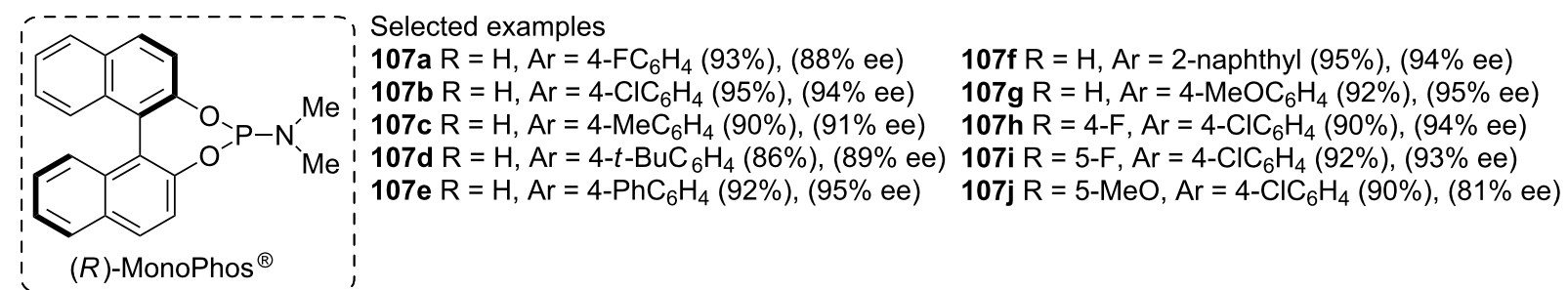

Scheme 33: Synthesis of chiral 3-aryl-1-indanones 107. 
2-Methylbenzil (108) has been converted to 2-hydroxy-2phenylindan-1-one (109) as a result of photochemical isomerization, in $90 \%$ yield (Scheme 34) [59].

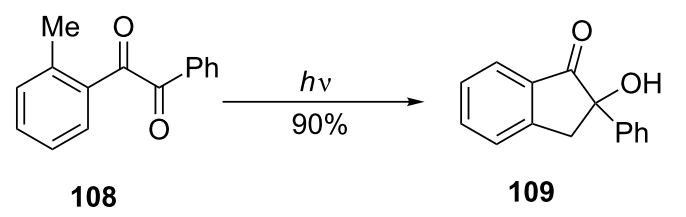

Scheme 34: Photochemical isomerization of 2-methylbenzil 108

Wagner et al. have reported that hexaisopropyl-, hexaethyl- and hexamethylbenzils 110a-c photocyclized to the corresponding 2-hydroxy-1-indanones 111a-c (Scheme 35) [60].

The chromium $\eta^{6}$-1,2-dioxobenzocyclobutene complex 112 could be converted into 1-indanones 113 and $\mathbf{1 1 4}$ by addition of vinyllithium derivatives, followed by a double anionic oxyCope rearrangement under mild conditions (Scheme 36) [61]. The derivative 114 turned out to be particularly interesting because of its application in the synthesis of anticancer compounds [62].

1.1.6 From 1,3-dienones: The major role in the synthesis of 1-indanones plays the Nazarov reaction of 1,3-dienones in which one of two double bonds is derived from the aromatic system. Nakiterpiosin (117) is a marine sponge metabolite which demonstrates a potent cytotoxicity against the P388 leukemic cell line. The photo-variant of the Nazarov cyclization has been applied as one of the steps in the total synthesis of nakiterpiosin (117, Scheme 37) [63]. Starting from substrate 115, 1-indanone 116 was isolated in $60 \%$ yield and further used in the synthesis of the natural product.

Hexamethylenetetramine (HMTA) is a commonly used promoter of aryl alkyl ketones in the Mannich reaction which

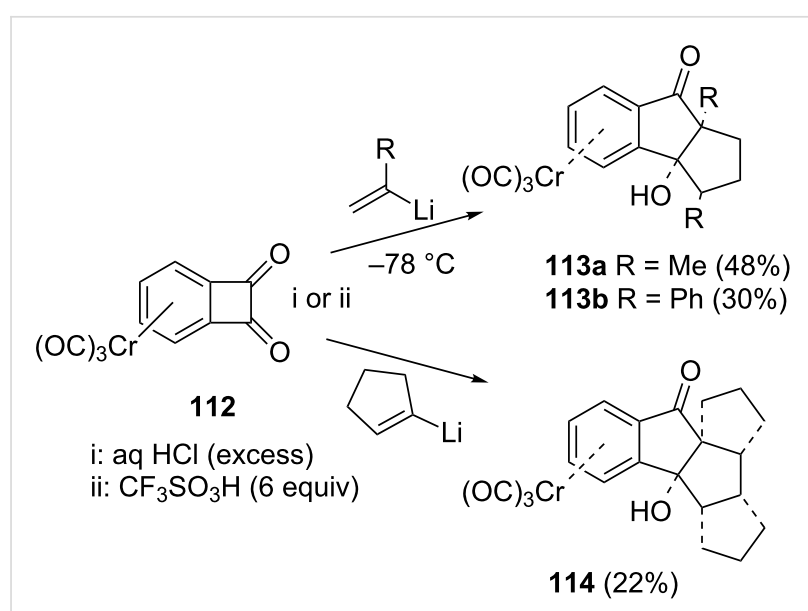

Scheme 36: Synthesis of 1-indanone derivatives 113 and 114 from $\eta^{6}-1,2$-dioxobenzocyclobutene complex 112.

has been applied in the synthesis of $\alpha, \beta$-unsaturated ketones 119 [64]. The HMTA/acetic anhydride-promoted $\alpha$-methylenation of compounds 118 followed by cyclization of the resulting enones 119 allowed to obtain a series of 2-alkyl-1-indanones $\mathbf{1 2 0}$ in very good yields (Scheme 38).

A stereoselective, catalytic, tandem transformation of $\alpha, \beta$-unsaturated arylketones $\mathbf{1 2 1}$ to fluorine-containing 1-indanone derivatives 123 via the Nazarov cyclization followed by electrophilic fluorination, has been described in 2007 by Ma et al. [65]. This reaction was catalyzed by $\mathrm{Cu}(\mathrm{II})$ triflate and proceeded in the presence of $N$-fluorobenzenesulfonimide (NFSI) 122 as a fluorinating reagent (Scheme 39).

Scientists are tirelessly exploring for better anticancer pharmaceuticals. Negi et al. have joined these efforts and proposed a synthesis of 2-benzylidene-1-indanones, which exhibited a strong cytotoxicity against four human cancer cell lines: breast (MCF-7), colon (HCT), leukemia (THP-1) and lung (A549) with $\mathrm{IC}_{50}$ values in the range of $10-880 \mathrm{nM}$ [66]. The synthesized compounds have also shown a strong inhibition of tubulin<smiles>[R]c1cc([R])c(C(=O)C(=O)c2c([R])cc([R])cc2[R])c([R])c1</smiles>

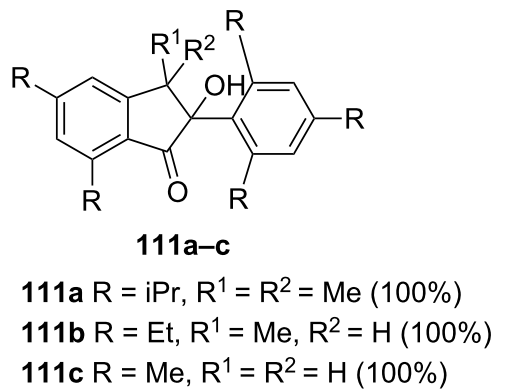




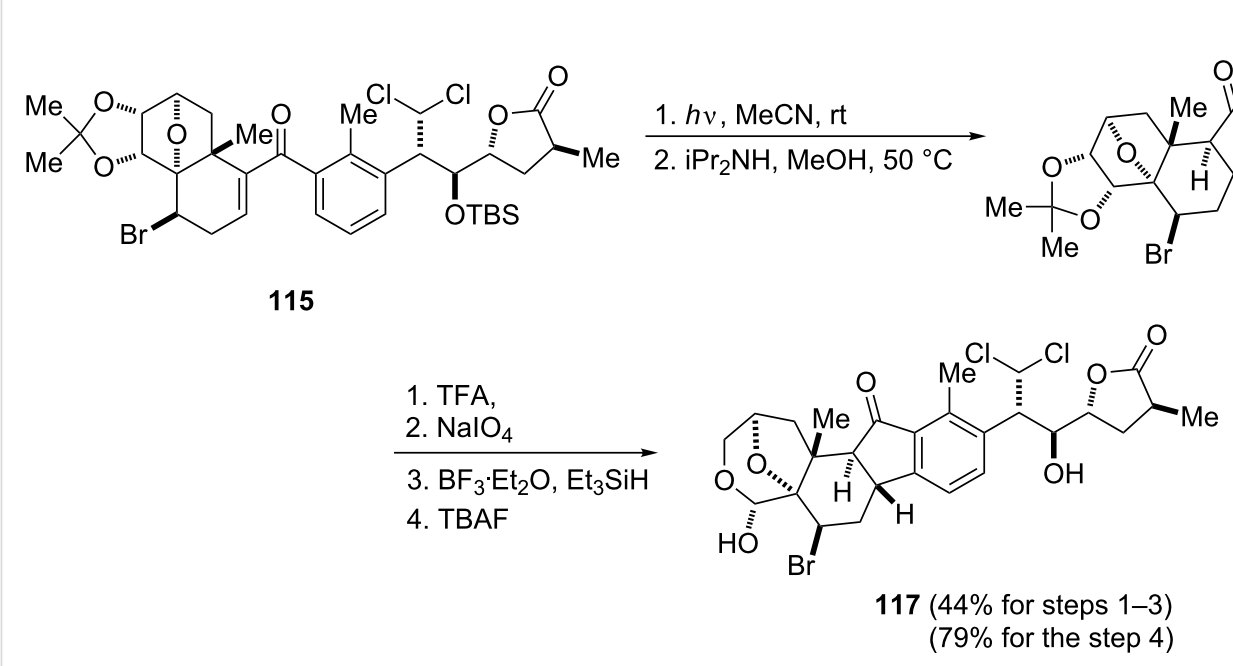

Scheme 37: Synthesis of nakiterpiosin (117).

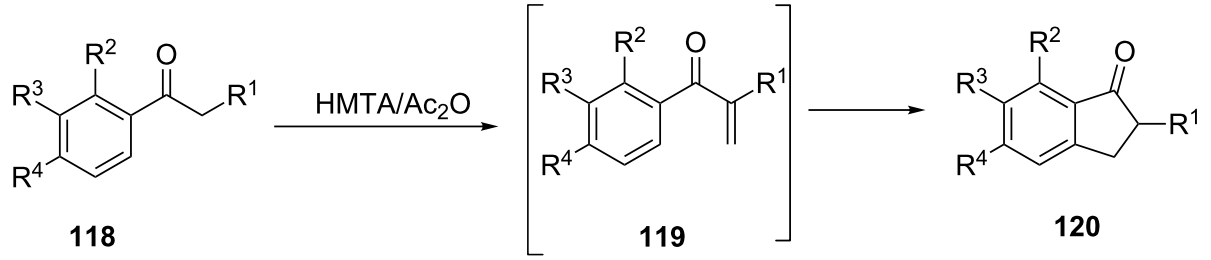

120a $R^{1}=M e, R^{2}=R^{3}=R^{4}=H(82 \%) ; 120 b R^{1}=E t, R^{2}=R^{3}=R^{4}=H(80 \%) ; 120 c R^{1}=n-B u, R^{2}=R^{3}=R^{4}=H(86 \%) ;$

120d $R^{1}=M e, R^{2}=R^{4}=H, R^{3}=B r(82 \%) ; 120 e R^{1}=n-P r, R^{2}=R^{3}=C l, R^{4}=$ OMe (84\%)

Scheme 38: Synthesis of 2-alkyl-1-indanones 120

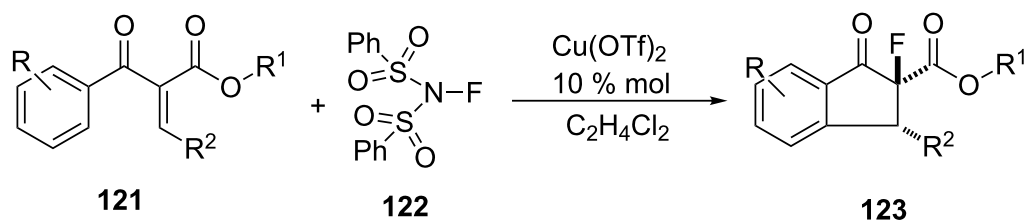

123a-h R $=6,7-\left(\mathrm{OCH}_{2} \mathrm{O}\right), \mathrm{R}^{1}=\mathrm{Me}$

123a $\mathrm{R}^{2}=4-\mathrm{MeO}-\mathrm{C}_{6} \mathrm{H}_{4}(95 \%, t / c=32: 1)$

123i,j $\mathrm{R}=\mathrm{H}, \mathrm{R}^{1}=\mathrm{Me}$

123b $\mathrm{R}^{2}=2-\mathrm{MeO}-\mathrm{C}_{6} \mathrm{H}_{4}(67 \%, t / c=24: 1)$

123i R $=4-\mathrm{Cl}-\mathrm{C}_{6} \mathrm{H}_{4}(82 \%, t / c=24: 1)$

123c $\mathrm{R}^{2}=3-\mathrm{MeO}-\mathrm{C}_{6} \mathrm{H}_{4}(67 \%, t / c=18: 1)$

123j $\mathrm{R}^{2}=4-\mathrm{NO}_{2}-\mathrm{C}_{6} \mathrm{H}_{4}(84 \%, t / c=19: 1)$

123d R $\mathrm{R}^{2}=\mathrm{C}_{6} \mathrm{H}_{5}(35 \%, t / c=19: 1)$

123k $\mathrm{R}=7-\mathrm{MeO}, \mathrm{R}^{1}=\mathrm{Et}, \mathrm{R}^{2}=2,4,6-(\mathrm{MeO})_{3} \mathrm{C}_{6} \mathrm{H}_{2}(85 \%, t / c=19: 1)$

123e $\mathrm{R}^{2}=4-\mathrm{Me}-\mathrm{C}_{6} \mathrm{H}_{4}(45 \%, t / c=15: 1)$

123I $\mathrm{R}=7-\mathrm{Me}, \mathrm{R}^{1}=\mathrm{Et}, \mathrm{R}^{2}=2,4,6-(\mathrm{MeO})_{3} \mathrm{C}_{6} \mathrm{H}_{2}(63 \%, t / c=3: 1)$

123f $\mathrm{R}^{2}=3,4,5-(\mathrm{MeO})_{3}-\mathrm{C}_{6} \mathrm{H}_{2}(82 \%, t / c=24: 1)$

$123 \mathrm{~m} \mathrm{R}=7,8-(\mathrm{MeO})_{2}, \mathrm{R}^{1}=\mathrm{Et}, \mathrm{R}^{2}=2,4,6-(\mathrm{MeO})_{3} \mathrm{C}_{6} \mathrm{H}_{2}(58 \%, t / c=49: 1$

123g R $\mathrm{R}^{2}=2,4,6-(\mathrm{MeO})_{3}-\mathrm{C}_{6} \mathrm{H}_{2}(78 \%, t / c=32: 1)$

$123 \mathrm{n} R=7,8-\mathrm{C}_{6} \mathrm{H}_{4}, \mathrm{R}^{1}=\mathrm{Me}, \mathrm{R}^{2}=2,4,6-(\mathrm{MeO})_{3} \mathrm{C}_{6} \mathrm{H}_{2}(63 \%, t / c=24: 1)$

123h $\mathrm{R}^{2}=\mathrm{iPr}(15 \%, t / c=32: 1)$

t/c: trans/cis

Scheme 39: Synthesis of fluorine-containing 1-indanone derivatives 123.

polymerase with $\mathrm{IC}_{50}$ values in the range of $0.62-2.04 \mu \mathrm{M}$. In this synthesis, the chalcone $\mathbf{1 2 4}$ underwent a Nazarov cyclization in the presence of trifluoroacetic acid to give 1-indanone 125. 2-Benzylidene-1-indanones $\mathbf{1 2 6 a}-\mathbf{0}$ were obtained by a
Knoevenagel condensation of 1-indanone 125 with various aromatic aldehydes. Hydrogenolysis of 2-benzylidene-1-indanone 126b using $\mathrm{Pd} / \mathrm{C}$ allowed to obtain 2-benzyl substituted 1-indanone 127 (Scheme 40). 


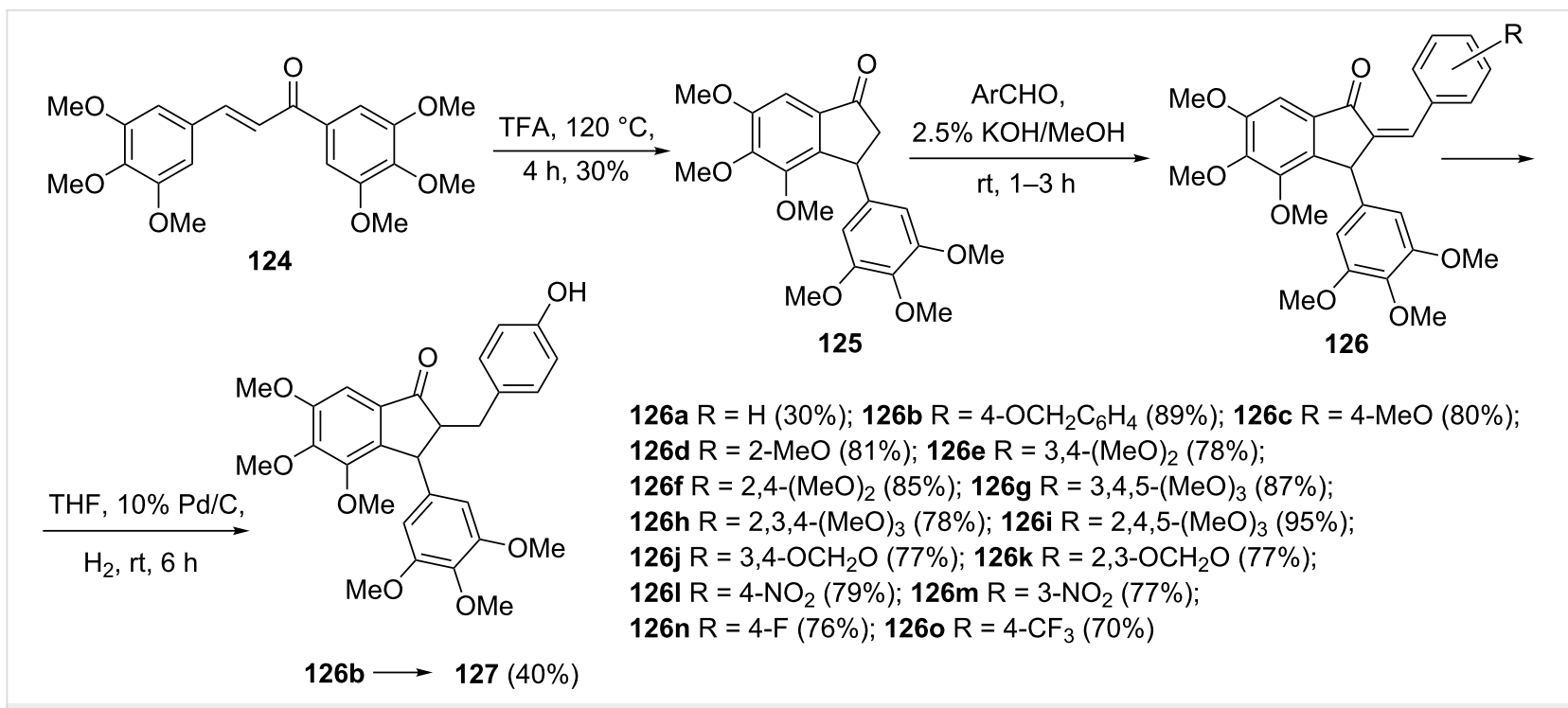

Scheme 40: Synthesis of 2-benzylidene and 2-benzyl-1-indanones 126, 127 from the chalcone 124.

2-Bromo-6-methoxy-3-phenyl-1-indanone 130, as an interesting bromo reagent for further transformations, has been synthesized from chalcone $\mathbf{1 2 8}$ [67]. In this synthesis, a Nazarov reaction of chalcone 128, in the presence of trifluoroacetic acid, gave 6-methoxy-3-phenyl-1-indanone $\mathbf{1 2 9}$ in $88 \%$ yield followed by the reaction with bromine in diethyl ether to give 2-bromo-6-methoxy-3-phenyl-1-indanone (130, Scheme 41).

An efficient microwave-assisted synthesis of 1-indanones 132a-s related to combretastatin A-4 has been proposed by Lawrence et al. [68]. Two of the indanones were obtained via a Nazarov cyclization of chalcones $\mathbf{1 3 1}$ without using microwaves, in the presence of trifluoroacetic acid (TFA) at $120^{\circ} \mathrm{C}$ (4 hours). The authors have proved that the microwave heating would significantly shorten the reaction time up to 20 minutes under the same reaction conditions (TFA, $120^{\circ} \mathrm{C}$, Scheme 42). The cell growth inhibitory properties of the synthesized 1-indanones 132a-s were also investigated on the K562 human chronic myelogenous leukaemia cell line. The strongest cytotoxicity against the K562 cell line showed the following compounds: 132a, 132b, 132d, 132f, 132g, 132i, 132k, 132m, $132 \mathrm{n}, 132 \mathrm{o}$ with $\mathrm{IC}_{50}$ values in the range of $0.08-2.8 \mu \mathrm{M}$. The compound 132b demonstrated the greatest resemblance to combretastatin A-4.

In 2008, Frontier et al. have examinated the impact of the dienone substitution in Nazarov cyclizations [69]. They synthesized a series of the Nazarov substrates 133 with electron-donating substituents at $\mathrm{C}-2$ and electron-withdrawing substituents at $\mathrm{C}-4$. By using catalytic amounts of $\mathrm{Cu}(\mathrm{OTf})_{2}$ or $\mathrm{Cu}\left(\mathrm{ClO}_{4}\right)_{2}$ as Lewis acids, cyclic products $\mathbf{1 3 4}-\mathbf{1 3 7}$ have been obtained as single diastereoisomers in high yields (Figure 3 ). It has been proven that the reactivity and the selectivity of this cyclization can be controlled by positioning of the dienone $\mathbf{1 3 3}$ substituents. In the previous studies of the reductive Nazarov cyclization, similar results were obtained - two $E$ and $Z$ dienone isomers were converted into one diastereoisomeric product $[70,71]$.

A dicationic iridium(III)-catalyzed Nazarov cyclization has been applied for the synthesis of functionalized 1-indanones and their heteroatom analogues 138-142 which may be further converted into biologically active compounds (Figure 4) [72]. Products 138-142 were obtained by electrocyclization of the sub-<smiles>COc1cccc(C(=O)/C=C/c2ccccc2)c1</smiles>

128

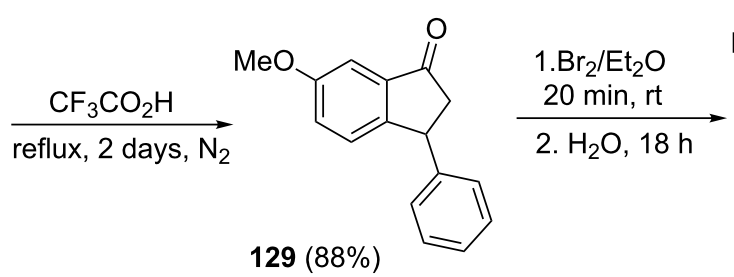

$129(88 \%)$<smiles>COc1ccc2c(c1)C(=O)C(Br)C2c1ccccc1</smiles>

130 
<smiles>[R]C=CC(=O)c1cc([R1])c(OC)c(OC)c1</smiles>

131 $\frac{\text { TFA, microwaves, } 100 \mathrm{~W}}{120^{\circ} \mathrm{C}, 20 \mathrm{~min}}$

132a-n $\mathrm{R}^{1}=$ OMe

132a $\mathrm{R}^{2}=4-\mathrm{MeO}-\mathrm{C}_{6} \mathrm{H}_{4}(60 \%) ; 132 b \mathrm{R}^{2}=3-\mathrm{OH}-4-\mathrm{MeO}-\mathrm{C}_{6} \mathrm{H}_{3}(56 \%) ;$

132c $\mathrm{R}^{2}=4-\mathrm{Cl}-\mathrm{C}_{6} \mathrm{H}_{4}(53 \%) ; 132 \mathrm{~d} \mathrm{R} \mathrm{R}^{2}=3-\mathrm{Br}-4-\mathrm{MeO}-\mathrm{C}_{6} \mathrm{H}_{3}(59 \%)$;

132e $\mathrm{R}^{2}=3,4-(\mathrm{Cl})_{2}-\mathrm{C}_{6} \mathrm{H}_{3}(69 \%) ; 132 f \mathrm{R}^{2}=3,4-\left(\mathrm{OCH}_{2} \mathrm{O}\right)-\mathrm{C}_{6} \mathrm{H}_{3}(54 \%)$;

132g R ${ }^{2}=4-\mathrm{Br}-\mathrm{C}_{6} \mathrm{H}_{4}(33 \%) ; 132 \mathrm{~h} \mathrm{R}^{2}=4-\mathrm{CH}_{2} \mathrm{C}(\mathrm{O}) \mathrm{OH}-\mathrm{C}_{6} \mathrm{H}_{4}(26 \%)$;

132i $\mathrm{R}^{2}=2,3,4-(\mathrm{MeO})_{3}-\mathrm{C}_{6} \mathrm{H}_{2}(25 \%) ; 132 \mathrm{j} \mathrm{R}^{2}=2,6-(\mathrm{Cl})_{2}-\mathrm{C}_{6} \mathrm{H}_{3}(95 \%)$;

132k R $\mathrm{R}^{2}=2,4-(\mathrm{Cl})_{2}-\mathrm{C}_{6} \mathrm{H}_{3}(83 \%) ; 132 \mathrm{I} \mathrm{R}^{2}=\mathrm{C}_{6} \mathrm{H}_{3}(23 \%)$;

$132 m R^{2}=3-\mathrm{NO}_{2}-4-\mathrm{MeO}-\mathrm{C}_{6} \mathrm{H}_{3}(39 \%) ; 132 n \mathrm{R}^{2}=3-\mathrm{F}-4-\mathrm{MeO}-\mathrm{C}_{6} \mathrm{H}_{4}(37 \%)$<smiles>[R]c1c(OC)c(OC)cc2c1C([R])CC2=O</smiles>

$132 \mathrm{a}-\mathrm{s}$

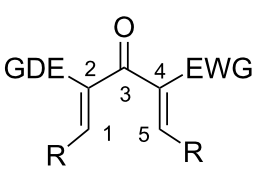

133<smiles>[R]C1C(=O)c2cc3c(cc2C1[Y16])OCO3</smiles>

136<smiles>N#[Y16]C1CC(=O)c2cc3c(cc21)OCO3</smiles>

$134(82 \%)$<smiles>CC(=O)C1C(=O)c2cc3c(cc2[C@H]1[14NH+]=[N-])OCO3</smiles>

$135(90 \%)$ 1320-s $\mathrm{R}^{1}=\mathrm{H}$

$1320 \mathrm{R}^{2}=4-\mathrm{MeO}-\mathrm{C}_{6} \mathrm{H}_{4}(7 \%)$;

132p R $\mathrm{R}^{2}=4-\mathrm{Br}-\mathrm{C}_{6} \mathrm{H}_{4}(52 \%)$;

132q R $\mathrm{R}^{2}=4-\mathrm{Cl}-\mathrm{C}_{6} \mathrm{H}_{4}(34 \%)$;

$132 \mathrm{r} \mathrm{R}^{2}=2,6-(\mathrm{Cl})_{2}-\mathrm{C}_{6} \mathrm{H}_{3}(71 \%)$;

132s $\mathrm{R}^{2}=3,4,5-(\mathrm{MeO})_{3}-\mathrm{C}_{6} \mathrm{H}_{2}(41 \%)$

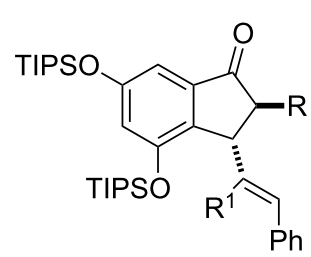

137

136a $\mathrm{R}=\mathrm{Cl}(82 \%$, cis $/$ trans $=5: 4) \quad 137 \mathrm{a} \mathrm{R}=\mathrm{C}(\mathrm{O}) \mathrm{OMe}, \mathrm{R}^{1}=\mathrm{Me}(80 \%) E / Z=19: 1$

136b $\mathrm{R}=\mathrm{Me}(71 \%$, cis $/$ trans $=5: 4) \quad$ 137b $\mathrm{R}=\mathrm{C}(\mathrm{O}) \mathrm{N}(\mathrm{Me})_{2}, \mathrm{R}^{1}=\mathrm{Me}(92 \%) E / Z=19: 1$

137c $\mathrm{R}=\mathrm{S}(\mathrm{O})_{2}-\left(4-\mathrm{Me}^{-\mathrm{C}_{6}} \mathrm{H}_{4}\right), \mathrm{R}^{1}=\mathrm{Me}(85 \%) E / Z=19: 1$

137d $\mathrm{R}=\mathrm{P}(\mathrm{O})(\mathrm{OEt})_{2}(90 \%), \mathrm{R}^{1}=\mathrm{Me} E / Z=19: 1$

TMP: trimethoxyphenyl 137e $\mathrm{R}=\mathrm{C}(\mathrm{O}) \mathrm{OMe}(83 \%), \mathrm{R}^{1}=\mathrm{H} E / Z=1: 19$

Figure 3: Chemical structures of investigated dienones 133 and synthesized cyclic products 134-137.<smiles>[R]C(=C[PH2+])C1c2c(O[AsH3])cc(O[AsH2])cc2C(=O)C1[R]</smiles>

138<smiles>[R]C1C(=O)c2cc3c(cc2C1/C(C)=C/O)OCO3</smiles>

139<smiles>CCC[C@H]1c2[nH]ccc2C(=O)C1C(=O)OC</smiles>

$140(80 \%)$<smiles>CCOP(=O)(OCC)C1C(=O)c2cc[nH]c2[C@H]1C1CCC1</smiles>

$141(71 \%)$<smiles>COC(=O)C1C(=O)c2ccoc2C1/C=C/c1ccccc1</smiles>

$142(71 \%)$

138a $\mathrm{R}=\mathrm{P}(\mathrm{O})(\mathrm{OEt})_{2}, \mathrm{R}^{1}=\mathrm{Me}(76 \%)$

138b $\mathrm{R}=\mathrm{CN}, \mathrm{R}^{1}=\mathrm{Me}(60 \%)$

139a $R=P(O)(O E t)_{2}(97 \%)$

139b $R=$ CN $(59 \%)$

138c $\mathrm{R}=\mathrm{NO}_{2}, \mathrm{R}^{1}=\mathrm{H}(99 \%)$

TIPS: triisopropylsilyl

Figure 4: Chemical structures of 1-indanones and their heteroatom analogues 138-142. 
strates substituted by electron-withdrawing groups, such as $\mathrm{CO}_{2} \mathrm{Me}, \mathrm{P}(\mathrm{O})(\mathrm{OEt})_{2}, \mathrm{CN}$ or $\mathrm{NO}_{2}$. This reaction was carried out in the presence of an iridium catalyst and antimony hexafluoride $\left(\mathrm{AgSbF}_{6}\right)$ under mild conditions. The starting chalcones were almost completely converted into 1-indanones 138-142 and isolated in very good yields.

Our research group synthesized 3-aryl-1-indanones 148 and previously unknown 3-aryl-2-phosphoryl-1-indanones 147 which exhibited anticancer activity against HeLa and K562 cell lines at the $\mu \mathrm{M}$ level [73]. Both groups of products have been obtained from the corresponding phosphorylated chalcones $(Z)$ 145 or nonphosphorylated chalcones $(E)-\mathbf{1 4 6}$, in selective Horner-Wittig or Knoevenagel olefinations, followed by a
Nazarov cyclization using $\mathrm{FeCl}_{3}$ or $\mathrm{AlCl}_{3}$. The 2-phosphorylated chalcones (Z)-145 and non-phosphorylated ones (E)-146 could be obtained from the same substrates, $\beta$-ketophosphonates 143 and aromatic aldehydes 144 depending on the reaction conditions used (piperidine/toluene for the Knoevenagel reaction or NaH/THF for the Horner-Witting reaction) (Scheme 43).

Photochemical reactions play an important role in the synthesis of 1-indanone derivatives. Thus, photolysis of the ketone 149 gave the 1-indanone $\mathbf{1 5 0}$ in $94 \%$ yield. It is worth mentioning that photolysis of the ketone 151a did not lead to the formation of 1-indanone $\mathbf{1 5 2}$ corresponding to $\mathbf{1 5 0}$ but led to the derivative 153a (Scheme 44) [74].

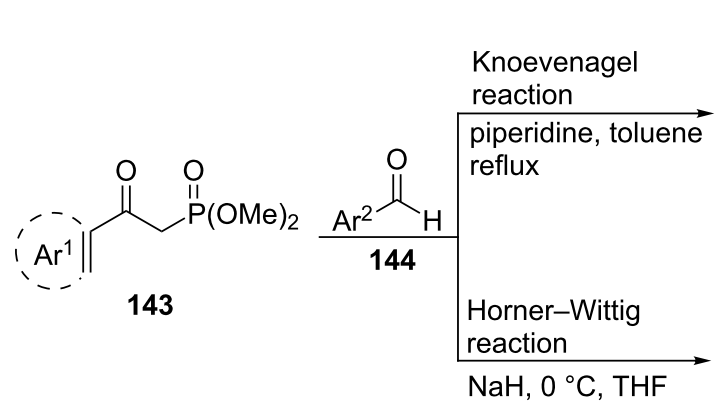

$\mathrm{NaH}, 0{ }^{\circ} \mathrm{C}$, THF<smiles>COP(=O)(OC)C(=C[181I])C(=O)C1=CC=CCC1</smiles>

(Z)-145

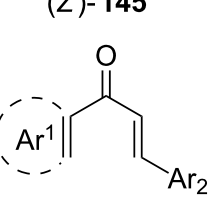

$(E)-146$<smiles>COP(=O)(O)C1C(=O)C2CCCCC2C1[Te]</smiles>

$147 a-c$

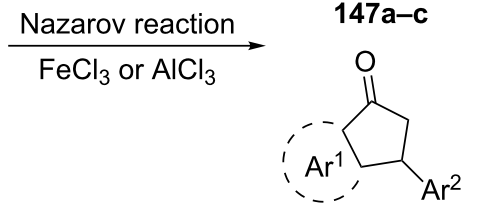

$148 a, b$
$147 a-c$<smiles>[Y]c1cc2c(cc1C)OC(=[Al])O2</smiles>

147a $\mathrm{Ar}^{2}=4-\mathrm{BrC}_{6} \mathrm{H}_{4}(30 \%)$

147b $\mathrm{Ar}^{2}=\mathrm{C}_{6} \mathrm{H}_{5}(80 \%)$

$147 \mathrm{c} \mathrm{Ar}^{2}=4-\mathrm{MeO}(\mathrm{O}) \mathrm{C}-\mathrm{C}_{6} \mathrm{H}_{4}(62 \%)$

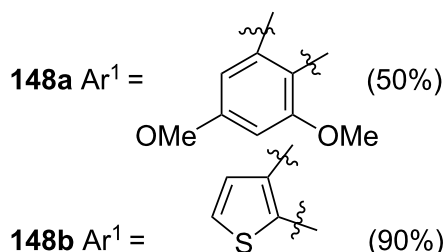

$(90 \%)$<smiles>Cc1ccccc1C(=O)C1=CCCC1</smiles>
$30 \mathrm{~min}$ 149 $150(94 \%)$<smiles>O=C(C1=CCCCC1)c1ccccc1</smiles><smiles>O=C1c2ccccc2C2CCCCC12</smiles>

153b (65\%)<smiles>Cc1ccccc1C(=O)C1=CCCCC1</smiles>

151a<smiles>O=C1c2ccccc2CC12CCCCC2</smiles>

152<smiles>CCCCCCCCCCCC</smiles><smiles>Cc1cccc2c1C(=O)C1CCCCC21</smiles>

153a (57\%) 
The Nazarov-type cyclization has been proposed for the synthesis of polysubstituted-1-indanones $\mathbf{1 5 5} \mathbf{a}-\mathbf{m}$, and $\mathbf{1 5 7} \mathbf{a}-\mathbf{l}$ [75]. They were obtained from 1,4-enediones $\mathbf{1 5 4}$ and aryl vinyl $\beta$-ketoesters $\mathbf{1 5 6}$ in the presence of $\mathrm{AlCl}_{3}$ as a promoter, in high yields (up to $99 \%$ ) (Scheme 45). It was further proved that the pattern of substituents at C-2, C-4 and C-5 positions was essential for the reaction efficiency.

\subsection{From alcohols}

An interesting synthesis of optically active 1-indanones $159 \mathbf{a}-\mathbf{g}$ by a rhodium-catalyzed isomerization of racemic $\alpha$-arylpropargyl alcohols $\mathbf{1 5 8}$ has been developed by Shintani, Okamoto and Hayashi (Scheme 46) [76]. By the mechanistic investigations using deuterium-labeled substrates, the authors have disclosed that the methine proton of the alcohol goes to the $\beta$-position of the 1-indanone, while the ortho-proton of the phenyl group is shifted to the $\alpha$-position.

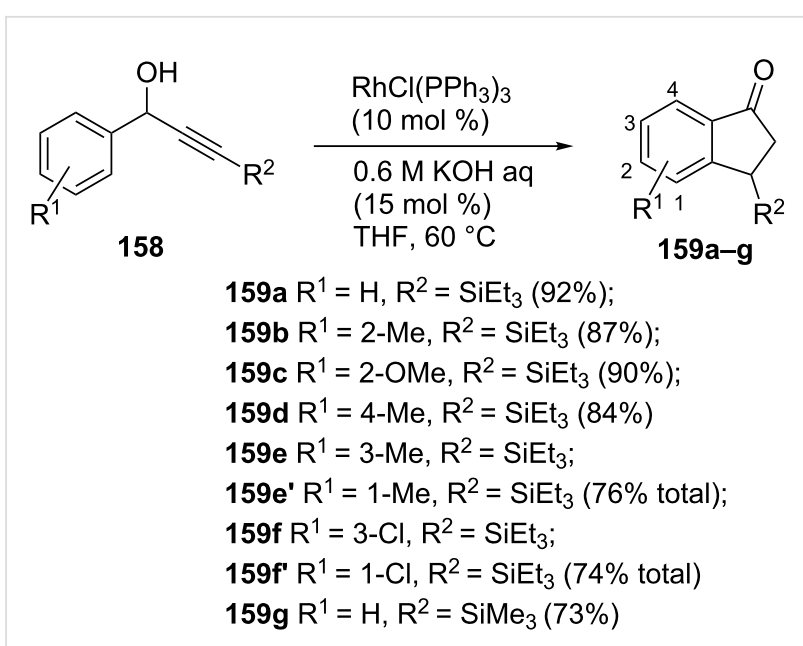

Scheme 46: Synthesis of 1-indanones $159 a-g$ from a-arylpropargyl alcohols 158 using $\mathrm{RhCl}\left(\mathrm{PPh}_{3}\right)_{3}$ as a catalyst.<smiles>CCOC(=O)C(=CC(=O)Br)C(=O)c1cc(OC)c(OC)c(OC)c1</smiles>

$154 a-m(E / Z$ isomer $)$<smiles>CCOC(=O)C1C(=O)c2cc(OC)c(OC)c(OC)c2[C@H]1C(=O)Br</smiles>

155a-m

155a $\mathrm{Ar}=\mathrm{C}_{6} \mathrm{H}_{5}(96 \%)$

155b Ar $=4-\mathrm{MeC}_{6} \mathrm{H}_{4}(95 \%)$

$155 \mathrm{c} \mathrm{Ar}=\mathrm{MeOC}_{6} \mathrm{H}_{4}(99 \%)$

155d Ar $=3,4$-(ethylene-1,3-dioxa) $\mathrm{C}_{6} \mathrm{H}_{3}(99 \%)$

155e $\mathrm{Ar}=4-\mathrm{ClC}_{6} \mathrm{H}_{4}(94 \%)$

$155 f \mathrm{Ar}=4-\mathrm{BrC}_{6} \mathrm{H}_{4}(89 \%)$

155g Ar $=4-\mathrm{NO}_{2} \mathrm{C}_{6} \mathrm{H}_{4}(85 \%)$

155h $\mathrm{Ar}=3,4-\mathrm{Cl}_{2} \mathrm{C}_{6} \mathrm{H}_{3}(99 \%)$

155i $\mathrm{Ar}=2$-furyl $(95 \%)$

155j Ar $=2$-thienyl $(92 \%)$

155k Ar $=2$-benzofuryl $(90 \%)$

155I Ar $=2$-naphthyl $(96 \%)$

155m Ar = 1-naphthyl (94\%)<smiles>[R]C=C([R])C(=O)c1cc#[R]cc1</smiles>

156a-I (E/Z isomer)<smiles>[R]C(=O)[C@H]1c2c(cc(OC)c(OC)c2[R])C(=O)[C@H]1C(=O)OCC</smiles>

$157 a-e$

157a $R^{1}=P h, R^{2}=H(92 \%)$

157b R $\mathrm{R}^{1}=4-\mathrm{MeOC}_{6} \mathrm{H}_{4}, \mathrm{R}^{2}=\mathrm{H}(90 \%)$

157c $\mathrm{R}^{1}=4-\mathrm{NO}_{2} \mathrm{C}_{6} \mathrm{H}_{4}, \mathrm{R}^{2}=\mathrm{H}(88 \%)$

$157 d R^{1}=$ OEt, $R^{2}=$ OMe $(87 \%)$

157e $R^{1}=$ OEt, $R^{2}=H(85 \%)$<smiles>CCOC(=O)[C@H]1C(=O)c2cc(OC)c(OC)c(OC)c2[C@H]1C(=O)/C=C/c1ccc(OC)cc1</smiles>

$157 f(93 \%)$<smiles>[R]c1ccc([C@H]2c3cc([R])c([R])c([R])c3C(=O)C2C(=O)OCC)cc1</smiles>

$157 \mathrm{~g}-\mathrm{I}$

$157 g R^{1}=R^{2}=R^{3}=R^{4}=H(90 \%)$

$157 h R^{1}=R^{2}=R^{3}=H, R^{4}=\operatorname{Me}(95 \%)$

157i $R^{1}=R^{2}=R^{3}=H, R^{4}=$ OMe $(96 \%)$

157j $R^{1}=R^{2}=R^{4}=H, R^{3}=$ OMe $(93 \%)$

157k $R^{1}=O M e, R^{2}=R^{3}=R^{4}=H(90 \%)$

$1571 R^{1}=R^{4}=H, R^{2}=R^{3}=$ OMe $(92 \%)$ 
The same research group has proposed another asymmetric isomerization of racemic alcohols $\mathbf{1 6 1}$ leading to the formation of 1 -indanones 162 [77]. In this reaction, $\beta$-chiral 1-indanones 162 were obtained by isomerization of racemic $\alpha$-arylpropargyl alcohols 161 in the presence of a rhodium catalyst. A high enan- tioselectivity has been achieved by the use of the chiral bisphosphine ligand $(R, R)-\mathbf{1 6 0}$ (Scheme 47). A catalytic cycle of this isomerization is shown in Scheme 48. First, alkoxorhodium 163, next alkenylrhodium 165 were formed as intermediates as a result of dehydration and $\beta-\mathrm{H}$ elimination followed by

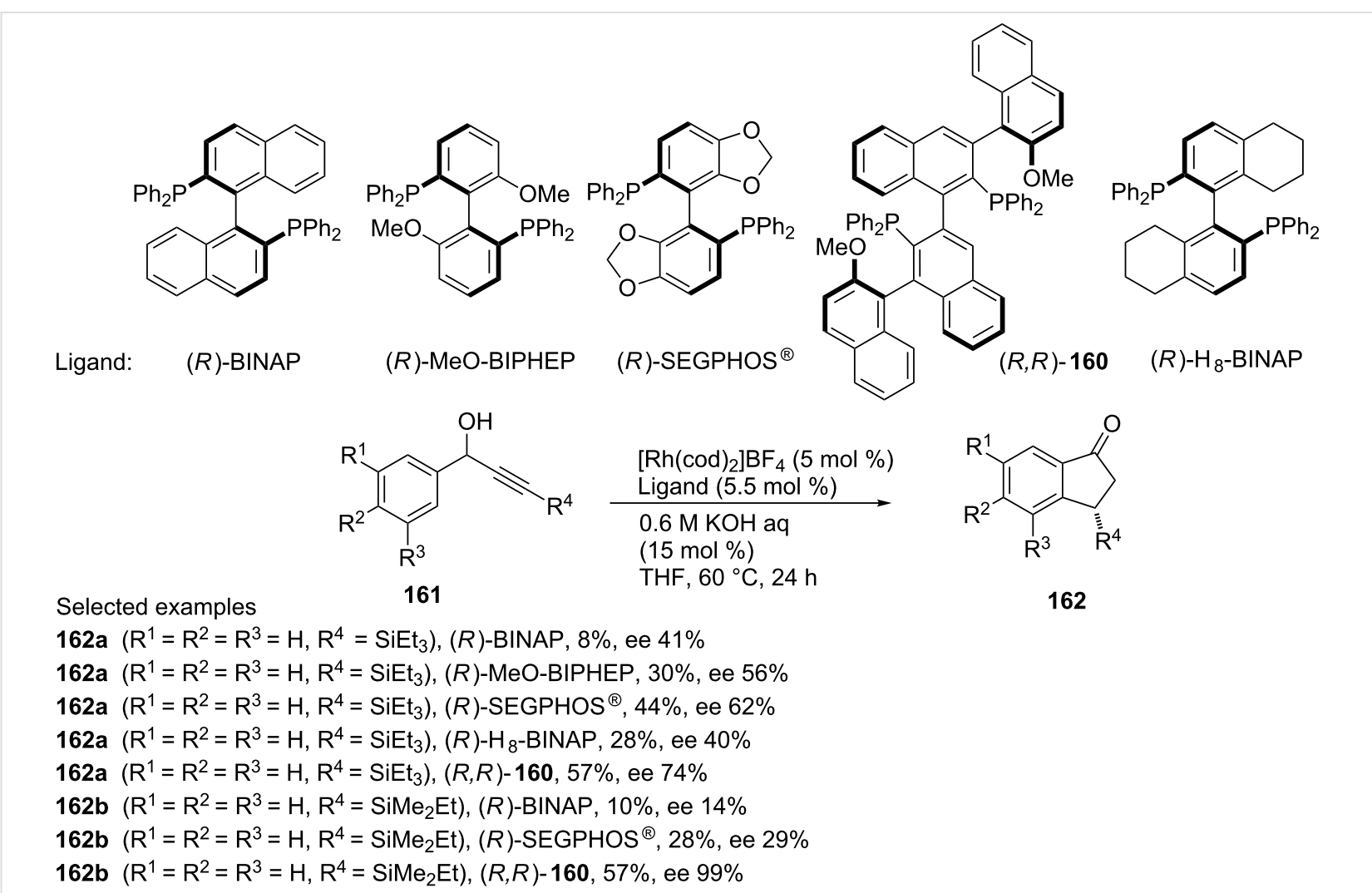

Scheme 47: Synthesis of optically active 1-indanones 162 via the asymmetric Rh-catalyzed isomerization of racemic alcohols 161 using optically pure bisphosphine ligands and $\mathrm{Rh}(\mathrm{cod})_{2} \mathrm{BF}_{4}$ as a catalyst.

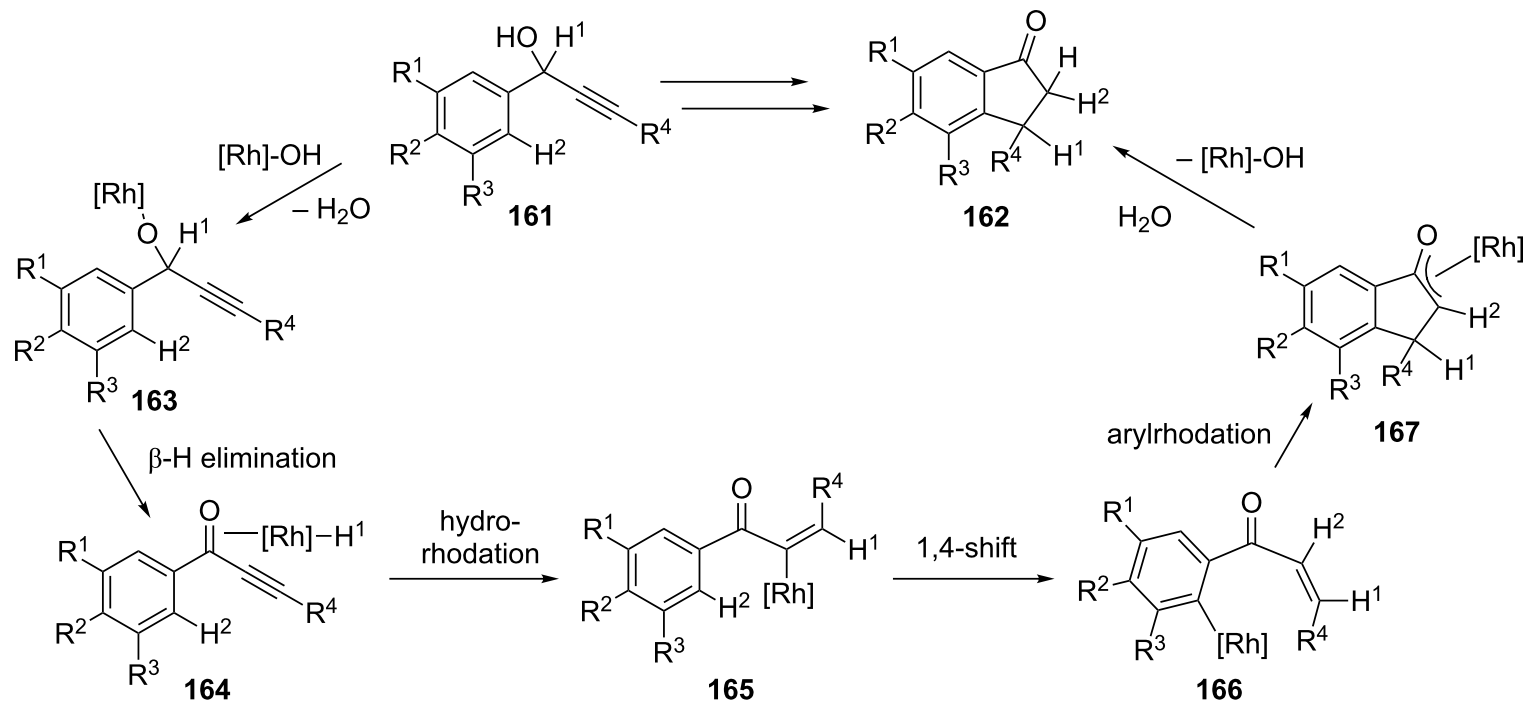

Scheme 48: Mechanism of the Rh-catalyzed isomerization of $\alpha$-arylpropargyl alcohols 161 to 1-indanones 162. 
hydrorhodation, respectively. Then, rhodium 1,4-migration, alkylrhodation and finally rhodium elimination led to 1-indanones 162 via intermediates 166 and 167.

Abicoviromycin (168) is an antiviral and antifungal molecule produced by bacteria. Because of its interesting biological activity, Mitchell and Liebeskind have decided to synthesize abicoviromycin (168) derivatives [78]. In spite of the potent biological activity, abicoviromycin (168) is extremely heat- and acid-sensitive. Moreover, this compound polymerizes rapidly even at low temperatures, such as $-50{ }^{\circ} \mathrm{C}$. Therefore, until 1989 abicoviromycin (168) has not been successfully synthesized. The unit which probably determines the reactivity and unstability of abicoviromycin (168) is the diene-imine fragment. Due this fact, the authors have decided to replace the double bond at the 6,7 position by the benzene ring in the new abicoviromycin derivative $\mathbf{1 6 9}$ to increase the stability while still retaining the biological activity (Figure 5). Thus, the palladium-catalyzed ring expansion of 2-alkynyl-2-hydroxybenzocyclobutenone $\mathbf{1 7 0}$ allowed to obtain alkylidenoindanedione intermediate $\mathbf{1 7 1}$, which was further converted into racemic benzoabicoviromycin 172 (Scheme 49). The racemic benzoabicoviromycin
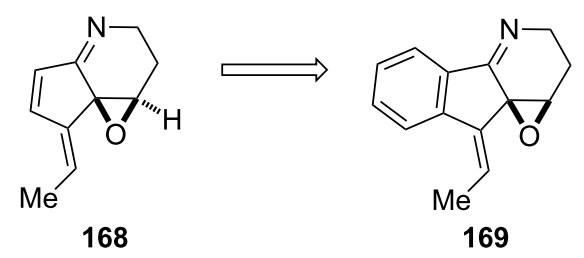

Figure 5: Chemical structure of abicoviromycin (168) and its new benzo derivative 169 .
172 as well as its ( $Z$ )-ethylidene stereoisomer have been screened for in vitro biological activity (antiviral, anticancer and antifungal). The significant in vitro cytotoxicity was observed against the following cell lines: A549 ( $\mathrm{IC}_{50}: 5.48-5.01 \mathrm{mg} / \mathrm{mL}$ ), A549/VP ( IC $\left._{50}: 4.76-4.18 \mathrm{mg} / \mathrm{mL}\right)$, B16-PRIM (IC $50: 0.16 \mathrm{mg}$ / $\mathrm{mL}$ ), HCT116 ( $\left.\mathrm{IC}_{50}: 1.47-141 \mathrm{mg} / \mathrm{mL}\right), \mathrm{HCT} / \mathrm{VP} 35$ ( $\mathrm{IC}_{50}$ : $1.26-1.16 \mathrm{mg} / \mathrm{mL})$. Unfortunately, these levels of activity turned out to be useless in vivo. However, considering the enormous potential of abicoviromycin, syntheses of its additional analogs are reasonable.

\subsection{From alkyl chlorides}

Radiolabeled tracers can supply precious information about structures of biocatalytic reaction networks. The $\left[{ }^{14} \mathrm{C}\right] 1-$ indanone $\mathbf{1 7 5}$ has been used in the synthesis of the $\left[{ }^{14} \mathrm{C}\right]$ indene 176, which was next applied for the examination of the indene bioconversion network expressed in Rhodococcus sp. KY1 [79]. The $\left[{ }^{14} \mathrm{C}\right] 1$-indanone $\mathbf{1 7 5}$ was obtained in the one-pot synthesis involving the Friedel-Crafts acylation of $\left[{ }^{14} \mathrm{C}\right]$ benzene 173 with chloropropionic acid chloride $\mathbf{1 7 4}$ followed by a Friedel-Crafts cyclization in the presence of concentrated $\mathrm{H}_{2} \mathrm{SO}_{4}$. The $\left[{ }^{14} \mathrm{C}\right] 1-$ indanone 175 was then converted in three steps to $\left[{ }^{14} \mathrm{C}\right]$ indene 176 (Scheme 50).

The same reaction sequence involving the Friedel-Crafts acylation of disubstituted benzene derivatives 177 with 3-chloropropionyl chloride $\mathbf{1 7 4}$ followed by a intramolecular Friedel-Crafts alkylation afforded 1-indanones 178 (Scheme 51) [80]. A direct reaction of the latter with $n$-butylnitrite led to the formation of keto-oximes 179 which underwent a $\mathrm{Pd} / \mathrm{C}$ catalytic reduction to give 2-amino substituted 1-indanones $\mathbf{1 8 0}$. Both keto-oximes 179 and 2 -amino derivatives 180 are $\beta_{2}$-adrenergic agonists tested for bronchodilating activity.

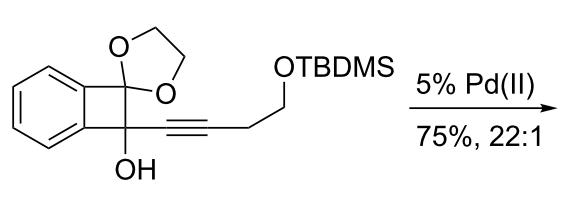

170<smiles></smiles>

171<smiles>C=C1c2ccccc2C2=NCCC3OC123</smiles>

$172 E / Z(1: 1)$

Scheme 49: Synthesis of racemic benzoabicoviromycin 172.

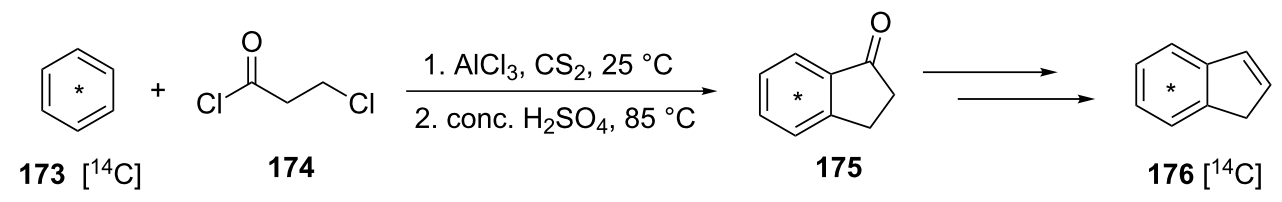


<smiles>[R]c1ccccc1[R]</smiles>

177<smiles>CC(=O)CCCl</smiles>

174<smiles>[R]c1cc2c(cc1[R])C(=O)CC2</smiles>

178

$$
178 \underset{\mathrm{MeOH}, \text { conc. } \mathrm{HCl}, 2 \mathrm{~h}, 40^{\circ} \mathrm{C},}{\stackrel{1.1 \text { equiv } n \text {-BuONO, } 25: 1}{\longrightarrow}}
$$

$(54-93 \%)$<smiles>[R]c1cc2c(cc1[R])C(=O)/C(=N/O)C2</smiles>

179

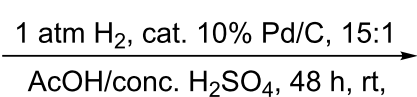

$(11-88 \%)$<smiles>[R]c1cc2c(cc1[R])C(=O)C(N)C2</smiles>

180

$180 \mathrm{a} \mathrm{R}=\mathrm{H} \quad 180 \mathrm{e} \mathrm{R}=n-\mathrm{Bu}$

$180 \mathrm{~b} R=\mathrm{Me} 180 \mathrm{f} R=\mathrm{OMe}$

$180 \mathrm{c} R=\mathrm{Et} \quad 180 \mathrm{~g} R=\mathrm{H}$

$180 \mathrm{~d} R=n-\operatorname{Pr}$

Scheme 51: Synthesis of indanone derivatives 178-180.

The pterosin family are sesquiterpenoids naturally occurring in bracken fern (Pteridium aquilinum), some of them exhibit antibacterial and cytotoxic activity. A practical synthesis of pterosin A (186), being a 1-indanone derivative, has been proposed by Uang et al. [81]. In this synthesis, 3-chloropropionyl chloride (174) reacted with 2-bromo-1,3-dimethylbenzene (181) in the presence of $\mathrm{AlCl}_{3}$ to give two isomeric products 182 and $\mathbf{1 8 3}$. The mixture of 182 and $\mathbf{1 8 3}$ was heated with concentrated $\mathrm{H}_{2} \mathrm{SO}_{4}$ at $90{ }^{\circ} \mathrm{C}$ to form the corresponding 1-indanones $\mathbf{1 8 4}$ and
185 (in 39\% and 40\% yield, respectively). The 1-indanone $\mathbf{1 8 4}$ was converted to pterosin A (186) in a sequence of reactions (Scheme 52).

\subsection{From alkynes}

The use of a catalytic amount of antimony pentafluoride and ethanol converted mixtures of phenylalkynes 187 and aldehydes 188 to trans-2,3-disubstituted 1-indanones $\mathbf{1 8 9}$ in the one-pot reaction (Scheme 53) [82].<smiles>O=C(Cl)CCCl</smiles>

174<smiles>Cc1cccc(C)c1Br</smiles>

181

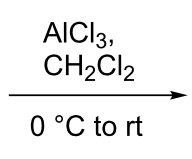$$
\mathrm{Me}
$$<smiles>Cc1cc(C(=O)CCCl)cc(C)c1Br</smiles>

$\underset{90{ }^{\circ} \mathrm{C}, 1 \mathrm{~h}}{\stackrel{\text { conc. } \mathrm{H}_{2} \mathrm{SO}_{4}}{\longrightarrow}}$<smiles>Cc1cc2c(c(C)c1Br)C(=O)CC2</smiles>

$184(99 \%)$<smiles>[V]=[Tl]</smiles><smiles>Cc1cc2c(c(C)c1CCO)C(=O)C(C)(CO)C2</smiles>

$186(85 \%)$ 
<smiles>IC#Cc1ccccc1</smiles>

187<smiles>[R]C=[O+]</smiles>

188

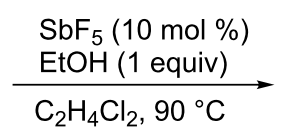<smiles>[R]C1C(=O)c2ccccc2C1[R]</smiles>

189

189a-e $\mathrm{R}^{1}=\mathrm{Me}, 189 \mathrm{a} \mathrm{R}^{2}=\mathrm{Ph}(78 \%) ; 189 \mathrm{~b} \mathrm{R}^{2}=\mathrm{PhCH}(\mathrm{OEt})_{3}(55 \%) ; 189 \mathrm{c} \mathrm{R} \mathrm{R}^{2}=t-\mathrm{Bu}(89 \%) ;$

189d R $R^{2}=$ Et $(75 \%) ; 189 e R^{2}=P h(72 \%)$

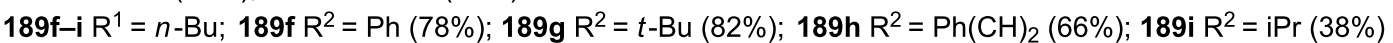

189j,k R ${ }^{1}=\mathrm{Ph} ; \mathbf{1 8 9 j} \mathrm{R}^{2}=\mathrm{Ph}(59 \%) ; 189 k R^{2}=\mathrm{Me}(45 \%)$

189I $R^{1}=H, R^{2}=P h(59 \%)$

Scheme 53: Synthesis of trans-2,3-disubstituted 1-indanones 189

A synthesis of 3-aryl-1-indanone derivatives 192 from aromatic aldehydes 190 and alkyne derivatives 191 has been patented by $\mathrm{Xi}$ and Liu in 2016 [83]. The reaction proceeded in the presence of methyl trifluoromethanesulfonate, in dichloromethane or 1,2-dichloroethane in high yields (Scheme 54).

\subsection{From nitriles}

Nitrile derivatives are also useful substrates for the synthesis of 1-indanones. An efficient synthesis of 1-indanones 194 via palladium-catalyzed cyclization of 3-(2-iodoaryl)propanenitriles 193 has been described by Pletnev and Larock [84]. This reaction was compatible with a wide variety of electron-donor and electron-acceptor functional groups. The authors have also found that the formation of 1-indanones 194 is accompanied by the reduction of the carbon-iodine bond in 3-(2-iodoaryl)propanonitriles 193 leading to the formation of the nitrile 195 as a byproduct (Scheme 55).

Cyclization of 3-phenylnaphtalene-2-carbonitrile (199) in the presence of polyphosphoric acid gave 1-indanone $\mathbf{2 0 0}$ in $76 \%$ yield. The nitrile 199 was obtained from benzaldehyde 196 as a result of sequencial reactions leading to intermediates 197 and 198 [85]. The authors have also synthesized other fluorenone derivatives 200-204 by using this method (Scheme 56).

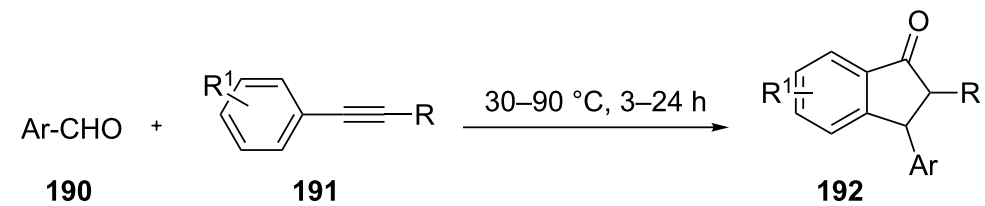

$\mathrm{Ar}=$ substituted phenyl or substituted naphthyl; $\mathrm{R}=$ aryl or $\mathrm{C}_{1-4}$ alkyl; $\mathrm{R}^{1}=\mathrm{Me}, \mathrm{Et}, \mathrm{OMe}$

Scheme 54: Synthesis of 3-aryl-1-indanone derivatives 192<smiles>[R]c1cc(I)c(C([R])C([R])(C)N)cc1[R]</smiles>

193<smiles>CCOCCO</smiles>

$\mathrm{DMF} / \mathrm{H}_{2} \mathrm{O}$<smiles>[R]c1cc2c(cc1[R])C([R])C([R])([R])C2=O</smiles>

194<smiles>[R]c1ccc(C([R])C([R])(C)C)cc1[R]</smiles>

195

193a $R^{1}=R^{2}=H, R^{3}=R^{4}=M e, R^{5}=H ; 194 a(88 \%) ; 195 a(12 \%)$ 193b $R^{1}=R^{2}=H, R^{3}=R^{4}=-\left(C_{2}\right)_{5^{-}}, R^{5}=H ; 194 b(86 \%) ; 195 b(8 \%)$

193c $R^{1}=R^{2}=H, R^{3}=R^{4}=P h, R^{5}=H ; 194 c(92 \%) ; 195 c(7 \%)$ 193d $R^{1}=R^{2}=H, R^{3}=H, R^{4}=E t, R^{5}=H ; 194 d(86 \%) ; 195 d(8 \%)$ 193e $R^{1}=R^{2}=H, R^{3}=M e, R^{4}=E t, R^{5}=C_{2} M e ; 194 e(78 \%) ; 195 e$ (traces) 193f $R^{1}=R^{2}=$ OMe, $R^{3}=R^{4}=M e, R^{5}=H ; 194 f(75 \%) ; 195 f$ (traces) 


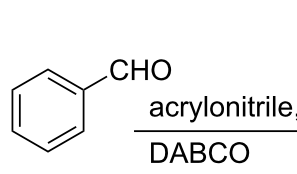

196<smiles>C=C(C#N)C(O)c1ccccc1</smiles>

197
1. $\mathrm{HBr}$

2. In, 2-bromo benzaldehyde

3. $\mathrm{Ac}_{2} \mathrm{O}$<smiles>O=C1c2ccccc2-c2cc3ccccc3cc21</smiles>

$200(76 \%)$

199

PPA, $120^{\circ} \mathrm{C}$<smiles>O=C1c2cc3ccccc3cc2-c2ccc3ccccc3c21</smiles>

$203(69 \%)$<smiles>O=C1c2ccccc2-c2cc3ccc4ccccc4c3cc21</smiles>

$202(73 \%)$<smiles>C=C(C#N)C(c1ccccc1)C(OC(C)=O)c1ccccc1Br</smiles>

198 (syn/anti)<smiles>Cc1ccc2c(c1)C(=O)c1cc3ccccc3cc1-2</smiles>

$201(75 \%)$<smiles>CC(C)(C)OC(=O)OCc1ccccc1</smiles>

$204(64 \%)$

Scheme 56: Synthesis of 1 -indanones $\mathbf{2 0 0 - 2 0 4}$ by cyclization of aromatic nitriles.

\subsection{From diazo compounds}

Hashimoto et al. have proposed the synthesis of optically active 1,1 '-spirobi[indan-3,3'-dione] derivative $\mathbf{2 0 8}$ (up to $80 \%$ enantiomeric excess) from bis( $\alpha$-diazo- $\beta$-keto ester) 205 [86]. The key step of this synthesis was a double intramolecular $\mathrm{C}-\mathrm{H}$ insertion process catalyzed by dirhodium(II) tetrakis $[N$ phthaloyl-( $R$ or $S)$-tert-leucinate]. The resulting spiroindanone derivative 207 obtained from the intermediate 206, underwent demethoxycarbonylation to give 1,1'-spirobi[indan-3,3'-dione] 208 (Scheme 57).

Atipamezole is a synthetic $\alpha_{2}$-adrenergic receptor antagonist used in veterinary for reversal of the sedative and analgesic effects induced by $\alpha_{2}$-adrenergic receptor agonists. Vacher et al. synthesized $\alpha_{2}$-adrenergic receptor antagonists, potentially more selective than known compounds [87]. Transformation of diazo compounds 209 into 1-indanone derivatives 210 , catalyzed by rhodium acetate, has been one of the steps in the total synthesis of atipamezole analogues 211 (Scheme 58).

The most common symptom of the menopause is hot flash, which is characterized by sweating, sudden feeling of heat, palpitation or anxiety. Hormone replacement therapy (HRT) alleviates above mentioned symptoms but its use has been limited because of many side effects, such as increased hormone-dependent cancers risk. Watanabe et al. have synthesized a selective estrogen receptor modulator, 3-[4-(1-piperidinoethoxy)phenyl] spiro[indene-1,1'-indane]-5,5' -diol hydrochloride (216) which may be used for a new treatment of hot flush [88]. In this synthesis, the reaction of 5-methoxyindan-1one (212) with the Grignard reagent 217 followed by acid-catalyzed dehydration and hydrogenolysis of the resulting double bond with $\mathrm{Pd}(\mathrm{OH})_{2} / \mathrm{C}$, gave benzoic acid 213. Next, the latter was converted to $\alpha$-diazo- $\beta$-keto ester 214 which then was

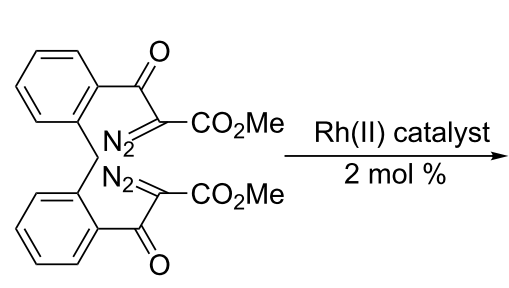

205

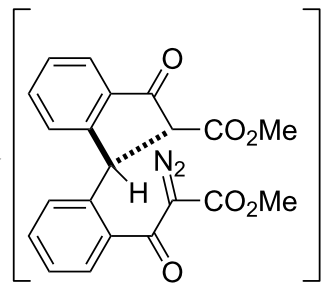

206

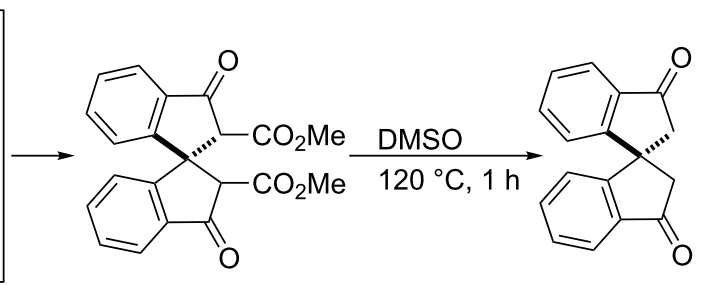

207
208

Scheme 57: Synthesis of 1,1'-spirobi[indan-3,3'-dione] derivative 208. 
<smiles>[R]C([R])=C([R])c1ccccc1C(=O)C(=[W])CCC(=O)OCC</smiles>

209<smiles>[R]C1([R])C2([R])C(=O)c3ccccc3C1([R])C2([R])COCC</smiles>

211 210a $R^{1}=R^{2}=R^{3}=H(76 \%)$

210b-exo $R^{1}=M e, R^{2}=R^{3}=H(45 \%)$

210c-endo $R^{1}=R^{3}=H, R^{2}=M e(44 \%)$

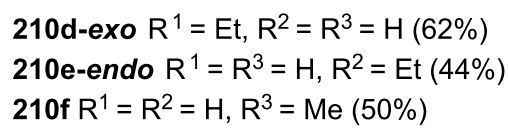

Scheme 58: Total synthesis of atipamezole analogues 211.

submitted to the rhodium(II) acetate catalyzed intramolecular, carbon-hydrogen insertion reaction to give the spiroindane 215 . Finally, the spiroindane $\mathbf{2 1 5}$ was converted to the expected, estrogen receptor modulator 216 (Scheme 59).

3-Arylindan-1-ones 219, versatile intermediates for the synthesis of a number of biologically active compounds, have been synthesized from $\alpha$-diazo- $\beta$-keto ester 218 via a intramolecular $\mathrm{C}-\mathrm{H}$ insertion reaction catalyzed by the rhodium(II) complex 220 followed by the carboxylic methyl ester hydrolysis/ decarboxylation in $\mathrm{DMSO} / \mathrm{H}_{2} \mathrm{O}$ at $120{ }^{\circ} \mathrm{C}$ with up to $72 \%$ enantiomeric excess (Scheme 60) [89].

\subsection{From epoxides and cyclopropanes}

The chalcone epoxides 221 ring opening catalyzed by indium(III) chloride, followed by a intramolecular Friedel-Crafts alkylation has been used by Ahmed et al. for the synthesis of 2-hydroxyindan-1-one derivatives 222 in good yields (Scheme 61) [90].

The same research group used the THP (tetrahydropyranyl) and MOM (methoxymethyl) protected chalcone epoxides and tin(IV) chloride under mild conditions to synthesize dihydroxy substituted 1-indanones [91]. All reactions have been completed within 2-3 $\mathrm{min}$ at $0{ }^{\circ} \mathrm{C}$ in the presence of the catalyst $\mathrm{Sn}(\mathrm{IV}) \mathrm{Cl}_{4}$ (225) and gave products in excellent yields (90-98\%). For example, 1-indanone derivative 224 has been obtained from the THP/MOM protected chalcone epoxide $\mathbf{2 2 3}$ in $98 \%$ yield for both THP and MOM ethers (Scheme 62).

Irradiation of aromatic $\gamma, \delta$-epoxy ketones $\mathbf{2 2 6}$ with a mediumpressure UV mercury lamp $(450 \mathrm{~W})$ led to the formation of 1-indanones 227 via a photochemical epoxy rearrangement and 1,5-biradical cyclization tandem reaction (Scheme 63) [92]. The<smiles>CCOC(=O)C(=N)c1cc(OC)ccc1C1CCc2cc(OC)ccc2C1C(=O)OCC</smiles> 
<smiles>CC(=O)C(=N)C(=O)c1ccccc1Cc1ccccc1</smiles>

218

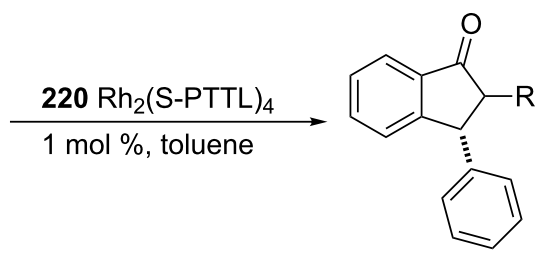

219

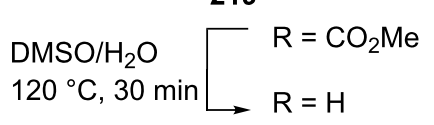<smiles>[R]c1ccc(C(=O)C2OC2c2ccc([R])cc2)cc1</smiles>

221

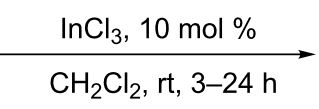

$\mathrm{CH}_{2} \mathrm{Cl}_{2}, \mathrm{rt}, 3-24 \mathrm{~h}$<smiles>[R]c1ccc(C2c3cc([R])ccc3C(=O)C2O)cc1</smiles>

222

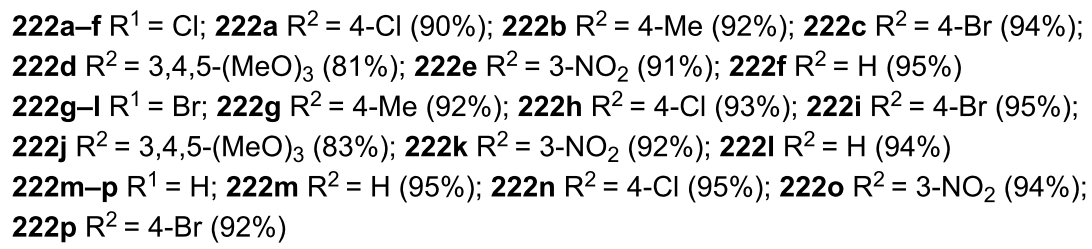

Scheme 61: Synthesis of 2-hydroxy-1-indanones 222.<smiles>[R20]c1ccc(C(=O)C2OC2c2ccc(Cl)cc2)cc1</smiles>

$223 \mathrm{R}^{1}=\mathrm{MOM} / \mathrm{THP}$<smiles>O=C1c2ccc(O)cc2C(c2ccc(Cl)cc2)C1O</smiles>

$224(98 \%)$
Scheme 62: Synthesis of the 1-indanone 224 from the THP/MOM protected chalcone epoxide 223.

best yields (up to $84 \%$ ) were achieved by using substrates $\mathbf{2 2 6}$ with electron-acceptor substituents at the para position of the aryl group.

A new method for the synthesis of optically active $\alpha$-hydroxy ketones by asymmetric oxidation of the enol phosphates catalyzed by Sharpless reagents or chiral dioxirane has been pro- posed by Krawczyk et al. [93]. For example, optically active 1 -indanone 230 was obtained from the cyclic enol phosphate 228 which next was reacted with a fructose-derived dioxirane 232 generated in situ from the ketone 231, to provide the epoxide 229 (Scheme 64). Then, the latter was hydrolyzed with $\mathrm{CF}_{3} \mathrm{C}(\mathrm{O}) \mathrm{OH}$ in $\mathrm{Et}_{2} \mathrm{O} / \mathrm{H}_{2} \mathrm{O}$ at $0{ }^{\circ} \mathrm{C}$ to obtain optically active 1 -indanone 230.

A very interesting approach for the synthesis of 1-indanones 234 based on the rearrangement of cyclopropanol derivatives 233, has been reported in 2012 by Rosa and Orellana [94]. This reaction was carried out in the presence of palladium catalyst and gaseous oxygen as the terminal oxidant (Scheme 65).

\subsection{From other compounds}

In 2016, Shi et al. have developed an unique, conditions-controlled [ $\left.\mathrm{Rh}_{2}(\mathrm{esp})_{2}\right]$ (esp $=\alpha, \alpha, \alpha^{\prime}, \alpha^{\prime}$-tetramethyl-1,3benzenedipropionic acid)-catalyzed reaction of $N$-sulfonyl1,2,3-triazoles 235 leading to a mixture of 1,2-dihydroisoquino- 
<smiles>[R]C(=O)c1cc([R])c([R])cc1C1OC1([R])[R]</smiles>

226<smiles>[R]c1cc2c(cc1[R])C([R])(O)C([R])([R])C2=O</smiles>

227

227a $R^{1}=R^{2}=H, R^{3}=P h, R^{4}=M e, R^{5}=H(62 \%) ; 227 b R^{1}=R^{2}=H, R^{3}=P h, R^{4}=E t, R^{5}=H(61 \%) ;$

227c $R^{1}=R^{2}=H, R^{3}=4-C_{2} M e-C_{6} H_{4}, R^{4}=M e, R^{5}=H(84 \%) ; 227 d R^{1}=R^{2}=H, R^{3}=P h, R^{4}=R^{5}=M e(80 \%) ;$

227e $R^{1}=R^{5}=H, R^{2}=C l, R^{3}=P h, R^{4}=M e(63 \%) ; 227 f R^{1}=C l, R^{2}=R^{5}=H, R^{3}=4-C_{2} M e-C_{6} H_{4}, R^{4}=M e(66 \%)$

227g $R^{1}=\mathrm{Cl}, \mathrm{R}^{2}=\mathrm{R}^{5}=\mathrm{H}, \mathrm{R}^{3}=\mathrm{Ph}, \mathrm{R}^{4}=\mathrm{Me}(63 \%)$

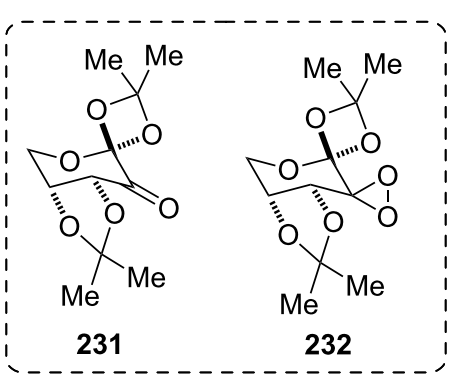<smiles>CCOC(=O)C1=C(C)Cc2ccccc21</smiles>

$(E)$ or $(Z) 228$

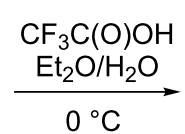

$(\mathrm{EtO})_{2}(\mathrm{O}) \mathrm{PO}$

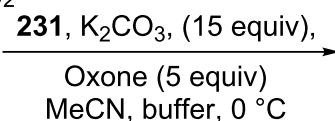
$\mathrm{MeCN}$, buffer, $0{ }^{\circ} \mathrm{C}$

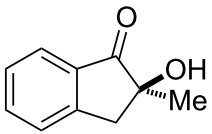

(S)-(-)-230 (25\%)<smiles>[R]c1cc(C2(O[Na])C(C)(C)C2(C)C)c([R])c([R])c1[R]</smiles>

233
0.05 equiv $\mathrm{Pd}(\mathrm{OAc})_{2}$ 2.0 equiv $\mathrm{KOAC}$ 1.1 equiv TBAF $\mathrm{H}_{2} \mathrm{O}$

$\mathrm{O}_{2}$, DMAc, $100^{\circ} \mathrm{C}$<smiles>[R]c1c([R])c([R])c2c(c1[R])CC(C)(C)C2=O</smiles>

234<smiles>CC(=O)N(C)CCI(C)CI</smiles>

234a $R^{1}=R^{2}=R^{4}=H, R^{3}=$ OMe $(75 \%) \quad 234 f R^{1}=R^{2}=R^{4}=H, R^{3}=C(O)$ OMe $(84 \%)$

234b $R^{1}=O M e, R^{2}=R^{3}=R^{4}=H(78 \%)$

234c $R^{1}=R^{3}=R^{4}=H, R^{2}=$ OMe $(70 \%)$

234d $R^{1}=H, R^{2}=R^{4}=R^{3}=$ OMe $(75 \%)$

234e $R^{1}=R^{3}=R^{4}=H, R^{2}=C(O)$ OMe $(88 \%)$

234g $R^{1}=R^{2}=R^{4}=H, R^{3}=C_{3}(84 \%)$<smiles>CC12Cc3ccccc3C1(C)O2</smiles>

$(S, S)-\mathbf{2 2 9}(28 \%)$

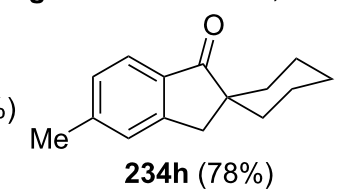

Scheme 65: Synthesis of 1-indanone derivatives 234 from cyclopropanol derivatives 233.

lines 236 and substituted 1-indanone derivatives 237 via alkoxy group migration in 16 and $57 \%$ yields, respectively (Scheme 66) [95].

\section{Construction of the 6-membered ring}

The titles of subsections in this chapter contain names of the 1-indanone precursors which provide the biggest number of carbon atoms during the synthesis of the 1-indanone benzene ring
For instance, 1,3-dienes in the Diels-Alder reaction provide 4 carbon atoms of the six ones needed to construct the benzene ring of 1-indanone compared to dienophiles which deliver only two of them.

\subsection{From 1,3-dienes}

Wolf and Xu have synthesized 7-methyl substituted 1-indanone 241 utilizing 1,3-pentadiene (238) and 2-cyclopentenone (239) 


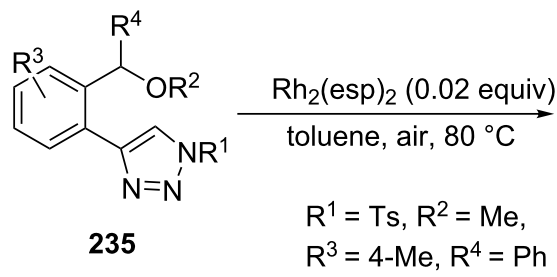<smiles>[R10]C1C=C(O)c2cccnc2C1[R1]</smiles><smiles>[R]C1C(=O)c2ccccc2C1[R]</smiles>

$236(16 \%)$

$237(57 \%)$

Scheme 66: Synthesis of substituted 1-indanone derivatives 237.

as starting compounds [96]. 7-Methyl substituted 1-indanone 241 has been obtained in the Diels-Alder reaction between 1,3pentadiene (238) and 2-cyclopentenone (239) followed by the oxidative aromatization with $\mathrm{Pd} / \mathrm{C}$ (Scheme 67). The latter was further used as a substrate for the synthesis of bisoxazolidine ligand 242. The same Diels-Alder reaction to obtain $\mathbf{2 4 1}$ has been used by Katsumura et al. [97]. In this case, 241 was further converted to cis-1-amino-7-methyl-2-indanol (243, Scheme 67).

Katsumura et al. have also synthesized disubstituted 1-indanone 246 using the Diels-Alder reaction [97]. This synthesis utilized the siloxydiene 244 and 2-cyclopentenone (239) which were reacted in the presence of 2,5-di-tert-butylhydroquinone (DBHQ) in benzene, followed by treatment with $p$-toluenesulfonic acid in acetone to give the diketone $\mathbf{2 4 5}$ (Scheme 68). Then, the latter underwent oxidative aromatization by treatment with $\mathrm{Pd} / \mathrm{C}$ in $p$-cymene. The synthesized 1-indanone 246 was further converted to the cis-1-amino-2-indanol 247 and used as ligand for asymmetric reactions.

A similar way to synthesize 5-hydroxy substituted 1-indanone $\mathbf{2 5 0}$ by utilizing the 1,3-diene $\mathbf{2 4 8}$ and the sulfoxide $\mathbf{2 4 9}$, has been described by Danishefsky et al. [98]. As a result of the cycloaddition, 5-hydroxy-1-indanone (250) has been obtained in $68 \%$ yield (Scheme 69 ).

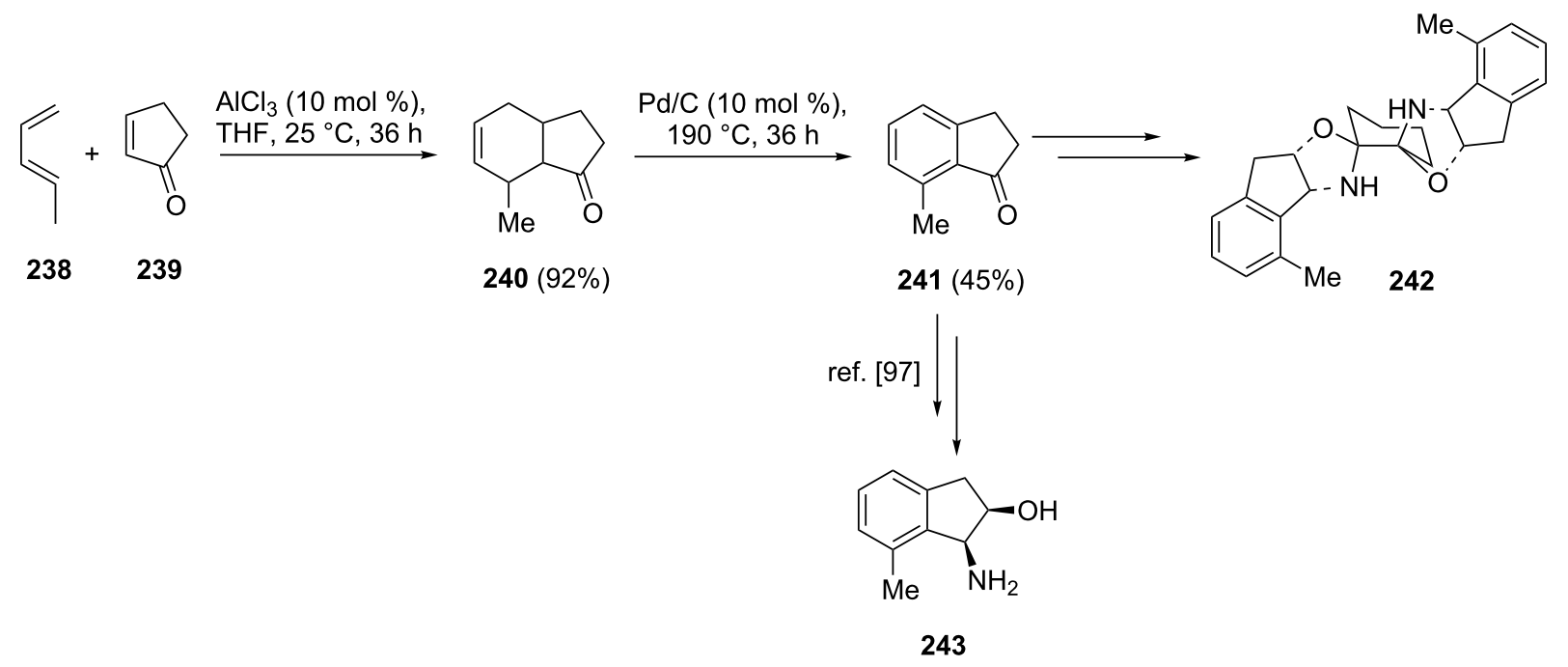

Scheme 67: Synthesis of 7-methyl substituted 1-indanone 241 from 1,3-pentadiene (238) and 2-cyclopentenone (239).

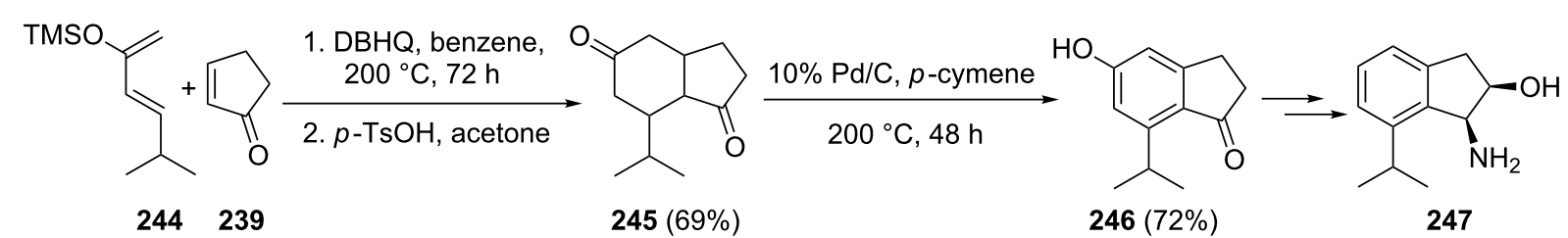

Scheme 68: Synthesis of disubstituted 1-indanone 246 from the siloxydiene 244 and 2-cyclopentenone 239. 


$$
24250
$$

Scheme 69: Synthesis of 5-hydroxy-1-indanone (250) via the Diels-Alder reaction of 1,3-diene $\mathbf{2 4 8}$ with sulfoxide 249.

Lee, Kim and Danishefsky have synthesized halogenated 1-indanones 253 from 2-halogenocyclopent-2-enones 252 and diene 251 [99]. As a result of the Diels-Alder reaction, bromoand chloro-substituted 1-indanones 253a and 253b have been obtained in $91 \%$ and $72 \%$ yield, respectively (Scheme 70 ).<smiles>C=C(C)C(=C)[C+]C1=CCCC1C(=O)OC</smiles>

251
252<smiles>[X]C12CC(C)=C(C)CC1CCCC2=O</smiles>

253 253a $X=B r(91 \%)$ 253b X $=\mathrm{Cl}(72 \%)$

Scheme 70: Synthesis of halogenated 1-indanones 253a and 253b.

Harmata et al. have synthesized 1 -indanones $\mathbf{2 5 7}$ and $\mathbf{2 5 8}$ by utilizing 2-bromocyclopentenones $\mathbf{2 5 4}$ as starting materials
[100]. First, the cyclopentadienone dimers 256a and 256b were generated from 2-bromocyclopentenones 254 using triethylamine (TEA) in trifluoroethanol (TFE). Then, the 1-indanones 257 and 258 were obtained from dimers 256 a or $256 b$ by heating in quinoline (Scheme 71).

Gacs-Baitz et al. have applied the Diels-Alder reaction between 1,2-dihydro-4-vinylnaphthalene (259) and 2-bromo-4acetoxy-2-cyclopenten-1-one (260) to synthesize 1-indanone derivative 261 (Scheme 72) [101].

Koreeda and Woski have synthesized the cyclopenta[ $\alpha]$ phenanthrene derivative $\mathbf{2 6 5}$ having the steroid framework from 1,2dihydro-7-methoxy-4-vinylnaphthalene (262) and $\alpha$-bromo substituted cyclopentenone $\mathbf{2 6 3}$ by the $\mathrm{SnCl}_{4}$-catalyzed Diels-Alder cycloaddition [102]. In this reaction, 1-indanone $\mathbf{2 6 5}$ was obtained in 59\% yield via dehydrogenation of a mixture of cycloadducts 264a-c using 10\% Pd/C (Scheme 73).

An interesting example is the Diels-Alder reaction between dihydro-3-vinylphenanthrene (266) and 4-acetoxy-2-cyclopenten-1-one (267) which led to formation of the helicene-like product 268 with the 1-indanone core (Scheme 74) [103].

The Diels-Alder reaction of $\mathbf{2 6 2}$ and phenylselenyl-substituted cyclopentenone 269 was less effective and gave 1-indanone $\mathbf{2 6 5}$ in $28 \%$ yield only (Scheme 75 ) [102]. Another example of this reaction catalyzed by a Lewis acid has also been reported [104].

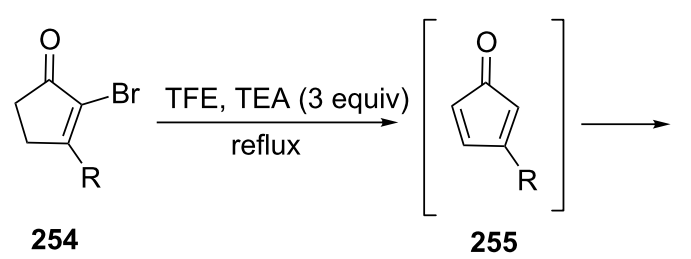<smiles>[R]C1=CC(=O)C2C(=O)C1CC2[R]</smiles>

256a<smiles>[R]C1CC(=O)C(=C)C1=C</smiles>

257<smiles>[R]c1ccc2c(c1)C([R])CC2=O</smiles><smiles>[R]C1=CC(=O)C2C(=O)C=CC1C2[R]</smiles>

256b

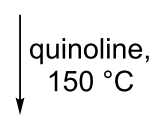<smiles>[R]c1ccc2c(c1)C(=O)CC2[R]</smiles>

$258 \mathrm{R}=\mathrm{C}_{6} \mathrm{H}_{5}(65 \%)$

257a $\mathrm{R}=\mathrm{H}(54 \%)$

257b $\mathrm{R}=\mathrm{C}_{6} \mathrm{H}_{5}(84 \%)$

257c $\mathrm{R}=3-\mathrm{MeOC}_{6} \mathrm{H}_{4}(86 \%)$

257d $\mathrm{R}=4-t-\mathrm{BuC}_{6} \mathrm{H}_{4}(84 \%)$

257e $\mathrm{R}=2,5-\mathrm{MeC}_{6} \mathrm{H}_{3}(77 \%)$

Scheme 71: Synthesis of 1-indanones 257 and 258 from 2-bromocyclopentenones 254 
<smiles>C=CC1=CCCc2ccccc21</smiles>

259<smiles>CC(=O)OC1C=C(Br)C(=O)C1</smiles>

260

\section{$\underset{\text { 1. } \mathrm{Me}\left(\mathrm{CH}_{2}\right)_{4} \mathrm{Me}, \mathrm{DCM}}{\stackrel{2 . \mathrm{Pd}, \text { triglyme }}{\longrightarrow}}$ \\ 2. Pd, triglyme}<smiles>O=C1CCc2ccc3c(ccc4ccccc43)c21</smiles>

261

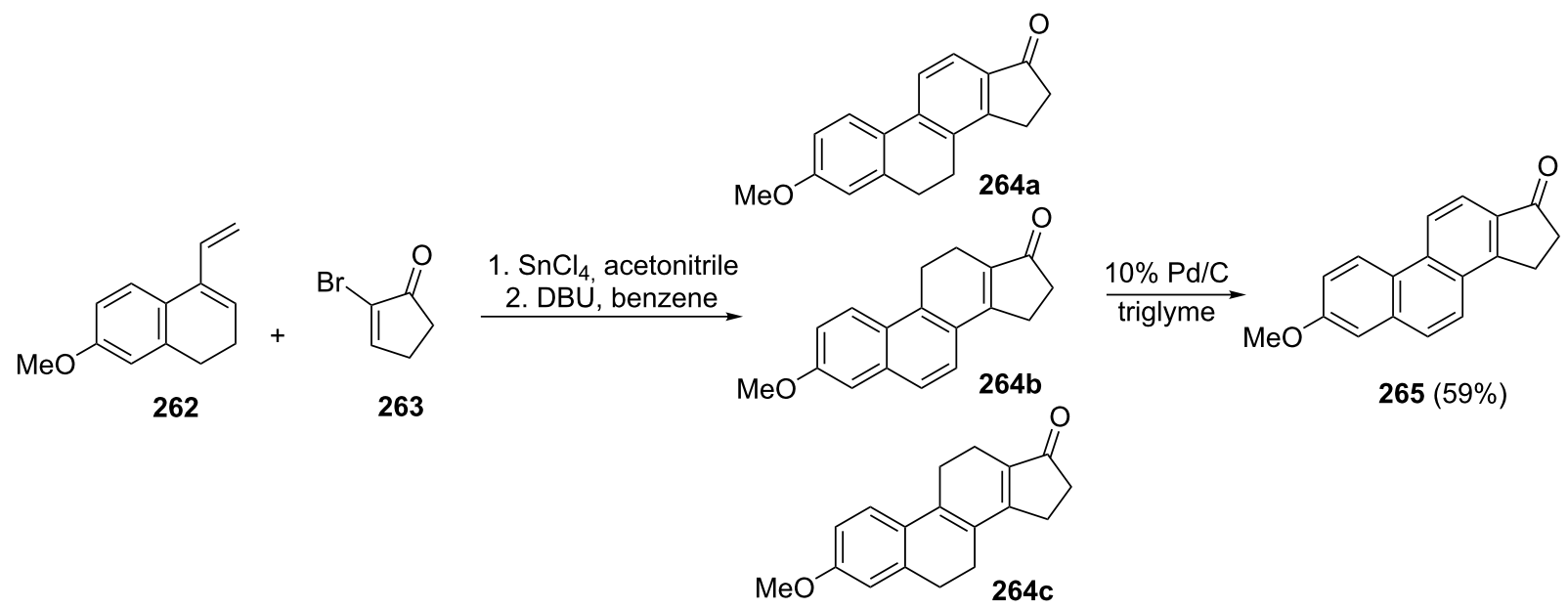

Scheme 73: Synthesis of 1-indanone 265 from 1,2-dihydro-7-methoxy-4-vinylnaphthalene (262) and bromo-substituted cyclopentenone 263.

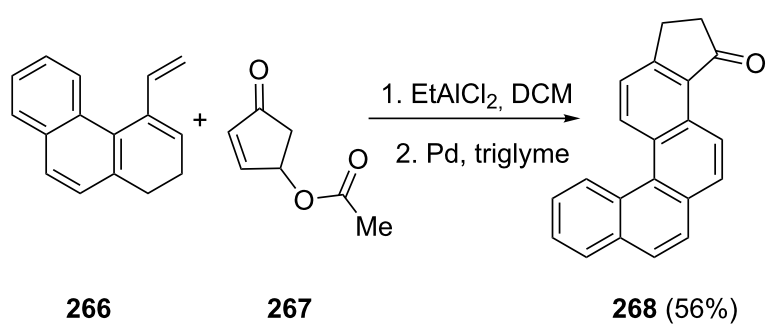

Scheme 74: Synthesis of 1-indanone 268 from dihydro-3-vinylphenanthrene 266 and 4-acetoxy-2-cyclopenten-1-one (267).
The flash vacuum pyrolysis has been applied for aromatization of $\mathbf{2 7 1}$ to afford 1-indanone $\mathbf{2 7 2}$ in 76\% yield. The former $\mathbf{2 7 1}$ was obtained from the trienone 270/270' which underwent ring closure to give the 6-membered ring [105] (Scheme 76).

\subsection{From alkynes}

$\mathrm{DBU}$ and $\mathrm{CpRu}\left(\mathrm{PPh}_{3}\right)_{2} \mathrm{Cl}$ dual catalysts enabled a one-pot annulation of aldehyde 273 and cyclopentanone (274) to give the 1-indanone derivative 276 [106]. The new catalytic reaction which replaced a previously described four-step synthesis [107], involved a tandem aldol condensation/dehydration and cyclization of the intermediate $\mathbf{2 7 5}$ to $\mathbf{2 7 6}$ (Scheme 77).

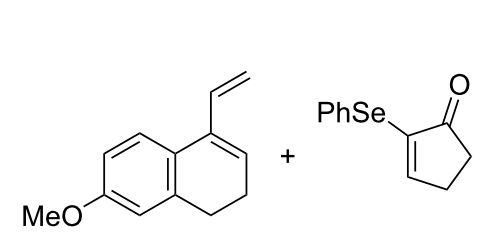

262
1. $\mathrm{SnCl}_{4}$, acetonitrile

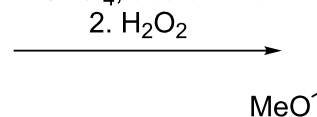

269<smiles>Cc1ccc2c(c1)CCc1c-2ccc2c1CCC2=O</smiles>

$\underset{\text { triglyme }}{\stackrel{10 \% \mathrm{Pd} / \mathrm{C}}{\longrightarrow}}$

$264 a$<smiles>COc1ccc2c(ccc3c4c(ccc32)C(=O)CC4)c1</smiles>

$265(28 \%)$

Scheme 75: Synthesis of 1-indanone $\mathbf{2 7 1}$ from phenylselenyl-substituted cyclopentenone 268. 


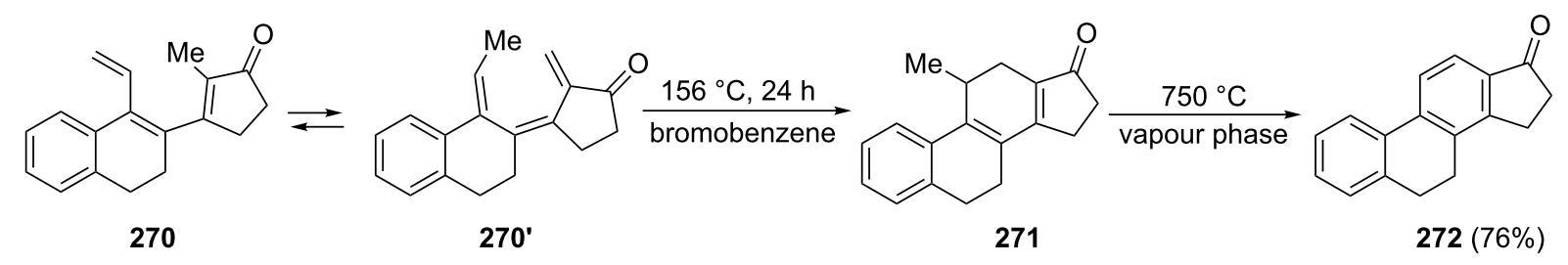

Scheme 76: Synthesis of 1-indanone $\mathbf{2 7 2}$ from the trienone $\mathbf{2 7 0}$.

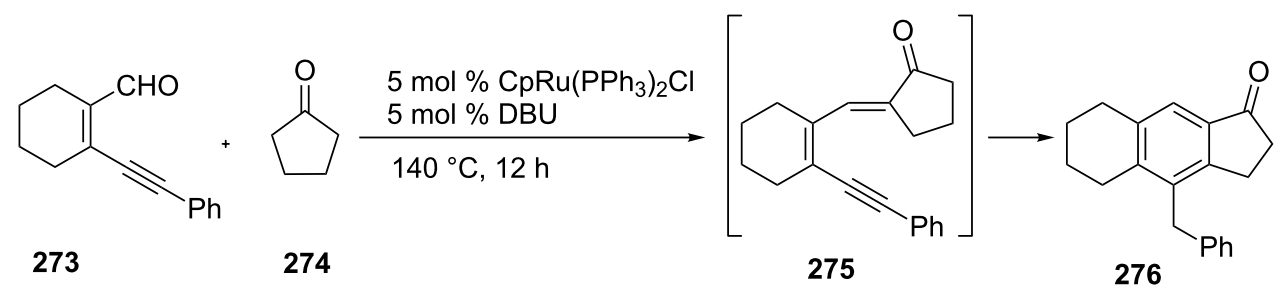

Scheme 77: Synthesis of the 1-indanone 276 from the aldehyde 273.

In 1999, Ikeda and Mori have presented a cyclotrimerization of enones (e.g., cyclopentenone 239) with alkynes in the presence of nickel and aluminum complexes [108]. This [2+2+2] cycloaddition run with a high regioselectivity and led mostly to meta isomers. The authors used, as catalytic systems, the following complexes: $\mathrm{Ni}(\mathrm{acac})_{2}, \mathrm{Ni}(\operatorname{cod})_{2}, \mathrm{Me}_{3} \mathrm{Al}$, $\mathrm{Me}_{2} \mathrm{Al}(\mathrm{OPh}), \mathrm{MeAl}(\mathrm{OPh})_{2}$ and $\mathrm{Al}(\mathrm{OPh})_{3}$. In 2000, Ikeda and Kondo have continued their studies on regioselectivity of the cyclotrimerization [109] and investigated the effects of various ligands (L) on regioselectivity and yields of this reaction (Scheme 78). In case of application of triarylphosphines $\left(\mathrm{Ph}_{3} \mathrm{P}\right.$ and $\left.\left(o-\mathrm{MeC}_{6} \mathrm{H}_{4}\right)_{3} \mathrm{P}\right)$ as ligands, only para isomers 279 were formed in moderate $33 \%$ and $49 \%$ yields, respectively. On the contrary, when oxazolines $\mathbf{2 8 0}$ or $\mathbf{2 8 1}$ were used as ligands, mainly meta isomers $\mathbf{2 7 8}$ were formed with high yields.

Cheng et al. have obtained 1-indanone $\mathbf{2 8 5}$ from octa-1,7-diyne (282) and cyclopentenone 239 as a result of Ni-complex-catalyzed $[2+2+2]$ cyclotrimerization proceeding via the intermediate 283 [110] (Scheme 79). The dimer 284 of the starting dialkyne has also been obtained.

\subsection{From o-bis(dibromomethyl)benzene}

Erenler et al. have utilized $o$-bis(dibromomethyl)benzene (286) and cyclopentenone $\mathbf{2 3 9}$ to the synthesis of benz[f]indan-1-one (287) and its bromo derivative [111]. Both compounds are

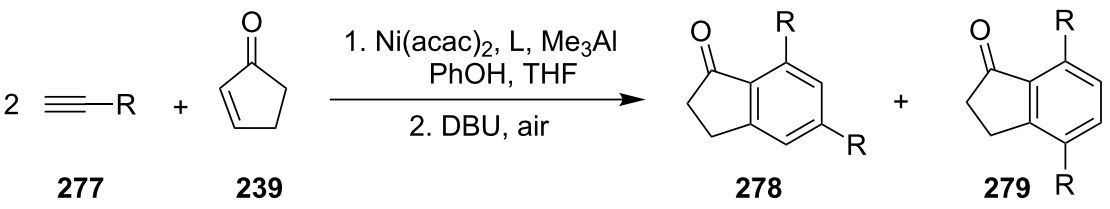

$\mathrm{R}=$ TMS-, $t$-Bu-, TBDMSOCH${ }_{2} \mathrm{CH}_{2}-$

$\mathrm{L}=\mathrm{Ph}_{3} \mathrm{P},\left(\mathrm{o}-\mathrm{MeC}_{6} \mathrm{H}_{4}\right)_{3} \mathrm{P}$,

$\mathrm{R}^{1}, \mathrm{R}^{2}=\mathrm{H}, \mathrm{Me}, \mathrm{Ph}, \mathrm{iPr}, \mathrm{Bn}$<smiles>[R]C1=NC([R])CO1</smiles>

280<smiles>O=C(c1ccccc1)C1COC(C2=NC(Cc3ccccc3)CO2)=N1</smiles>

281 


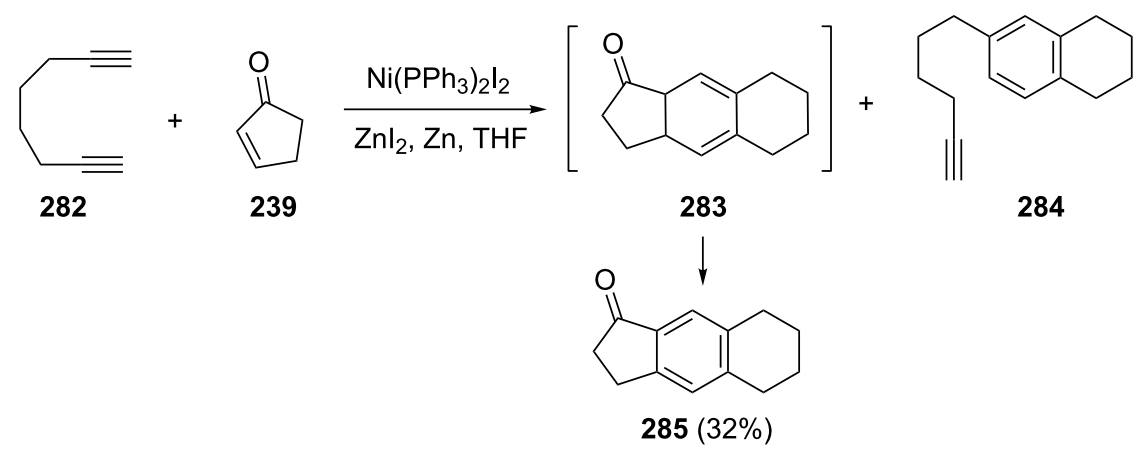

Scheme 79: Synthesis of 1-indanone 285 from octa-1,7-diyne (282) and cyclopentenone 239.

promising reagents for the synthesis of biologically active compounds (Scheme 80).<smiles>BrC(Br)c1ccccc1C(Br)Br</smiles>

286<smiles>O=C1C=CCC1[18O]</smiles>

239<smiles>O=C1CCc2cc3ccccc3cc21</smiles>

$287(60 \%)$
Scheme 80: Synthesis of benz[f]indan-1-one (287) from cyclopentenone 239 and o-bis(dibromomethyl)benzene (286).

Kubo et al. have synthesized $\mathbf{2 8 7}$ from the same substrates $\mathbf{2 3 9}$ and $\mathbf{2 8 6}$ by a slight change of reaction conditions [112].
Jones et al. have synthesized 3-methyl-substituted benz[f]indan1-one 291 in 35\% yield from $o$-bis(dibromomethyl)benzene (286) and 4-methylcyclopent-2-enone (289) (Scheme 81) [113].

\subsection{From other compounds}

Albrecht, Defoin and Siret have synthesized benz[f]indan-1-one (295) from the anthracene epidioxide 292, which underwent thermal isomerization to give the reactive intermediate $\mathbf{2 9 3}$ [114]. As a result of the Diels-Alder reaction of the latter with cyclopentenone $\mathbf{2 3 9}$, the adduct $\mathbf{2 9 4}$ was formed, which was further subjected to the TEA-induced cleavage at $100{ }^{\circ} \mathrm{C}$ to give the desired 1-indanone 295 (Scheme 82).

Masui et al. have synthesized 1-indanone $\mathbf{2 9 9}$ in 5\% as a result of the Diels-Alder reaction between homophthalic anhydride

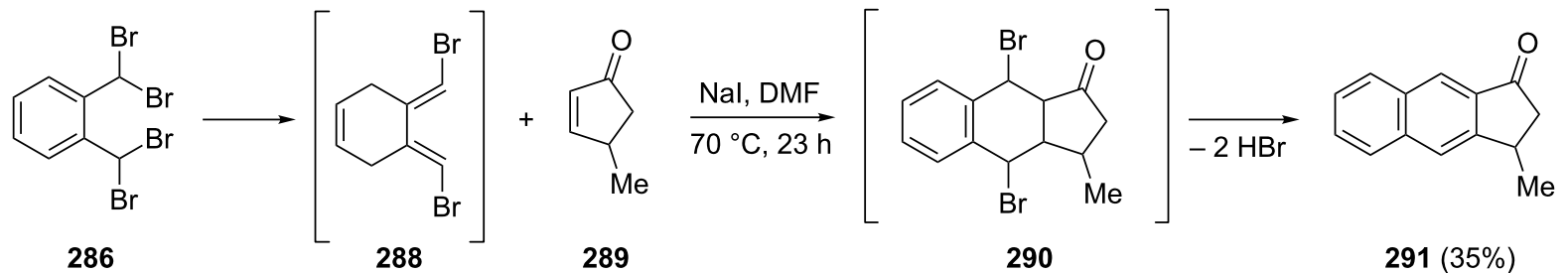

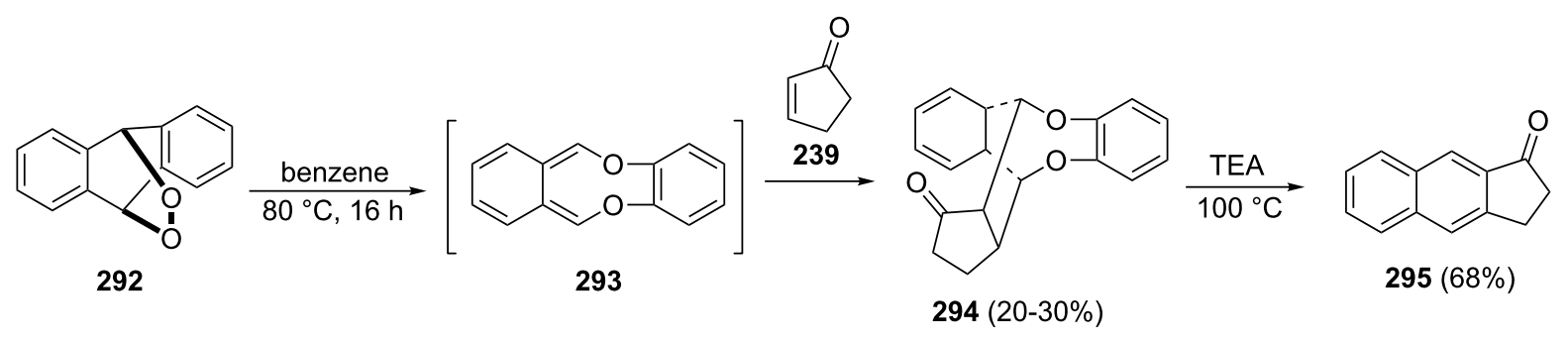


(298) and cyclopentynone 297 generated from the phosphorane 296 by the intramolecular Wittig reaction (Scheme 83) [115].

Jończyk et al. have synthesized cyano-substituted 1-indanone derivative 301 in 55\% yield under solid-liquid, phase-transfer catalysis conditions [116]. In this synthesis, 2-cyanomethylbenzaldehyde (300) was reacted with cyclopentenone 239 in the presence of powdered $\mathrm{K}_{2} \mathrm{CO}_{3}$ and Aliquat ${ }^{\circledR} 336$ as a catalyst. (Scheme 84).

\section{Construction of the 5- and 6-membered rings \\ 3.1 From alkynes}

The intramolecular, dehydro-Diels-Alder reaction of ketene dithioacetals 302 leading to formation of various benzo[f]-1indanones 303-305, has been described in 2015 by Bi et al. [117]. Modulation on the reaction parameters such as addition of DBU and the type of atmospheric gas used $\left(\mathrm{O}_{2}, \mathrm{~N}_{2}\right)$, regulated the regioselective formation of the 1-indanones 303-305 (Scheme 85).

A new, simple approach for the synthesis of natural and unnatural 1-indanones 309-316 has been proposed by Deiters et al. [118]. The key step of this synthesis was associated with [2+2 +2 ] cyclotrimerization of the dialkyne $\mathbf{3 0 6}$ with variously disubstituted alkynes $\mathbf{3 0 7}$ performed on a solid phase Tenta$\mathrm{Gel}^{\circledR}$ resin $(0.25 \mathrm{mmol} / \mathrm{g})$ in the presence of $\mathrm{Ru}$ catalyst (Scheme 86). In case of 309-316, this reaction led to the formation of mixtures of two regioisomers. The examined regioisomeric ratios $(\mathbf{a} / \mathbf{b})$ were ranged from $1: 2$ to $2: 3$ with a preference to $310 \mathrm{a}-\mathbf{3 1 5} \mathrm{a}$ regioisomers.
An interesting approach to the synthesis of 1-indanones and 1 -indenones is based on the hexadehydro-Diels-Alder (HDDA) reaction in which an alkyne reacts in the $[4+2]$ cycloaddition with diyne and forms a reactive benzyne species as a precursor of the benzene ring (Scheme 87).

This methodology has been applied in the synthesis of 1-indanones (Scheme 88 and Scheme 89).

In 2012, Hoye et al. have presented the synthesis of 1-indenone 318 via a hexadehydro-Diels-Alder (HDDA) reaction with simultaneous formation of five and six-membered rings from the tetrayne 317 (Scheme 88) [119]. In the reaction participates only three triple bonds marked by red lines. This, catalyzed by $\mathrm{MnO}_{2}$ reaction, is fully regioselective. During the cycloaddition after the formation of the five and six-membered rings, one of the tert-butyldimethylsilyl (TBS) group migrates from an oxygen to the triple bond of benzyne to give 318. In 2014, the authors have shown that the HDDA cyclization of the unsymmetrical substituted ketotetrayne 319 gives a mixture of isomeric 1-indanones $\mathbf{3 2 0}$ and 321 (Scheme 88) [120]. It is the effect of competition between two modes of the cycloaddition reaction. In the "normal" mode of this reaction, cyclization takes place between the triple bond in $\alpha, \beta$-position and the diyne in $\gamma^{\prime}, \varepsilon$ '-position to give 320. In the "abnormal" mode, the cyclization takes places between the triple bond in $\gamma$ '-position and the diyne in $\alpha, \gamma$-position to give $\mathbf{3 2 1}$.

Hoye et al. have further expanded the scope of this reaction on several other substrates which are active in the $[2+2+2]$ cycloaddition [120]. For example, the triyn 322 under hexade-

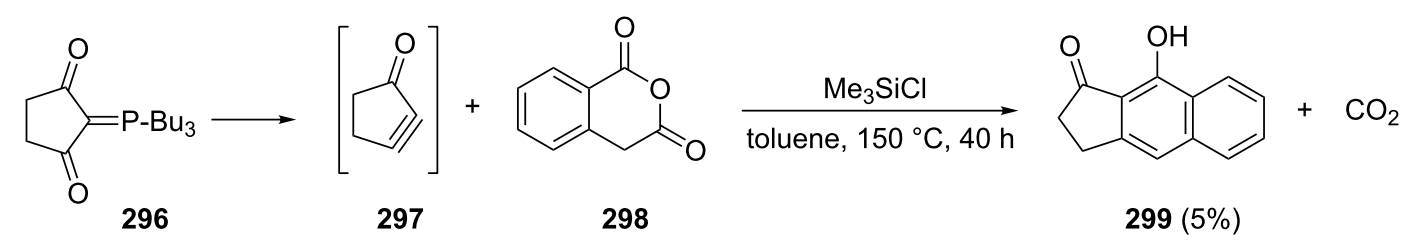

Scheme 83: Synthesis of 1-indanone 299 from homophthalic anhydride 298 and cyclopentynone 297.<smiles>N#Cc1ccccc1C=O</smiles> 
<smiles>[R]c1ccc(-c2c3c(cc4ccccc24)C(=O)C(=C2SCCS2)C3c2ccc([R])cc2)cc1</smiles>

303a-e

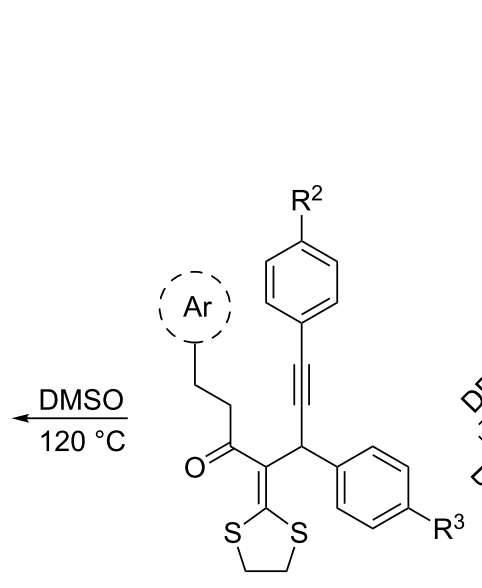

302

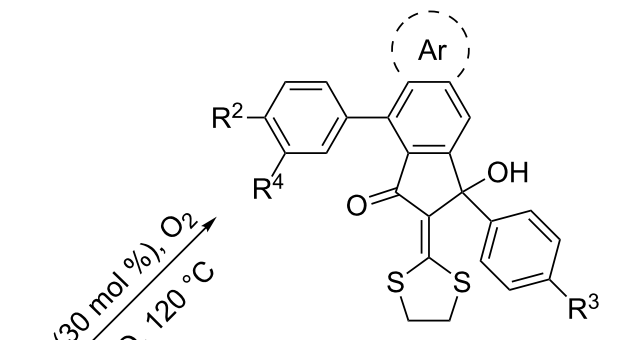

304a-e

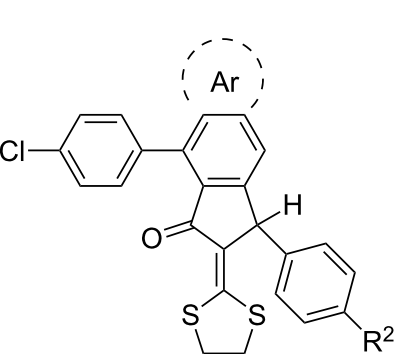

$305 a-c$

Selected examples

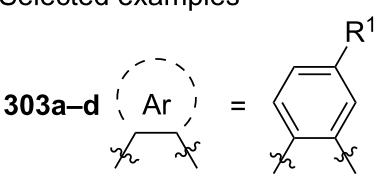

303a $R^{1}=\mathrm{Cl}, \mathrm{R}^{2}=\mathrm{H}, \mathrm{R}^{3}=\mathrm{Cl}(25 \mathrm{~h}, 86 \%)$

303b $R^{1}=R^{2}=R^{3}=H(20 h, 91 \%)$

303c $R^{1}=M e, R^{2}=R^{3}=H(16 h, 94 \%)$

303d $R^{1}=\mathrm{Cl}, \mathrm{R}^{2}=\mathrm{F}, \mathrm{R}^{3}=\mathrm{H}(22 \mathrm{~h}, 85 \%)$

$303 e$

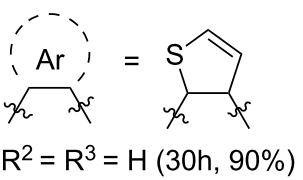<smiles>[R]c1ccc([Y])c([Y])c1</smiles>

304a $R^{1}=R^{4}=H, R^{2}=R^{3}=\mathrm{Cl}(60 \min , 86 \%)$

304b $R^{1}=R^{4}=H, R^{2}=C l, R^{3}=H(45 \min , 90 \%)$

304c $R^{1}=R^{4}=H, R^{2}=C l, R^{3}=M e ~(45 \mathrm{~min}, 88 \%)$

304d $R^{1}=M e, R^{2}=C l, R^{3}=R^{4}=H(60 \min , 85 \%)$

304e $R^{1}=F, R^{2}=C l, R^{3}=R^{4}=H(60 \min , 90 \%)$<smiles>[R]c1ccc([Y])c([Y])c1</smiles>

305a $\mathrm{R}^{1}=\mathrm{H}, \mathrm{R}^{2}=\mathrm{Cl}(30 \mathrm{~min}, 81 \%)$

305b R $\mathrm{R}^{1}=\mathrm{Me}, \mathrm{R}^{2}=\mathrm{H}(30 \mathrm{~min}, 80 \%)$

305c $R^{1}=F, R^{2}=H(30 \mathrm{~min}, 81 \%)$

Scheme 85: Synthesis of 1-indanone derivatives 303-305 from ketene dithioacetals 302.

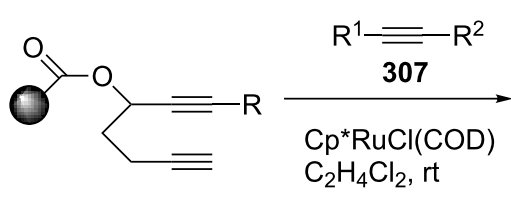

$306 \mathrm{R}=\mathrm{H}, \mathrm{R}=\mathrm{TMS}, \mathrm{R}=\mathrm{Me}$<smiles>[R]c1cc2c(cc1[R])C(OC(=O)O)CC2</smiles>

308

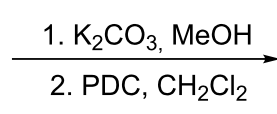<smiles>[R]c1cc2c(cc1[R])C(=O)CC2</smiles>

309-316

$309 \mathrm{R}^{1}=\mathrm{H}, \mathrm{R}^{2}=\mathrm{H}, 78 \%$

310a $R^{1}=B u, R^{2}=H ; 310 b R^{1}=H, R^{2}=B u ; 65 \%$ a/b $1: 2$

311a $R^{1}=P h, R^{2}=H ; 311 b R^{1}=H, R^{2}=P h ; 62 \%$ a/b 2:3

312a R $\mathrm{R}^{1}=\left(\mathrm{CH}_{2}\right)_{4} \mathrm{Cl}, \mathrm{R}^{2}=\mathrm{H} ; \mathbf{3 1 2} \mathbf{b} \mathrm{R}^{1}=\mathrm{H}, \mathrm{R}^{2}=\left(\mathrm{CH}_{2}\right)_{4} \mathrm{Cl} ; 72 \% \mathbf{a} / \mathbf{b} 2: 3$

313a $\mathrm{R}^{1}=\left(\mathrm{CH}_{2}\right)_{3} \mathrm{CN}, \mathrm{R}^{2}=\mathrm{H} ; 313 \mathbf{b} \mathrm{R}^{1}=\mathrm{H}, \mathrm{R}^{2}=\left(\mathrm{CH}_{2}\right)_{3} \mathrm{CN} ; 61 \%$ a/b $2: 3$

314a $R^{1}=\left(\mathrm{CH}_{2}\right) O B n, R^{2}=H ; 314 b R^{1}=H, R^{2}=\left(\mathrm{CH}_{2}\right) O B n ; 66 \%$ a/b $1: 2$

315a R ${ }^{1}=\left(\mathrm{CH}_{2}\right)$ NBoc, $\mathrm{R}^{2}=\mathrm{H} ; 3$ 315b R $\mathrm{R}^{1}=\mathrm{H}, \mathrm{R}^{2}=\left(\mathrm{CH}_{2}\right)$ NBoc; $70 \%$ a/b $1: 2$

$316 \mathrm{R}^{1}=\mathrm{CH}_{2} \mathrm{OMe}, \mathrm{R}^{2}=\mathrm{CH}_{2} \mathrm{OMe}, 58 \%$ 


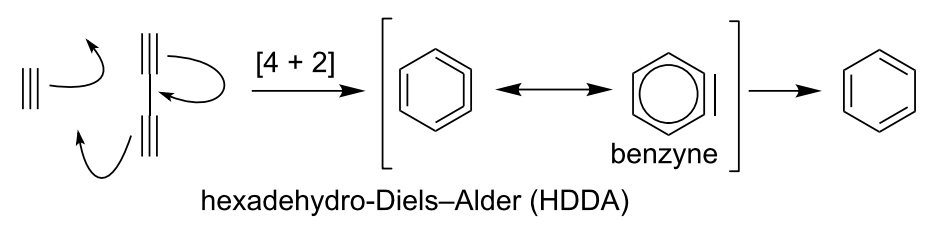

Scheme 87: Mechanism of the hexadehydro-Diels-Alder (HDDA) reaction.

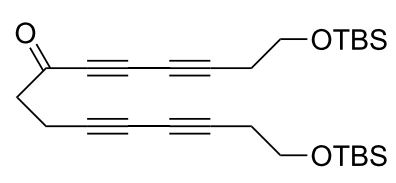

319

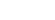

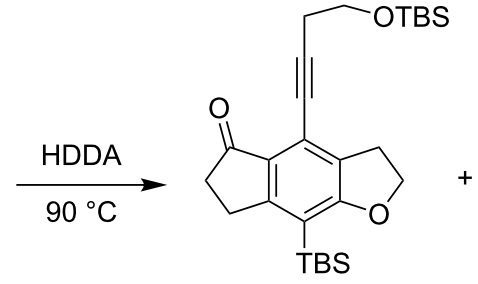

$320(62 \%)$

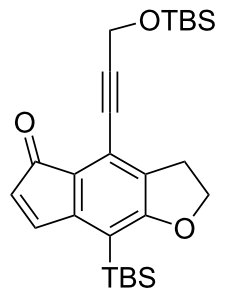

$318(53 \%)$

Scheme 88: Synthesis of 1-indenone 318 and 1-indanones 320 and 321 from tetraynes 317 and 319.

hydro-Diels-Alder (HDDA) conditions gave the corresponding 1-indanone $\mathbf{3 2 3}$ in $80 \%$ yield (Scheme 89 ).

\subsection{From furans}

Van der Eycken et al. have synthesized 1-indanone $\mathbf{3 2 8}$ by utilizing 2-methylfuran (324) as a starting compound which was converted to the Mannich adduct $\mathbf{3 2 5}$, followed by the anion exchange reaction to give ammonium hydroxide 326 [121]. The latter underwent dimerization to afford the furanocyclophane 327, which was next oxidized with meta-chloroperoxybenzoic acid ( $m$-CPBA), followed by a Diels-Alder reaction and dehydration to obtain 1 -indanone $\mathbf{3 2 8}$ in $88 \%$ yield (Scheme 90).

In 2003, Hashmi et al. have demonstrated an intramolecular gold catalyzed [4+2] cycloaddition of furans $\mathbf{3 2 9}$ with a tethered alkyne moiety [122]. The reaction was regioselective and gave 1-indanones $\mathbf{3 3 0}$ at room temperature, in good yields up to $75 \%$. The second regioisomer was formed only in 3-7\% yield (Scheme 91). 7-Hydroxy-6-methylindan-1-one $\mathbf{3 3 0}$ has later been used in the synthesis of natural sesquiterpene, jungianol isolated from Jungia malvaefolia.

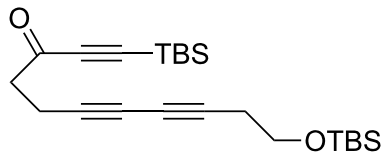

322

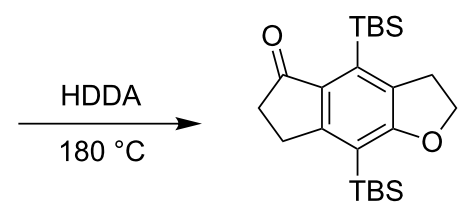

$323(80 \%)$ 


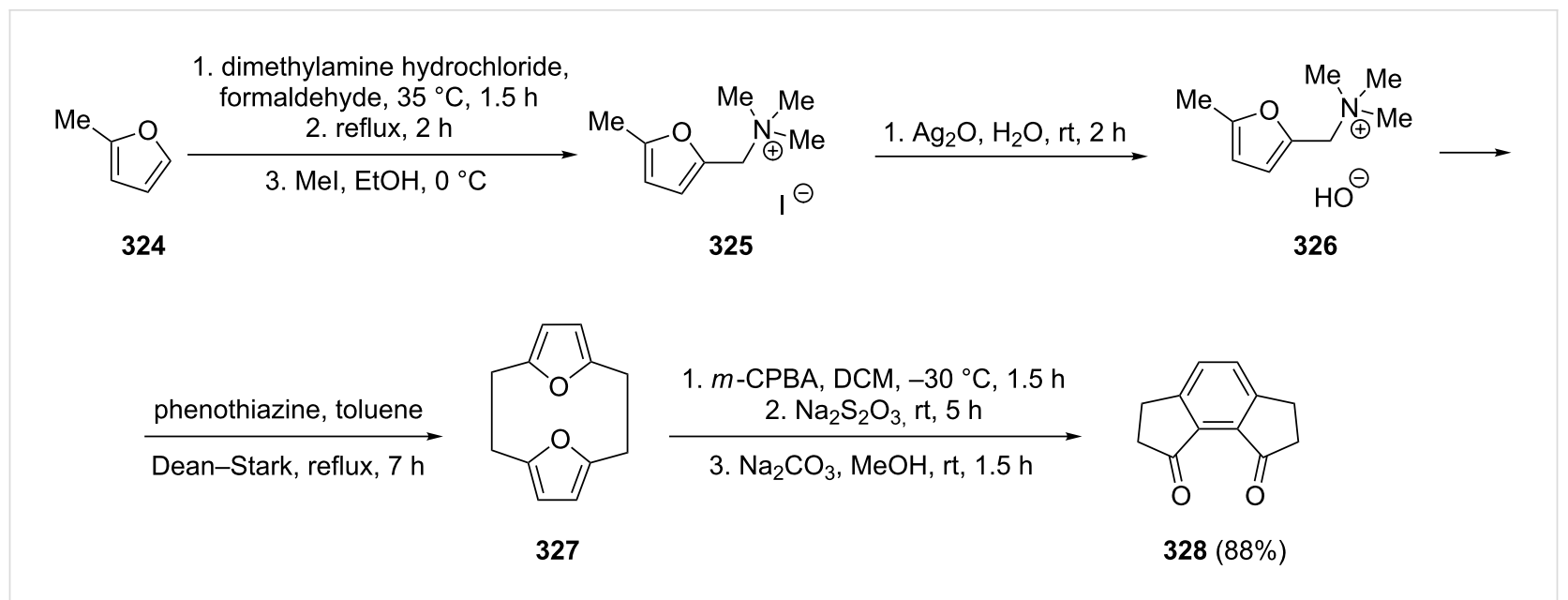

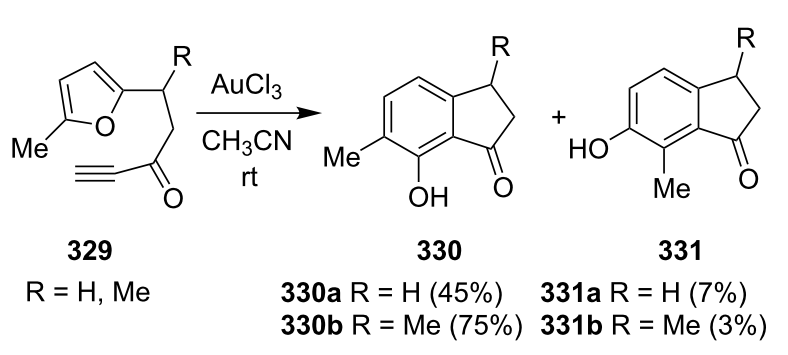

Scheme 91: Synthesis of 1-indanones $\mathbf{3 3 0}$ and $\mathbf{3 3 1}$ from furans 329

\subsection{From bicyclic compounds}

Ciabattoni, Crowley and Kende have obtained 1-indanone 333 in $52 \%$ yield by photoisomerization of benzobicyclo[3.2.2]nonatrienone 332 (Scheme 92) [123].

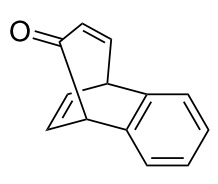

332

\section{$\underset{\text { acetonitrile, } 6 \mathrm{~h}}{\mathrm{hv} / \text { Pyrex }}$}<smiles>O=C1CCc2c1ccc1ccccc21</smiles>

$333(52 \%)$
Scheme 92: Synthesis of 1-indanone 333 from the cycloadduct $\mathbf{3 3 2}$.

\section{Functionalization of the 5- or 6-membered ring of 1 -indanones or related compounds}

Another approach to obtain bioactive 1-indanones is their functionalization. In this way, scientists have synthesized C5- and C6-alkoxy and benzyloxy-substituted 1-indanones by the alkylation reaction of 5-hydroxy-1-indanone or 6-hydroxy-1indanone with various alkyl or benzyl bromides. These 1-indanone derivatives are potential inhibitors of two separate isoforms of monoamine oxidases: MAO-A and MAO-B [124]. Monoamine oxidases (MAO) are mitochondrial enzymes that catalyze two-electron oxidation of amine substrates. MAO terminates physiological actions of amine neurotransmitters in brain; therefore, MAO inhibitors have been applied in the treatment of neurodegenerative and neuropsychiatric disorders such as Parkinson's disease and depression. The studies have shown that synthesized C6-substituted 1-indanones are effective and selective MAO-B inhibitors, while $\mathrm{C} 5$-substituted 1-indanones are less effective MAO-B inhibitors.

A 2,4-dinitrophenylhydrazone derivative of 1-indanone with a potent antimicrobial activity has been synthesized in 2014 by Obafemi et al. utilizing unsubstituted 1-indanone as a starting material [125]. This bioactive derivative has been obtained by Claisen-Schmidt reaction of 1-indanone with benzaldehyde, followed by condensation with 2,4-dinitrophenylhydrazine (DNP). This compound exhibited the best activity with the lowest MIC (minimum inhibitory concentration) values for four Gram-negative bacterial strains, such as: Pseudomonas aeruginosa, Salmonella typhimurium, Shigella flexineri and Acinetobacter calcaoceuticus anitratus $(15.6 \mu \mathrm{g} / \mathrm{mL})$, and two following Gram-positive bacterial strains: Staphylococcus aureus and Micrococcus luteus $(31.3 \mu \mathrm{g} / \mathrm{mL})$.

The reduction of 1-indenones to 1-indanones has been applied by Clark et al. [126]. The authors used bakers' yeast (Saccharomyces cerevisiae) for the reduction of 3-arylinden-1-ones 334 to obtain $(S)$-3-arylindan-1-ones $\mathbf{3 3 5}$ with high enantioselectivity (Scheme 93).

Methyl $N$-benzyl-4-methylpiperidinecarboxylate acylation with 5,6-dimethoxy-1-indanone has been applied as the key step of 
<smiles>[R]c1cc2c(cc1[R])C(c1ccc([R])c([R])c1[R])=CC2=O</smiles>

334<smiles>[R]c1cc2c(cc1[R])C(c1ccc([R8])c([R])c1[R])CC2=O</smiles>

(S)-335

$$
\begin{aligned}
& \text { 335a } R^{1}=O M e, R^{2}=H, R^{3}=H, R^{4}=O M e, R^{5}=H(81 \%) \\
& \text { 335b } R^{1}=O M e, R^{2}=H, R^{3}=H, R^{4}=M e, R^{5}=H(79 \%) \\
& \text { 335c } R^{1}=H, R^{2}=O M e, R^{3}=H, R^{4}=O M e, R^{5}=H(61 \%) \\
& \text { 335d } R^{1}=O M e, R^{2}=H, R^{3}=O M e, R^{4}=H, R^{5}=H(84 \%) \\
& \text { 335e } R^{1}=O M e, R^{2}=H, R^{3}=H, R^{4}=H, R^{5}=H(70 \%) \\
& \text { 335f } R^{1}=O M e, R^{2}=H, R^{3}=H, R^{4}=H, R^{5}=O M e(52 \%) \\
& \text { 335g } R^{1}=H, R^{2}=O M e, R^{3}=H, R^{4}=H, R^{5}=O M e(50 \%)
\end{aligned}
$$

Scheme 93: Synthesis of (S)-3-arylindan-1-ones 335.

the synthesis of 2-((1-benzyl-4-piperidinyl)hydroxymethyl)-5,6dimethoxy-1-indanone [127].

Regioselective hydrogenation of the diketone $\mathbf{3 3 6}$ followed by chemoenzymatic, dynamic kinetic resolution of the resulting rac-2-hydroxy-1-indanone (337) has been used for the synthesis of $(R)$-2-acetoxy-1-indanone (338) (Scheme 94) [128].

1-Indanones may also be synthesized by oxidation of the 1 -indane core. For example, scientists have oxidized unsubstituted indane to 1 -indanone by using a metal-free catalytic system consisting of aryl-tetrahalogenated $N$-hydroxyphthalimides (TCNHPI) and 1,4-diamino-2,3-dichloroanthraquinone (DADCAQ) in very good yield (98\%) [129]. Another catalyst, applied for the synthesis of 1-indanone, is mesoporous $\mathrm{Mn}_{0.5} \mathrm{Ce}_{0.5} \mathrm{O}_{\mathrm{x}}$ which allows a selective oxidation of hydrocarbons under mild conditions [130]. This compound catalyzed the oxidation of unsubstituted indane in which 1-indanone was obtained as the main product along with 1-indanol. An efficient synthesis using microreactors for oxidation of benzylic compounds such as xanthene, fluorene, 3,4-dihydro- $2 H$-naphtha- lene, indane and diphenylmethane has been proposed by Jia et al. [131]. Thus, indane was oxidized to 1-indanone in high yield (94\%) by oxygen formed in the reaction of $\mathrm{NaClO}$ with tertbutyl hydroperoxide.

2,3-Dichloro-5,6-dicyano-1,4-benzoquinone (DDQ) has also been used to oxidize the indane core [132]. This reaction constituted the first step in the synthesis of hydroxy-substituted, carcinogenic cyclopenta $[\alpha]$ phenanthrene.

Harvey and Lee have reported a synthesis of carcinogenic cyclopenta $[\alpha]$ phenanthrenes containing a 1-indanone core 339 [133] (Figure 6).

Other catalysts applied for the oxidation of indanes or 1-indanols to 1-indanones are ruthenium catalysts immobilized on a solid phase. Thus, a poly(ethylene glycol)-supported ruthenium complex catalyzed oxidation of indane to 1-indanone in 99\% yields [134]. $\mathrm{SiO}_{2}$-supported iodoarene- $\mathrm{RuCl}_{3}$ bifunctional catalyst catalyzed the conversion of indane into 1 -indanone in $92 \%$ yield [135].<smiles>O=C1Cc2ccccc2C1=O</smiles>
336

\section{$\underset{\text { conversion } 80 \%}{\stackrel{\mathrm{H}_{2}\left(1 \mathrm{~atm} \text { ) }, \mathrm{Pd} / \mathrm{Al}_{2} \mathrm{O}_{3}\right.}{\longrightarrow}}$}<smiles>O=C1c2ccccc2CC1O</smiles>

rac-337 selectivity $95 \%$

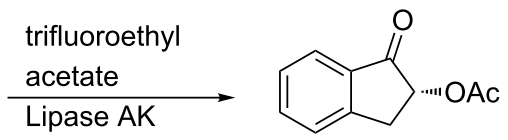

(R)-338 (33-52\%) enantiopurity: 86-92\% 
<smiles>[R]c1ccc2c(ccc3c4c(cc([R])c32)CCC4=O)c1</smiles>

339

339a $R^{1}=R^{2}=H(90 \%)$

339b $R^{1}=M e ; R^{2}=H(86 \%)$

339c $R^{1}=H ; R^{2}=M e O(90 \%)$

Figure 6: Chemical structures of obtained cyclopenta[a]phenanthrenes 339 .

1-Indanol tricarbonylchromiums have been oxidized with $\mathrm{MnO}_{2}$ to optically pure 1-indanone tricarbonylchromium derivatives by Jaouen and Meyer [136].

Voskoboynikov et al. have also applied the oxidation of the indane core to obtain benzoindanone 343 [137]. As a result of the $\mathrm{TiCl}_{4}$-catalyzed reaction of arylacetaldehyde $\mathbf{3 4 0}$ with 1-trimethylsilyloxycyclopentene (341), cyclopenta[ $\alpha$ ]naphthalene $\mathbf{3 4 2}$ was formed (Scheme 95) [137]. The latter was then oxidized to the benzoindanone 341 with dichlorodicyanobenzoquinone (DDQ).

\section{Conclusion}

This article is the first comprehensive work reviewing original and patent literature of synthetic methods and biological applications of 1-indanones. It has been shown that these bioactive molecules may be obtained from a variety of starting materials. The commonly used reactions leading to the formation of the title compounds are Nazarov, Knoevenagel, Diels-Alder, and Friedel-Crafts alkylation and acylation reactions. The structural diversity of 1-indanones implies various biological responses and these compounds may be applied in agriculture and medicine. Some of the 1-indanone derivatives may constitute a new hope, as future drugs, for the patients suffering from Alzheimer's and Parkinson's diseases, and those infected with hepatitis C virus. Single applications for organic optoelectronics have also been reported. Due to the wide application potential, 1-indanones are interesting objects for further investigations and it is desirable to design new methods for their synthesis.

\section{References}

1. Nagle, D. G.; Zhou, Y.-D.; Park, P. U.; Paul, V. J.; Rajbhandari, I.; Duncan, C. J. G.; Pasco, D. S. J. Nat. Prod. 2000, 63, 1431-1433. doi:10.1021/np000216e

2. Cossy, J.; Bellotti, D.; Maguer, A. Synlett 2003, 1515-1517. doi:10.1055/s-2003-40868

3. Yu, H.; Kim, I. J.; Folk, J. E.; Tian, X.; Rothman, R. B.; Baumann, M. H.; Dersch, C. M.; Flippen-Anderson, J. L.; Parrish, D.; Jacobson, A. E.; Rice, K. C. J. Med. Chem. 2004, 47, 2624-2634. doi:10.1021/jm0305873

4. Ahmed, N. Synthetic Advances in the Indane Natural Product Scaffolds as Drug Candidates: A Review. In Studies in Natural Products Chemistry; Atta-Ur-Rahman, Ed.; Elsevier, 2016; Vol. 51, pp 1-535.

5. Finkielsztein, L. A.; Castro, E. F.; Fabian, L. E.; Moltrasio, G. Y.; Campos, R. H.; Cavallaro, L. V.; Moglioni, A. G. Eur. J. Med. Chem. 2008, 43, 1767-1773. doi:10.1016/j.ejmech.2007.10.023

6. Fillion, E.; Fishlock, D.; Wilsily, A.; Goll, J. M. J. Org. Chem. 2005, 70, 1316-1327. doi:10.1021/jo0483724

7. Petrignet, J.; Roisnel, T.; Gree, R. Chem. - Eur. J. 2007, 13, 7374-7384. doi:10.1002/chem.200700613

8. Huang, L.; Miao, H.; Sun, Y.; Meng, F.; Li, X. Eur. J. Med. Chem. 2014, 87, 429-439. doi:10.1016/j.ejmech.2014.09.081

9. Chan, L.; Das, S. K.; Reddy, J.; Poisson, C.; Proulx, M.; Pereira, O.; Courchesne, M.; Roy, C.; Wang, W.; Siddiqui, A.; Yannopoluos, C. G.; Nguyen-Ba, N.; Labrecque, D.; Betchell, R.; Hamel, M.; Courtemanche-Asselin, P.; L'Heureux, L.; David, M.; Nicolas, O.; Brunette, S.; Bilimoria, D.; Bédard, J. Bioorg. Med. Chem. Lett. 2004, 14, 793-796. doi:10.1016/j.bmcl.2003.10.067

10. Chan, L.; Pereira, O.; Reddy, T. J.; Das, S. K.; Poisson, C.; Courchesne, M.; Proulx, M.; Siddiqui, A.; Yannopoulos, C. G.; Nguyen-Ba, N.; Roy, C.; Nasturica, D.; Moinet, C.; Bethell, R.; Hamel, M.; L'Heureux, L.; David, M.; Nicolas, O.;

Courtemanche-Asselin, P.; Brunette, S.; Bilimoria, D.; Bédard, J. Bioorg. Med. Chem. Lett. 2004, 14, 797-800. doi:10.1016/j.bmcl.2003.10.068

11. Konek, F.; Janovics, M. Mat. Termeszettud. Ert. 1926, 42, 210-223.

12. Price, C. C.; Lewis, F. M. J. Am. Chem. Soc. 1939, 61, 2553-2554. doi:10.1021/ja01878a506

13. Parham, W. E.; Jones, L. D.; Sayed, Y. J. Org. Chem. 1975, 40, 2394-2399. doi:10.1021/jo00904a029

14. Cui, D.-M.; Zhang, C.; Kawamura, M.; Shimada, S. Tetrahedron Lett. 2004, 45, 1741-1745. doi:10.1016/j.tetlet.2003.12.085

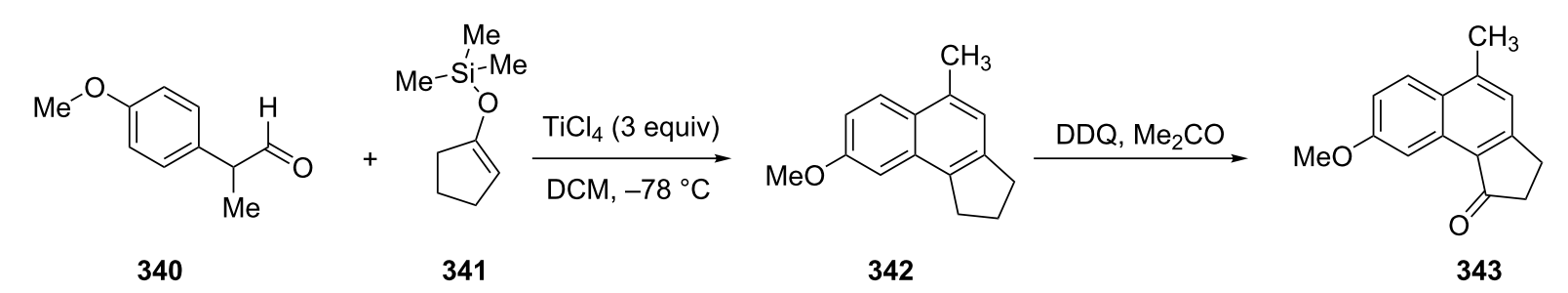

Scheme 95: Synthesis of the benzoindanone $\mathbf{3 4 3}$ from arylacetaldehyde $\mathbf{3 4 0}$ with 1-trimethylsilyloxycyclopentene (341) 
15. Etomi, N.; Kumamoto, T.; Nakanishi, W.; Ishikawa, T. Beilstein J. Org. Chem. 2008, 4, No. 15. doi:10.3762/bjoc.4.15

16. Carter, R. H.; Colyer, R. M.; Hill, R. A.; Staunton, J. J. Chem. Soc., Perkin Trans. 1 1976, 1438-1441. doi:10.1039/P19760001438

17. Prakash, G. K. S.; Paknia, F.; Vaghoo, H.; Rasul, G.; Mathew, T.; Olah, G. A. J. Org. Chem. 2010, 75, 2219-2226. doi:10.1021/jo9026275

18. Huang, Y.-S.; Liu, J.-Q.; Zhang, L.-J.; Lu, H.-L. Ind. Eng. Chem. Res. 2012, 51, 1105-1109. doi:10.1021/ie202369w

19. Neudeck, H. K. Monatsh. Chem. 1996, 127, 185-200. doi:10.1007/BF00807400

20. da Silva Barbosa, J.; da Silva, G. V. J.; Constantino, M. G. Tetrahedron Lett. 2015, 56, 4649-4652. doi:10.1016/j.tetlet.2015.06.061

21. Xu, W.; Wang, Y.; Zhan, C.; Wang, H.; Yang, X. Synthetic method of 5-chloro-1-indanone. Chinese Patent CN104,910,001 A, Sept 16, 2015.

22. Tran, P. H.; Huynh, V. H.; Hansen, P. E.; Chau, D.-K. N.; Le, T. N. Asian J. Org. Chem. 2015, 4, 482-486.

23. Yoo, K.; Kim, H.; Yun, J. Chem. - Eur. J. 2009, 15, 11134-11138. doi:10.1002/chem.200901262

24. Amagat, M. P. Bull. Soc. Chim. Fr. 1927, 41, 940-943.

25. Mayer, F.; Müller, P. Ber. Dtsch. Chem. Ges. B 1927, 60, 2278-2283. doi:10.1002/cber.19270601008

26. Narine, A. A.; Wilson, P. D. Can. J. Chem. 2005, 83, 413-419. doi:10.1139/v05-052

27. Yamato, T.; Hideshima, C.; Prakash, G. K. S.; Olah, G. A. J. Org. Chem. 1991, 56, 3955-3957. doi:10.1021/jo00012a033

28. Wu, C.; Nakamura, H.; Murai, A.; Inouye, S. Tetrahedron 2001, 57 , 9575-9583. doi:10.1016/S0040-4020(01)00980-2

29. Chen, F.; Li, S. Preparation method of 5-hydroxy-1-indanone. Chinese Patent CN105,237,381 A, Jan 13, 2016.

30. Roberts, L. R.; Bryans, J.; Conlon, K.; McMurray, G.; Stobie, A.; Whitlock, G. A. Bioorg. Med. Chem. Lett. 2008, 18, 6437-6440. doi:10.1016/j.bmcl.2008.10.066

31. Kabdulov, M. A.; Amsharov, K. Y.; Jansen, M. Tetrahedron 2010, 66, 8587-8593. doi:10.1016/j.tet.2010.09.055

32. Gilmore, R. C., Jr. J. Am. Chem. Soc. 1951, 73, 5879-5880. doi:10.1021/ja01156a521

33. Negishi, E.; Zhang, Y.; Shimoyama, I.; Wu, G. J. Am. Chem. Soc. 1989, 111, 8018-8020. doi:10.1021/ja00202a055

34. Rinehart, K. L., Jr.; Gustafson, D. H. J. Org. Chem. 1960, 25, 1836. doi:10.1021/jo01080a621

35. Zabadal, M.; Pelliccioli, A. P.; Klán, P.; Wirz, J. J. Phys. Chem. A 2001, 105, 10329-10333. doi:10.1021/jp010220e

36. Nakamura, S.; Sugimoto, H.; Ohwada, T. J. Org. Chem. 2008, 73, 4219-4224. doi:10.1021/jo800674h

37. Zhou, D.; Matsuya, Y. Huaxue Tongbao 2013, 76, 286-288.

38. Martinez, A.; Fernández, M.; Estévez, J. C.; Estévez, R. J.; Castedo, L. Tetrahedron 2005, 61, 1353-1362. doi:10.1016/j.tet.2004.10.044

39. Itoh, T.; Mase, T.; Nishikata, T.; Iyama, T.; Tachikawa, H.; Kobayashi, Y.; Yamamoto, Y.; Miyaura, N. Tetrahedron 2006, 62, 9610-9621. doi:10.1016/j.tet.2006.07.075

40. Nassar-Hardy, L.; Fabre, S.; Amer, A. M.; Fouquet, E.; Felpin, F.-X. Tetrahedron Lett. 2012, 53, 338-341. doi:10.1016/j.tetlet.2011.11.042

41. Saravanan, V. S.; Selvan, P. S.; Gopal, N.; Gupta, J. K. Asian J. Chem. 2006, 18, 2597-2604.
42. Hammen, P. D.; Milne, G. M., Jr. 2-Aminomethyleneindanone analgesic agents. U.S. Patent US4,064,272 A, Dec 20, 1977.

43. Albrecht, R.; Kessler, H.; Schroder, E. Nitrofurylpyrimidines. U.S. Patent US3,846,428 A, Nov 5, 1974.

44. Saxena, H. O.; Faridi, U.; Srivastava, S.; Kumar, J. K.; Darokar, M. P.; Luqman, S.; Chanotiya, C. S.; Krishna, V.; Negi, A. S.; Khanuja, S. P. S. Bioorg. Med. Chem. Lett. 2008, 18, 3914-3918. doi:10.1016/j.bmcl.2008.06.039

45. Charris, J. E.; Lobo, G. M.; Camacho, J.; Ferrer, R.; Barazarte, A.; Dominguez, J. N.; Gamboa, N.; Rodrigues, J. R.; Angel, J. E. Lett. Drug Des. Discovery 2007, 4, 49-54. doi:10.2174/157018007778992865

46. Patel, A.; Giles, D.; Basavarajaswamy, G.; Sreedhar, C.; Patel, A Med. Chem. Res. 2012, 21, 4403-4411. doi:10.1007/s00044-012-9973-5

47. Sharma, A. K.; Subramani, A. V.; Gorman, C. B. Tetrahedron 2007, 63, 389-395. doi:10.1016/j.tet.2006.10.065

48. Lou, T.; Liao, E.-T.; Wilsily, A.; Fillion, E. Org. Synth. 2012, 89, 115-125. doi:10.15227/orgsyn.089.0115

49. Cheng, Y.; Peng, J.-H.; Li, Y.-J.; Shi, X.-Y.; Tang, M.-S.; Tan, T.-Y. J. Org. Chem. 2011, 76, 1844-1851. doi:10.1021/jo102582a

50. Sánchez-Larios, E.; Holmes, J. M.; Daschner, C. L.; Gravel, M. Org. Lett. 2010, 12, 5772-5775. doi:10.1021/ol102685u

51. Sánchez-Larios, E.; Holmes, J. M.; Daschner, C. L.; Gravel, M. Synthesis 2011, 1896-1904. doi:10.1055/s-0030-1260031

52. González, M. L.; Sánchez-Vergara, M. E.; Álvarez-Bada, J. R.; Chávez-Uribe, M. I.; Toscano, R. A.; Álvarez-Toledano, C. J. Mater. Chem. C 2014, 2, 5607-5614. doi:10.1039/c4tc00599f

53. Hoshimoto, Y.; Hayashi, Y.; Suzuki, H.; Ohashi, M.; Ogoshi, S. Angew. Chem., Int. Ed. 2012, 51, 10812-10815. doi:10.1002/anie.201206186

54. Gevorgyan, V.; Quan, L. G.; Yamamoto, Y. Tetrahedron Lett. 1999, 40, 4089-4092. doi:10.1016/S0040-4039(99)00656-5

55. Goudreau, N.; Cameron, D. R.; Déziel, R.; Haché, B.; Jakalian, A.; Malenfant, E.; Naud, J.; Ogilvie, W. W.; O'Meara, J.; White, P. W.; Yoakim, C. Bioorg. Med. Chem. 2007, 15, 2690-2700. doi:10.1016/j.bmc.2007.01.036

56. Sun, F.-G.; Ye, S. Synlett 2011, 1005-1009. doi:10.1055/s-0030-1259707

57. Wessig, P.; Glombitza, C.; Müller, G.; Teubner, J. J. Org. Chem. 2004, 69, 7582-7591. doi:10.1021/jo040173x

58. Yu, Y.-N.; Xu, M.-H. J. Org. Chem. 2013, 78, 2736-2741. doi:10.1021/jo302656s

59. Wintgens, V.; Netto-Ferreira, J. C.; Scaiano, J. C. Photochem. Photobiol. Sci. 2002, 1, 184-189. doi:10.1039/b108116k

60. Wagner, P. J.; Park, B.-S.; Sobczak, M.; Frey, J.; Rappoport, Z. J. Am. Chem. Soc. 1995, 117, 7619-7629. doi:10.1021/ja00134a006

61. Brands, M.; Bruckmann, J.; Krüger, C.; Butenschön, H. J. Chem. Soc., Chem. Commun. 1994, 999-1000. doi:10.1039/C39940000999

62. Kaupp, G. Angew. Chem. 1992, 104, 435-437. doi:10.1002/ange.19921040408

63. Gao, S.; Wang, Q.; Chen, C. J. Am. Chem. Soc. 2009, 131, 1410-1412. doi:10.1021/ja808110d

64. Bhattacharya, A.; Segmuller, B.; Ybarra, A. Synth. Commun. 1996, 26, 1775-1784. doi:10.1080/00397919608002617

65. Nie, J.; Zhu, H.-W.; Cui, H.-F.; Hua, M.-Q.; Ma, J.-A. Org. Lett. 2007, 9, 3053-3056. doi:10.1021/ol071114j 
66. Prakasham, A. P.; Saxena, A. K.; Lugman, S.; Chanda, D.; Kaur, T.; Gupta, A.; Yadav, D. K.; Chanotiya, C. S.; Shanker, K.; Khan, F.; Negi, A. S. Bioorg. Med. Chem. 2012, 20, 3049-3057. doi:10.1016/j.bmc.2012.02.057

67. Seery, M. K.; Draper, S. M.; Kelly, J. M.; McCabe, T.; McMurry, T. B. H. Synthesis 2005, 470-474. doi:10.1055/s-2005-861799

68. Lawrence, N. J.; Armitage, E. S. M.; Greedy, B.; Cook, D.; Ducki, S.; McGown, A. T. Tetrahedron Lett. 2006, 47, 1637-1640. doi:10.1016/j.tetlet.2005.12.110

69. He, W.; Herrick, I. R.; Atesin, T. A.; Caruana, P. A.; Kellenberger, C. A.; Frontier, A. J. J. Am. Chem. Soc. 2008, 130, 1003-1011. doi:10.1021/ja077162g

70. Giese, S.; West, F. G. Tetrahedron Lett. 1998, 39, 8393-8996. doi:10.1016/S0040-4039(98)01934-0

71. Giese, S.; West, F. G. Tetrahedron 2000, 56, 10221-10228. doi:10.1016/S0040-4020(00)00866-8

72. Vaidya, T.; Atesin, A. C.; Herrick, I. R.; Frontier, A. J.; Eisenberg, R. Angew. Chem., Int. Ed. 2010, 122, 3435-3438. doi:10.1002/ange.201000100

73. Szczęsna, D.; Koprowski, M.; Różycka-Sokołowska, E.; Marciniak, B.; Bałczewski, P. Synlett 2017, 28, 113-116. doi:10.1055/s-0036-1588599

74. Smith, A. B.; Agosta, W. C. J. Am. Chem. Soc. 1973, 95, 1961-1968. doi:10.1021/ja00787a041

75. Zhu, Y.-P.; Cai, Q.; Jia, F.-C.; Liu, M.-C.; Gao, Q.-H.; Meng, X.-G.; Wu, A.-X. Tetrahedron 2014, 70, 9536-9544. doi:10.1016/j.tet.2014.10.052

76. Shintani, R.; Okamoto, K.; Hayashi, T. J. Am. Chem. Soc. 2005, 127, 2872-2873. doi:10.1021/ja042582g

77. Shintani, R.; Yashio, K.; Nakamura, T.; Okomoto, K.; Shimada, T.; Hayashi, T. J. Am. Chem. Soc. 2006, 128, 2772-2773. doi:10.1021/ja056584s

78. Mitchell, D.; Liebeskind, L. S. J. Am. Chem. Soc. 1990, 112, 291-296. doi:10.1021/ja00157a045

79. Yanagimachi, K. S.; Stafford, D. E.; Dexter, A. F.; Sinskey, A. J.; Drew, S.; Stephanopoulos, G. Eur. J. Biochem. 2001, 268, 4950-4960. doi:10.1046/j.0014-2956.2001.02426.x

80. Baur, F.; Beattie, D.; Beer, D.; Bentley, D.; Bradley, M.; Bruce, I.; Charlton, S. J.; Cuenoud, B.; Ernst, R.; Fairhurst, R. A.; Faller, B.; Farr, D.; Kaller, T.; Fozard, J. R.; Fullerton, J.; Garman, S.; Hatto, J.; Hayden, C.; He, H.; Howes, C.; Janus, D.; Jiang, Z.; Lewis, C.; Loeuillet-Ritzler, F.; Moser, H.; Reilly, J.; Steward, A.; Sykes, D.; Tedaldi, L.; Trifilieff, A.; Tweed, M.; Watson, S.; Wissler, E.; Wyss, D. J. Med. Chem. 2010, 53, 3675-3684. doi:10.1021/jm100068m

81. Hsu, S.-C.; Narsingam, M.; Lin, Y.-F.; Hsu, F.-L.; Uang, B.-J. Tetrahedron 2013, 69, 2572-2576. doi:10.1016/j.tet.2013.01.055

82. Saito, A.; Umakoshi, M.; Yagyu, N.; Hanzawa, Y. Org. Lett. 2008, 10, 1783-1785. doi:10.1021/ol800539a

83. Xi, C.; Liu, Y. Preparation of 3-aryl-1-indanone derivative. Chinese Patent CN105,348,062 A, Feb 2, 2016.

84. Pletnev, A. A.; Larock, R. C. Tetrahedron Lett. 2002, 43, 2133-2136. doi:10.1016/S0040-4039(02)00247-2

85. Kim, K.-H.; Kim, S.-H.; Lee, K.-Y.; Kim, J.-N. Bull. Korean Chem. Soc. 2011, 32, 1387-1390. doi:10.5012/bkcs.2011.32.4.1387

86. Takahashi, T.; Tsutsui, H.; Tamura, M.; Kitagaki, S.; Nakajima, M.; Hashimoto, S. Chem. Commun. 2001, 1604-1605. doi:10.1039/b103747c

87. Bonnaud, B.; Funes, P.; Jubault, N.; Vacher, B. Eur. J. Org. Chem. 2005, 3360-3369. doi:10.1002/ejoc.200500143
88. Watanabe, N.; Ikeno, A.; Minato, H.; Nakagawa, H.; Kohayakawa, C.; Tsuji, J.-i. J. Med. Chem. 2003, 46, 3961-3964. doi:10.1021/jm034134+

89. Natori, Y.; Anada, M.; Nakamura, S.; Nambu, H.; Hashimoto, S. Heterocycles 2006, 70, 635-646. doi:10.3987/COM-06-S(W)58

90. Ahmed, N.; Babu, B. V.; Kumar, H. Synthesis 2011, 2471-2477. doi:10.1055/s-0030-1260091

91. Ahmed, N.; Pathe, G. K.; Babu, B. V. Tetrahedron Lett. 2014, 55, 3683-3687. doi:10.1016/j.tetlet.2014.05.009

92. Shao, Y.; Yang, C.; Gui, W.; Liu, Y.; Xia, W. Chem. Commun. 2012, 48, 3560-3562. doi:10.1039/c2cc17960a

93. Krawczyk, E.; Mielniczak, G.; Owsianik, K.; Łuczak, J. Tetrahedron: Asymmetry 2012, 23, 1480-1489. doi:10.1016/j.tetasy.2012.09.012

94. Rosa, D.; Orellana, A. Chem. Commun. 2012, 48, 1922-1924. doi:10.1039/c2cc16758a

95. Sun, R.; Jiang, Y.; Tang, X.-Y.; Shi, M. Chem. - Eur. J. 2016, 22, 5727-5733. doi:10.1002/chem.201504914

96. Xu, H.; Wolf, C. Angew. Chem., Int. Ed. 2011, 50, 12249-12252. doi:10.1002/anie.201105778

97. Kobayashi, T.; Tanaka, K.; Miwa, J.; Katsumura, S. Tetrahedron: Asymmetry 2004, 15, 185-188. doi:10.1016/j.tetasy.2003.10.029

98. Danishefsky, S.; Harayama, T.; Singh, R. K. J. Am. Chem. Soc. 1979, 101, 7008-7012. doi:10.1021/ja00517a038

99. Lee, J. H.; Kim, W. H.; Danishefsky, S. J. Tetrahedron Lett. 2010, 51, 4653-4654. doi:10.1016/j.tetlet.2010.06.135

100. Harmata, M.; Barnes, C. L.; Brackley, J.; Bohnert, G.; Kirchhoefer, P.; Kürti, L.; Rashatasakhon, P. J. Org. Chem. 2001, 66, 5232-5236. doi:10.1021/jo015671+

101. Gacs-Baitz, E.; Minuti, L.; Taticchi, A. Tetrahedron 1994, 50, 10359-10366. doi:10.1016/S0040-4020(01)81768-3

102. Woski, S. A.; Koreeda, M. J. Org. Chem. 1992, 57, 5736-5741. doi:10.1021/jo00047a030

103. Gacs-baitz, E.; Minuti, L.; Taticchi, A. Polycyclic Aromat. Compd. 1996, 8, 213-227. doi:10.1080/10406639608048349

104.Liotta, D.; Saindane, M.; Barnum, C.; Zima, G. Tetrahedron 1985, 41 4881-4889. doi:10.1016/S0040-4020(01)96727-4

105. Gilchrist, T. L.; Summersell, R. J. J. Chem. Soc., Perkin Trans. 1 1988, 2595-2601. doi:10.1039/P19880002595

106. Yang, C.-W.; Liu, R.-S. Tetrahedron Lett. 2007, 48, 5887-5889 doi:10.1016/j.tetlet.2007.06.045

107.Lian, J.-J.; Lin, C.-C.; Chang, H.-K.; Chen, P.-C.; Liu, R.-S. J. Am. Chem. Soc. 2006, 128, 9661-9667. doi:10.1021/ja061203b

108. Mori, N.; Ikeda, S.-i.; Sato, Y. J. Am. Chem. Soc. 1999, 121, 2722-2727. doi:10.1021/ja983348r

109. Ikeda, S.-i.; Kondo, H.; Mori, N. Chem. Commun. 2000, 815-816. doi:10.1039/b001151g

110.Sambaiah, T.; Li, L.-P.; Huang, D.-J.; Lin, C.-H.; Rayabarapu, D. K.; Cheng, C.-H. J. Org. Chem. 1999, 64, 3663-3670. doi:10.1021/jo9900580

111.Unlu, C. S.; Tutar, A.; Erenler, R. J. Chem. Soc. Pak. 2012, 34, 705-708.

112.Tian, Y.; Uchida, K.; Kurata, H.; Hirao, Y.; Nishiuchi, T.; Kubo, T. J. Am. Chem. Soc. 2014, 136, 12784-12793. doi:10.1021/ja507005c

113. Jones, D. W.; Marmon, R. J. J. Chem. Soc., Perkin Trans. 1 1990, 3271-3275. doi:10.1039/p19900003271

114. Siret, B.; Albrecht, S.; Defoin, A. C. R. Chim. 2014, 17, 1075-1079. doi:10.1016/j.crci.2014.01.024 
115. Ohmori, H.; Maeda, H.; Ueda, C.; Masui, M. J. Chem. Soc., Chem. Commun. 1988, 13, 874-875. doi:10.1039/C39880000874

116.Panasiewicz, M.; Zdrojewski, T.; Chrulski, K.; Wojtasiewicz, A.; Jończyk, A. ARKIVOC 2009, No. vii, 98-110.

117.Fang, Z.; Liu, Y.; Barry, B.-D.; Liao, P.; Bi, X. Org. Lett. 2015, 17, 782-785. doi:10.1021/ol5034332

118.Senaiar, R. S.; Teske, J. A.; Young, D. D.; Deiters, A. J. Org. Chem. 2007, 72, 7801-7804. doi:10.1021/jo7013565

119. Hoye, T. R.; Baire, B.; Niu, D.; Willoughby, P. H.; Woods, B. P. Nature 2012, 490, 208-212. doi:10.1038/nature 11518

120.Woods, B. P.; Baire, B.; Hoye, T. R. Org. Lett. 2014, 16, 4578-4581. doi:10.1021/ol502131r

121.Vandyck, K.; Matthys, B.; Van der Eycken, J. Tetrahedron Lett. 2005, 46, 75-78. doi:10.1016/j.tetlet.2004.11.028

122. Hashmi, A. S. K.; Ding, L.; Bats, J. W.; Fisher, P.; Frey, W. Chem. - Eur. J. 2003, 9, 4339-4345. doi:10.1002/chem.200305092

123. Ciabattoni, J.; Crowley, J. E.; Kende, A. S. J. Am. Chem. Soc. 1967, 89, 2778-2779. doi:10.1021/ja00987a074

124. Mostert, S.; Petzer, A.; Petzer, J. P. ChemMedChem 2015, 10 , 862-873. doi:10.1002/cmdc.201500059

125. Fadare, O. A.; Akinpelu, D. A.; Ejemubu, H.; Obafemi, C. A. Afr. J. Pure Appl. Chem. 2014, 8, 68-77. doi:10.5897/AJPAC2014.0558

126. Clark, W. M.; Kassick, A. J.; Plotkin, M. A.; Eldridge, A. M.; Lantos, I. Org. Lett. 1999, 1, 1839-1842. doi:10.1021/ol991111+

127.Yu, D.; Huangfu, G.; Yang, Y. Synthetic method of 2-((1-benzyl-4-piperidyl)-hydroxy-methyl)-5, 6-dimethoxy-1-indanone. Chinese Patent CN102,516,156 A, June 27, 2012.

128.Långvik, O.; Sandberg, T.; Wärnå, J.; Murzin, D. Y.; Leino, R. Catal. Sci. Technol. 2015, 5, 150-160. doi:10.1039/C4CY01099J

129.Zhang, Q.; Chen, C.; Ma, H.; Miao, H.; Zhang, W.; Sun, Z.; Xu, J. J. Chem. Technol. Biotechnol. 2008, 83, 1364-1369. doi:10.1002/jctb.1977

130.Zhang, P.; Lu, H.; Zhou, Y.; Zhang, L.; Wu, Z.; Yang, S.; Shi, H.; Zhu, Q.; Chen, Y.; Dai, S. Nat. Commun. 2015, 6, No. 8446. doi:10.1038/ncomms9446

131.Lv, X.-M.; Kong, L.-J.; Lin, Q.; Liu, X.-F.; Zhou, Y.-M.; Jia, E. Synth. Commun. 2011, 41, 3215-3222. doi:10.1080/00397911.2010.517611

132. Harvey, R. G.; Young, R. J.; Cortez, C.; Lee, H.; Luna, E. J. Org. Chem. 1993, 58, 361-365. doi:10.1021/jo00054a018

133.Lee, H.; Harvey, R. G. J. Org. Chem. 1988, 53, 4253-4256. doi:10.1021/jo00253a017

134.Zhang, J.-L.; Huang, J.-S.; Che, C.-M. Chem. - Eur. J. 2006, 12, 3020-3031. doi:10.1002/chem.200501510

135.Zeng, X.-M.; Chen, J.-M.; Middleton, K.; Zhdankin, V. V. Tetrahedron Lett. 2011, 52, 5652-5655. doi:10.1016/j.tetlet.2011.08.097

136.Jaouen, G.; Meyer, A. J. Am. Chem. Soc. 1975, 97, 4667-4672. doi:10.1021/ja00849a032

137. Asachenko, A. F.; Izmer, V. V.; Babkin, A. V.; Beletskaya, I. P.; Voskoboynikov, A. Z. Russ. Chem. Bull. 2008, 57, 2564-2571. doi:10.1007/s11172-008-0369-0

\section{License and Terms}

This is an Open Access article under the terms of the Creative Commons Attribution License

(http://creativecommons.org/licenses/by/4.0), which permits unrestricted use, distribution, and reproduction in any medium, provided the original work is properly cited.

The license is subject to the Beilstein Journal of Organic Chemistry terms and conditions:

(http://www.beilstein-journals.org/bjoc)

The definitive version of this article is the electronic one which can be found at:

doi: $10.3762 /$ bjoc. 13.48 